\title{
Drivers of i-DNA formation in a variety of environments revealed by four-dimensional UV melting and annealing
}

\author{
Mingpan Cheng*, Jielin Chen, Huangxian Ju, Jun Zhou*, Jean-Louis Mergny* \\ * Correspondence may be sent to either M. Cheng (m821315457@outlook.com), or J. Zhou (jun.zhou@nju.edu.cn), \\ or J.-L. Mergny (jean-louis.mergny@inserm.fr).
}

\section{Table of contents}

Figure S1 SE-HPLC profiles of i-DNAs formed by 20 model sequences (Page S2);

Figure S2 Formation diagram data (Pages S3-S7);

Figure S3 pH dependent $T_{1 / 2}$ (Pages S8-S9);

Figure S4 Temperature dependent $p H_{T}$ (Pages S10-S11);

Figures S5-8 Thermodynamic analysis (Pages S12-S15);

Figure S9 Effects of ionic strength and cation type on the thermal stabilities of 44L1 and 44L3 (Page S16);

Figure S10 Effects of molecular crowding agents on the thermal stabilities of 44L1 and 44L3 (Page S17);

Figure S11 Summary of ionic strength, cation type and molecular crowding agents on the thermal stabilities of 44L1 and 44L3 (Page S18);

Figure $\mathbf{S 1 2} \mathrm{pH}$ dependent CD spectra of 20 model sequences (Page S19);

Figure S13 CD spectra of promoter i-DNAs at pH 5.0 or 8.0 (Page S20);

Figure S14 SE-HPLC profiles of i-DNAs in human gene promoters (Page S21);

Figure S15 TDS of promoter i-DNAs at different pHs and temperatures (Pages S22-S23);

Figure S16 Formation fraction of promoter i-DNAs at different pHs and temperatures (Pages S24-S25);

Figure S17 Formation diagram of promoter i-DNAs at different pHs and temperatures (Pages S26-S29);

Figure S18 UV-melting and annealing of promoter i-DNAs under pH 7.0 with crowding agents (Page S30);

Figure S19 Standard Gibbs free energy $\left(\Delta G^{\circ}\right)$ at $20^{\circ} \mathrm{C}$ of promoter i-DNAs (Page S31);

Table S1 Raw data of $\mathrm{pH}$ dependent $T_{1 / 2}$ of 20 model sequences (Pages S32-S38);

Table S2 Linear fitting results of $T_{1 / 2}$ as a function of $\mathrm{pH}$, and $p H_{T}$ as a function of temperature for all 25 sequences (Page S39);

Table S3 Raw data of temperature dependent $\mathrm{pH}_{T}$ of 20 model sequences (Pages S40-S44);

Table S4 Linear fitting results of standard $\Delta G^{\circ}$ as a function of $\mathrm{pH}$ (Page S45);

Table S5 Raw data pH dependent $T_{1 / 2}$ of promoter i-DNAs (Pages S46-S50);

Table S6 Raw data temperature dependent $\mathrm{pH}_{T}$ of promoter i-DNAs (Pages S51-S55);

Table S7 $T_{1 / 2}$ of promoter i-DNAs obtained from UV melting and annealing processes under crowding environments (Page S56);

Table S8 Thermodynamics of promoter i-DNAs without or with crowding agents (Page S57). 
Figure S1 Aggregation state demonstrated by SE-HPLC at pH 6.0.
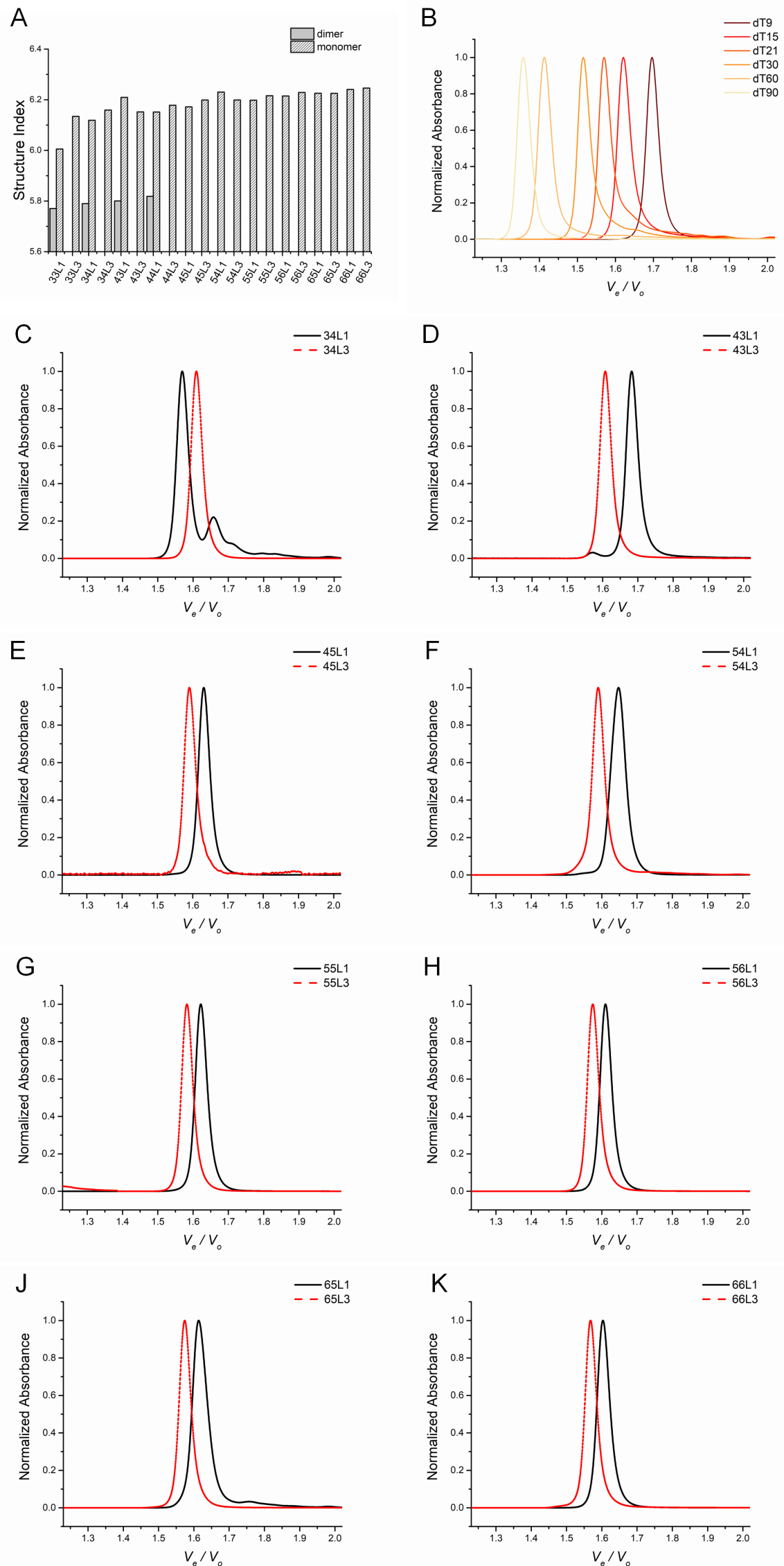

Figure S1 Aggregation state demonstrated by SE-HPLC at pH 6.0. (A) Structure index for each i-motif deduced from the $V_{e} / V_{o}$ for each peak and strand molecular weight. Normalized chromatograms of (B) dT ladders: dT9, dT15, dT21, dT30, dT60, dT90, (C) 34L1, 34L3, (D) 43L1, 43L3, (E) 45L1, 45L3, (F) 54L1, 54L3, (G) 55L1, 55L3, (H) 56L1, 56L3, (J) 65L1, 65L3, (K) 66L1, 66L3. 
Figure S2 Formation diagrams of i-motifs: 33L1, 33L3, 34L1, 34L3.
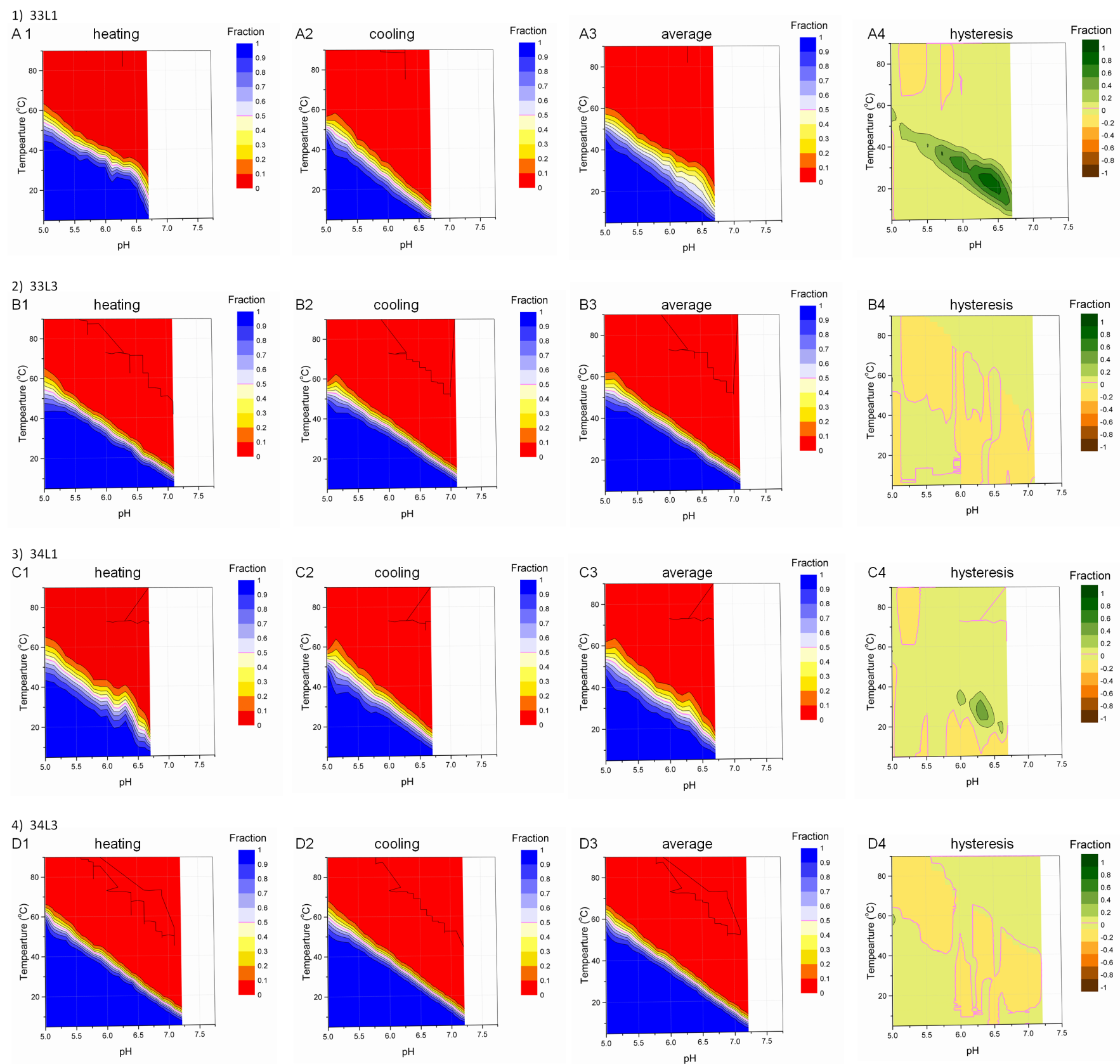

Figure S2 Formation diagrams of i-motifs deduced from the heating process (left column), the cooling process (second column), average of the heating and cooling processes (third column), and difference between heating and cooling processes (hysteresis, right column). (A) 33L1, (B) 33L3, (C) 34L1, (D) 34L3. 
Figure S2 Formation diagrams of i-motifs: 43L1, 43L3, 44L1, 44L3. (continued_01)
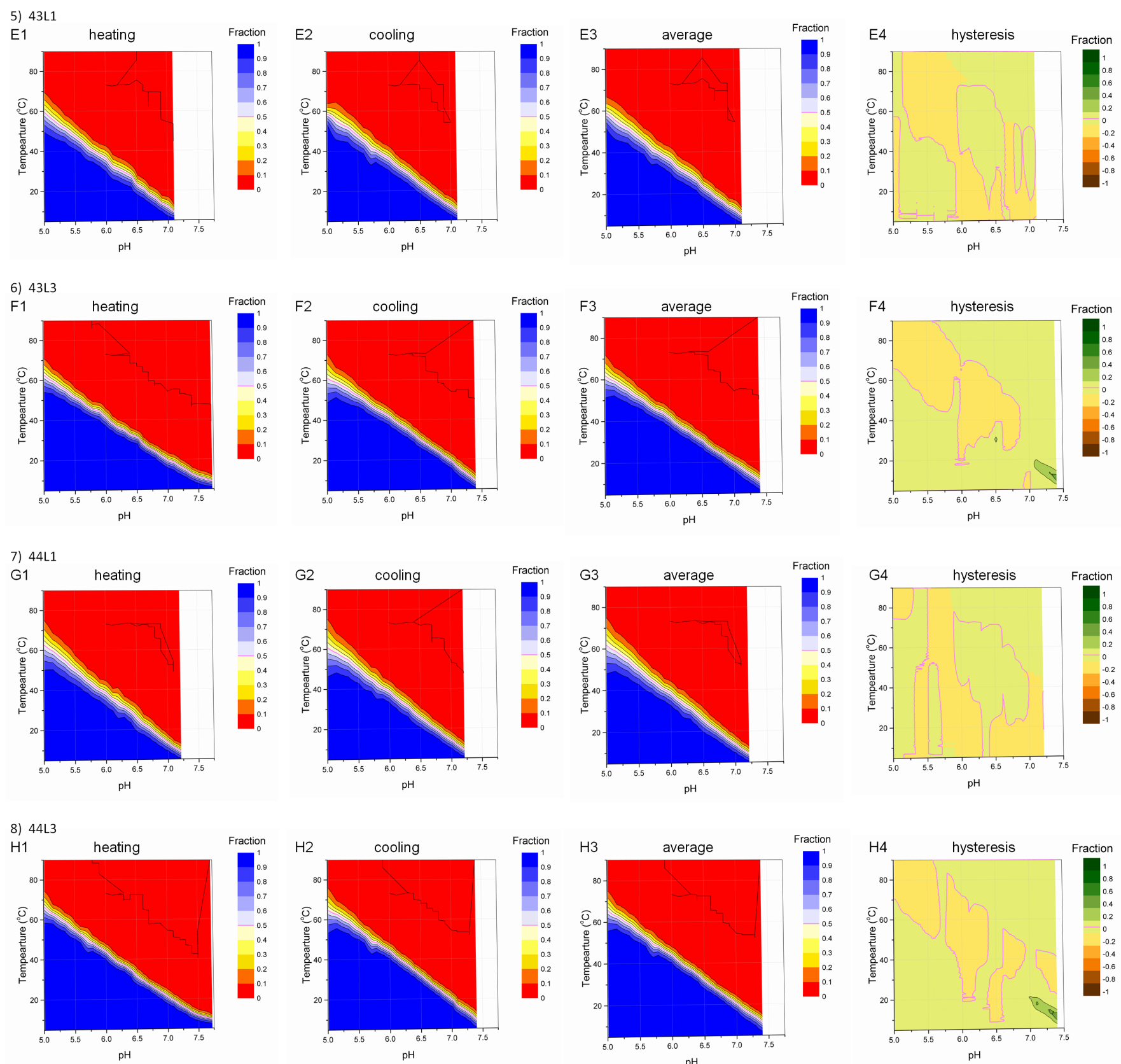

Figure S2, continued. Formation diagrams of i-motifs deduced from the heating process (left column), the cooling process (second column), average of the heating and cooling processes (third column), and difference between heating and cooling processes (hysteresis, right column). (E) 43L1, (F) 43L3, (G) 44L1, (H) 44L3. 
Figure S2 Formation diagrams of i-motifs: 45L1, 45L3, 54L1, 54L3. (continued_02)
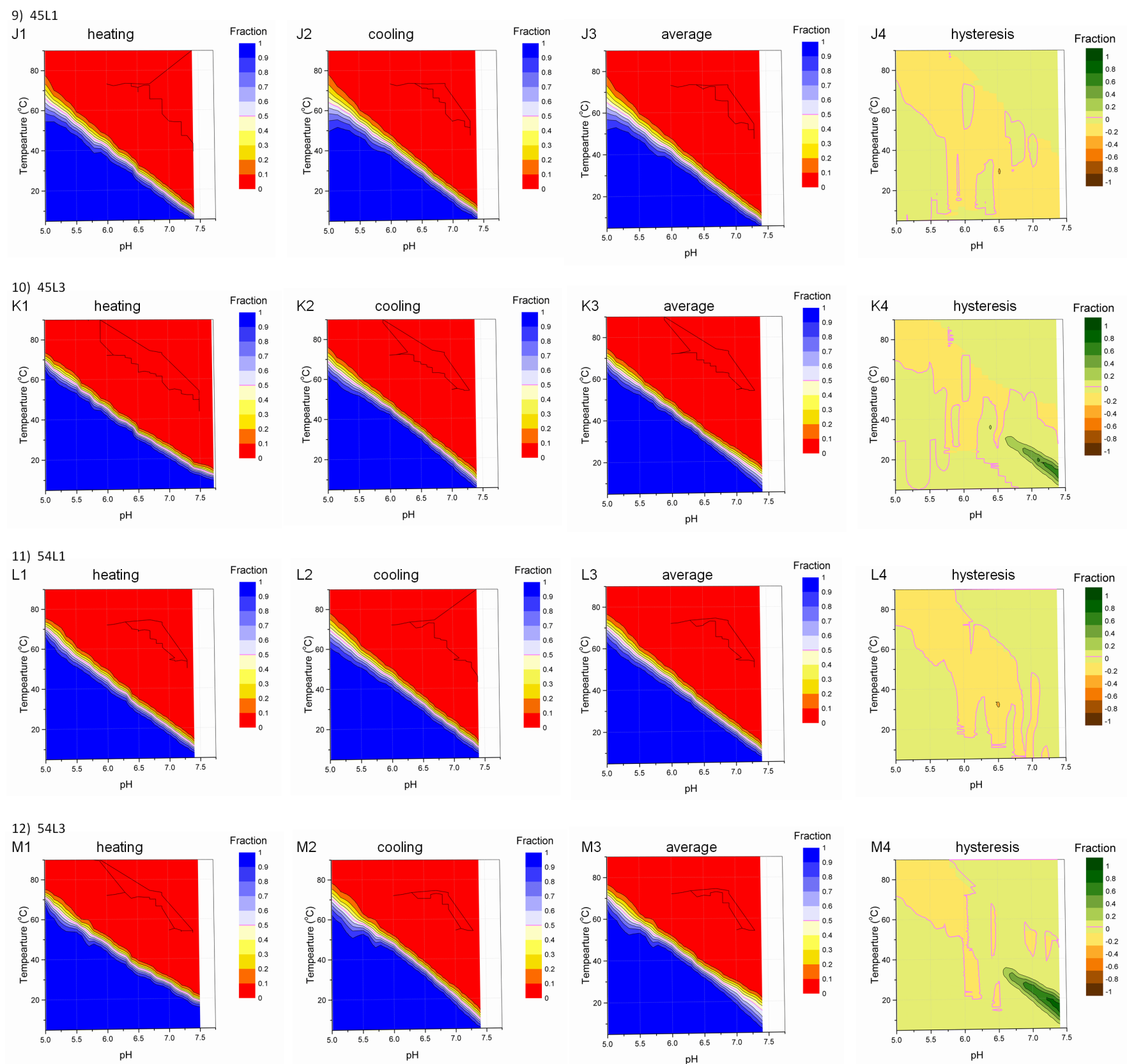

Figure S2 continued. Formation diagrams of i-motifs deduced from the heating process (left column), the cooling process (second column), average of the heating and cooling processes (third column), and difference between heating and cooling processes (hysteresis, right column). (J) 45L1, (K) 45L3, (L) 54L1, (M) 54L3. 
Figure S2 Formation diagrams of i-motifs: 55L1, 55L3, 56L1, 56L3. (continued_03)
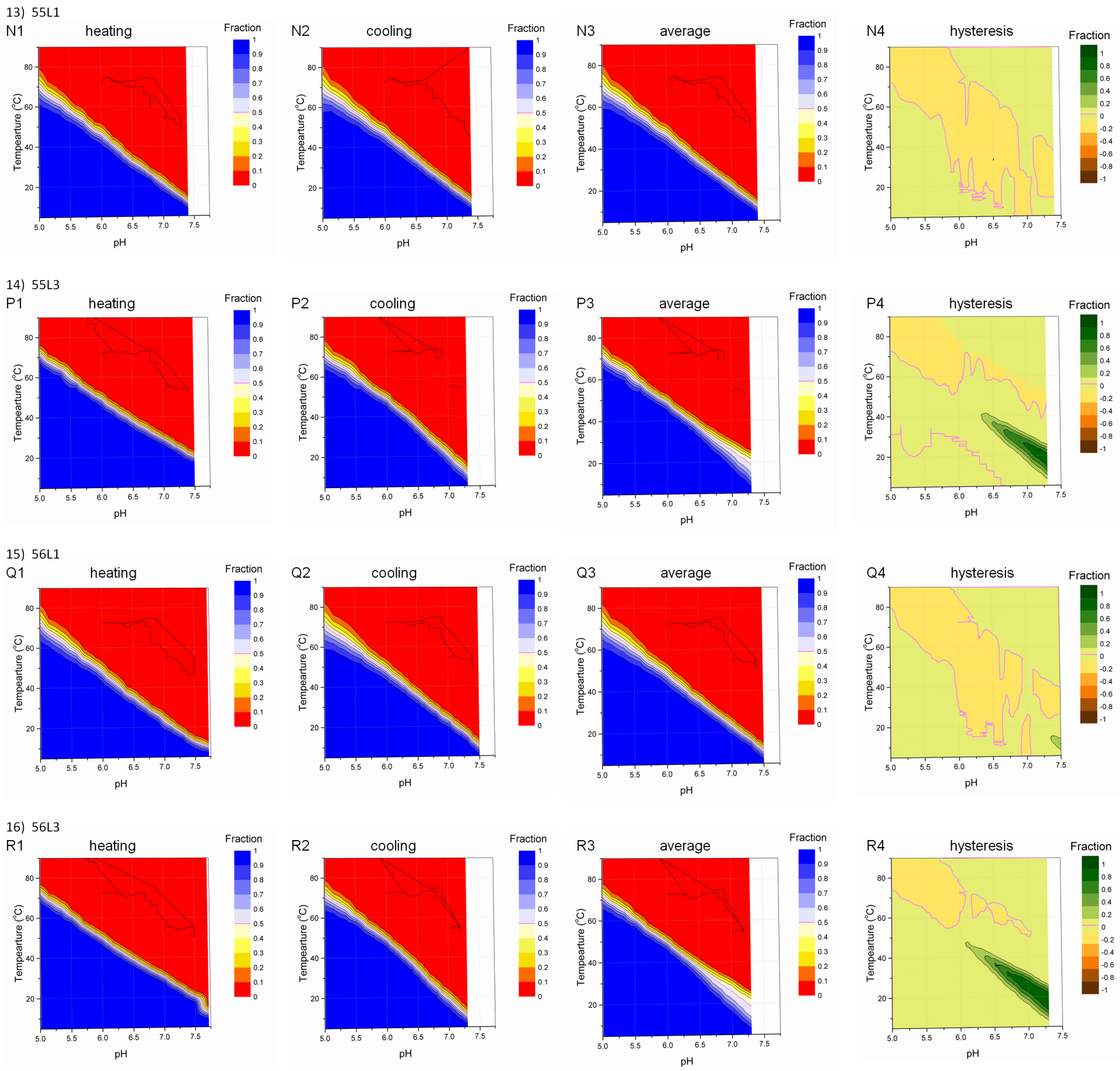

Figure S2 continued. Formation diagrams of i-motifs deduced from the heating process (left column), the cooling process (second column), average of the heating and cooling processes (third column), and difference between heating and cooling processes (hysteresis, right column). (N) 55L1, (P) 55L3, (Q) 56L1, (R) 54L3. 
Figure S2 Formation diagrams of i-motifs: 65L1, 65L3, 66L1, 66L3. (continued_04)
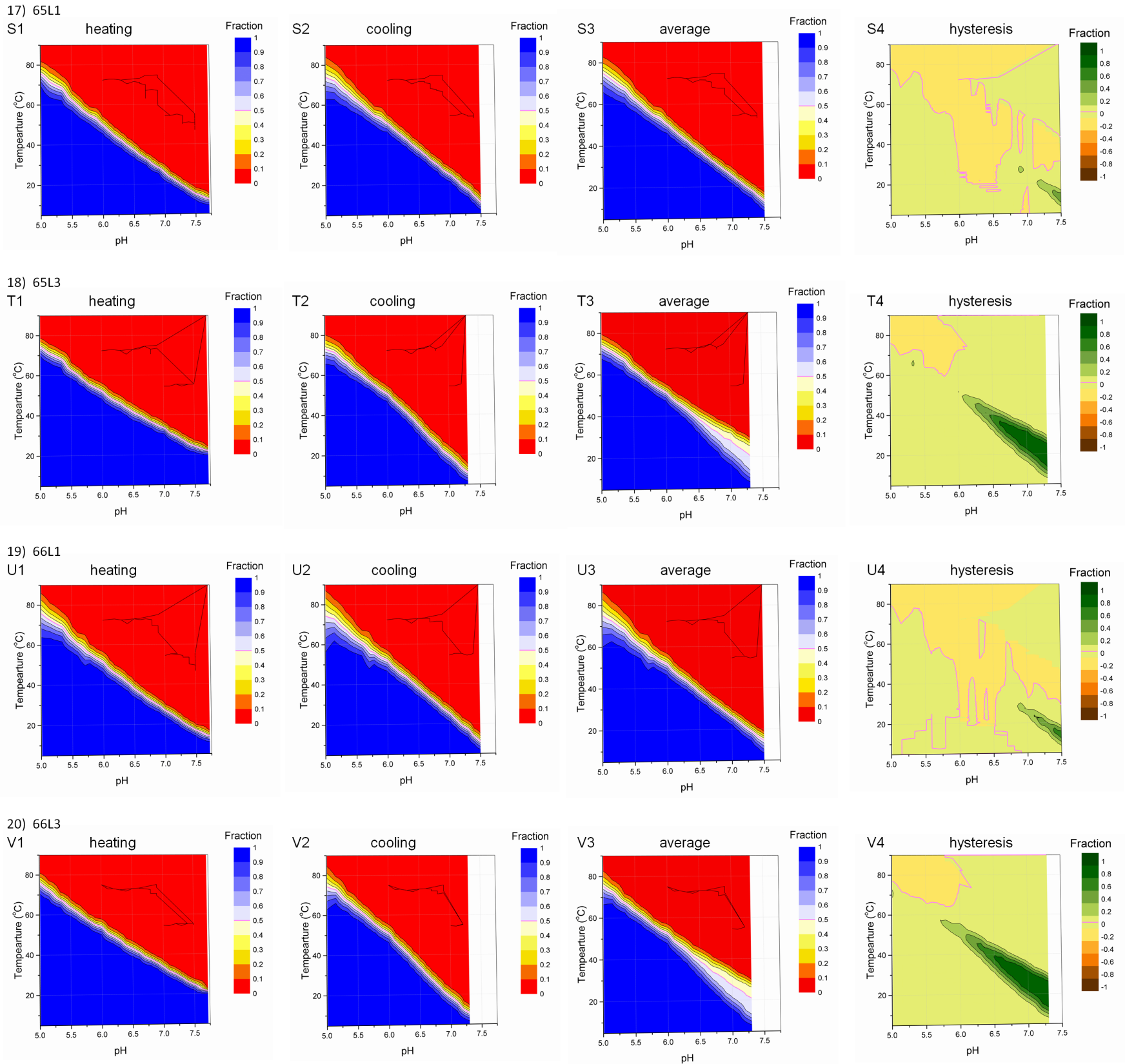

Figure S2 continued. Formation diagrams of i-motifs deduced from the heating process (left column), the cooling process (second column), average of the heating and cooling processes (third column), and difference between heating and cooling processes (hysteresis, right column). (S) 65L1, (T) 65L3, (U) 66L1, (V) 66L3. 
Figure S3 $T_{1 / 2}$ obtained from UV melting and annealing processes at different pHs.
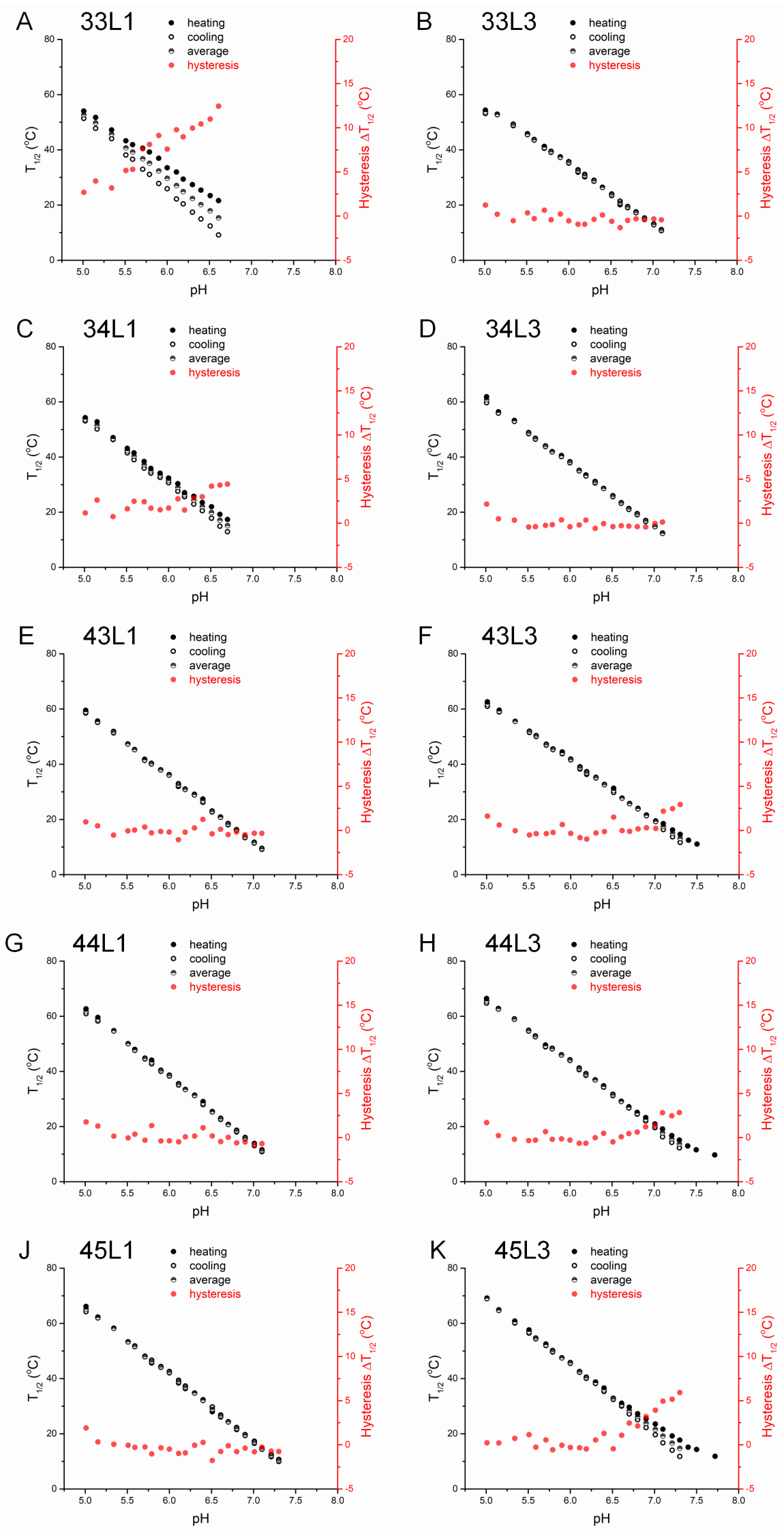

Figure S3 $T_{1 / 2}$ obtained from UV melting and annealing processes at different pH solutions. (A) 33L1, (B) 33L3. (C) 34L1, (D) 34L3, (E) 43L1, (F) 43L3, (G) 44L1, (H) 44L3, (J) 45L1, (K) 45L3. Data are summarized in Table S1. 
Figure S3 $T_{1 / 2}$ obtained from UV melting and annealing processes. (Continued_01)
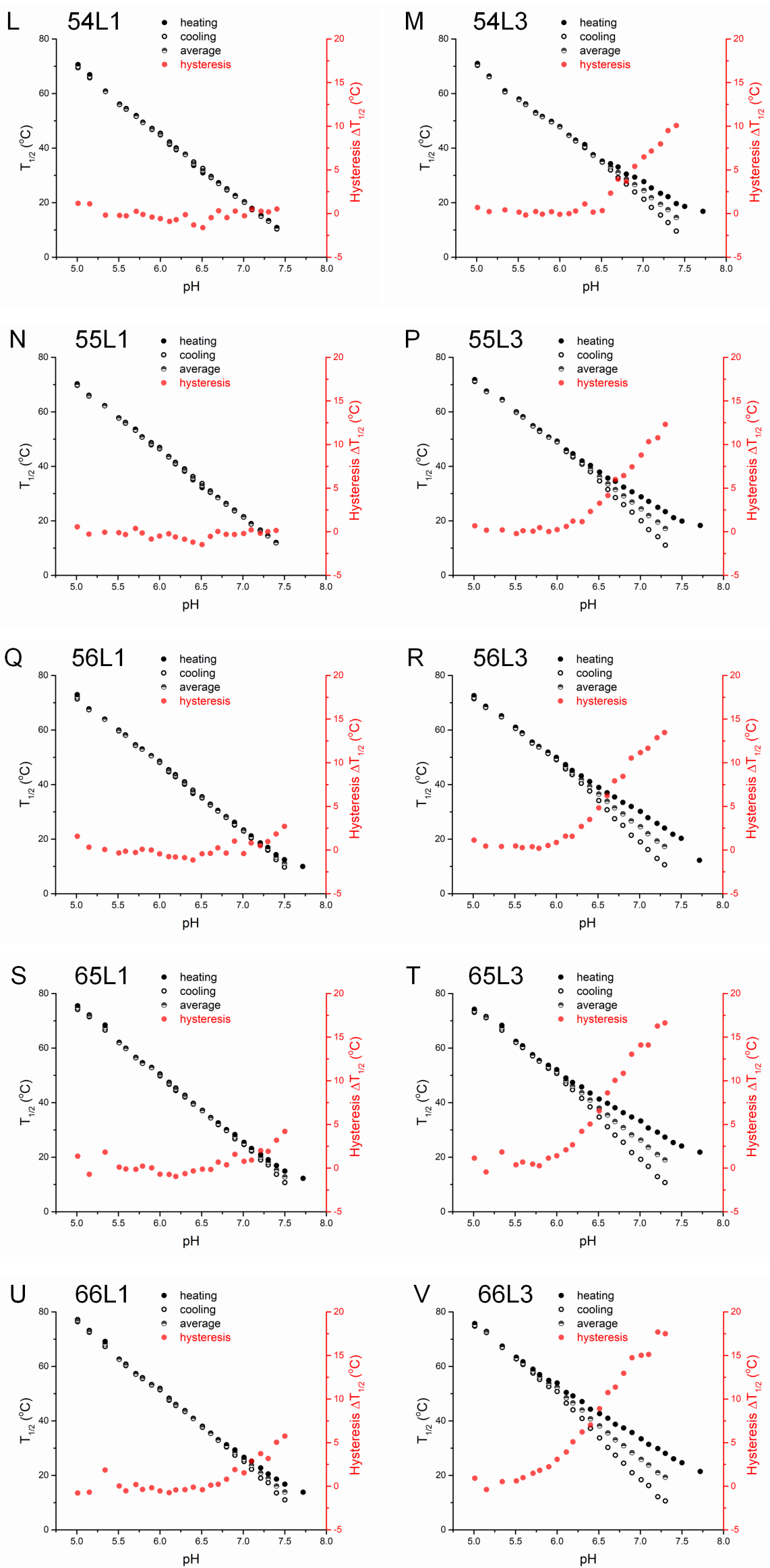

Figure S3 $T_{1 / 2}$ obtained from UV melting and annealing processes at different pHs. (L) 54L1, (M) 54L3, (N) 55L1, (P) 55L3, (Q) 56L1, (R) 56L3, (S) 65L1, (T) 65L3, (U) 66L1, (V) 66L3. Data are summarized in Table S1. 
Figure S4 $\mathrm{pH}_{T}$ obtained from UV melting and annealing processes at different temperatures.
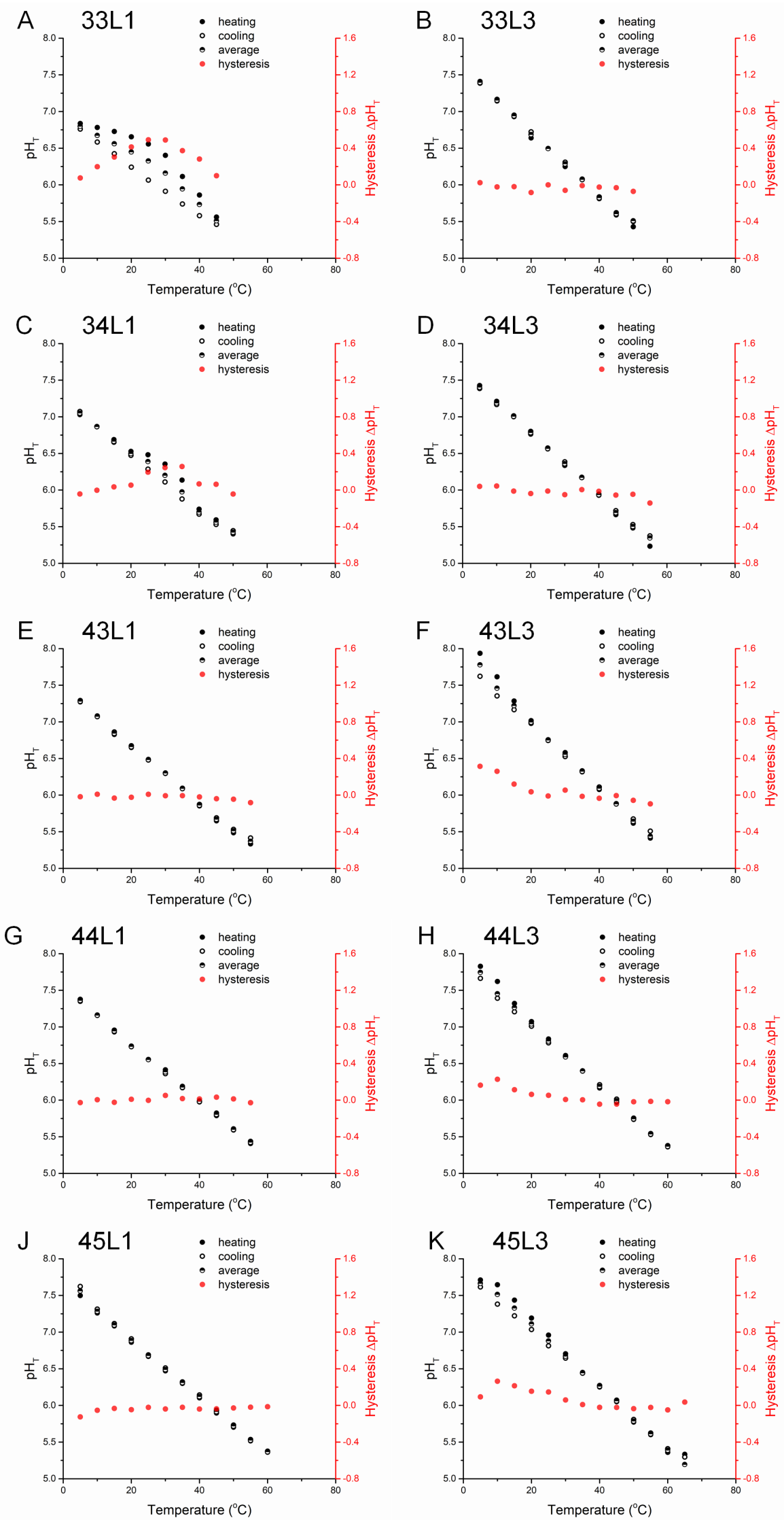

Figure $\mathbf{S 4} \mathrm{pH}_{T}$ obtained from UV melting and annealing processes at different temperatures. (A) 33L1, (B) 33L3. (C) 34L1, (D) 34L3, (E) 43L1, (F) 43L3, (G) 44L1, (H) 44L3, (J) 45L1, (K) 45L3. Data are summarized in Table S3. 
Figure $\mathbf{S} 4 \mathrm{pH}_{\mathrm{T}}$ obtained from UV melting and annealing processes. (Continued_01)
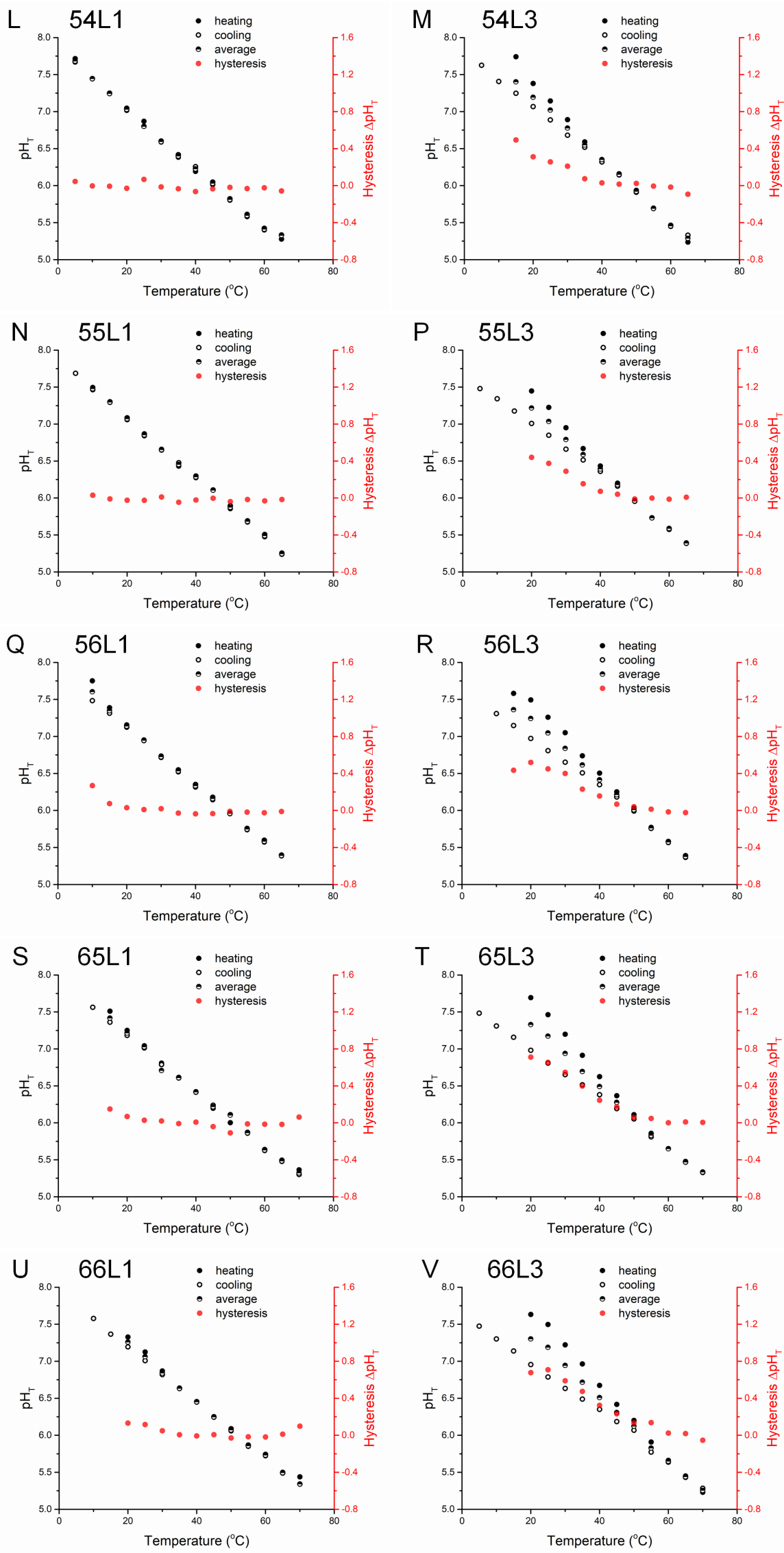

Figure S4 $\mathrm{pH}_{\mathrm{T}}$ obtained from UV melting and annealing processes at different temperatures. (L) 54L1, (M) 54L3, (N) 55L1, (P) 55L3, (Q) 56L1, (R) 56L3, (S) 65L1, (T) 65L3, (U) 66L1, (V) 66L3. Data are summarized in Table S3. 
Figures S5-8 Thermodynamic analysis of 33L3, 34L3, 43L3 and 44L3.

A

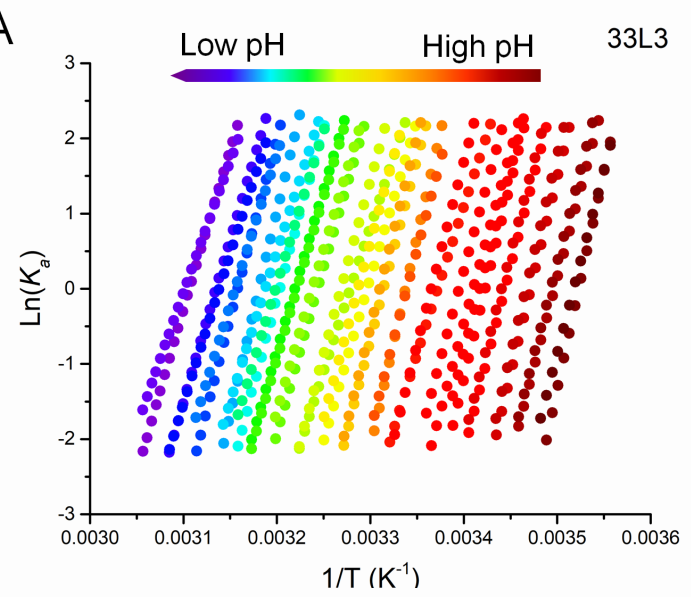

C

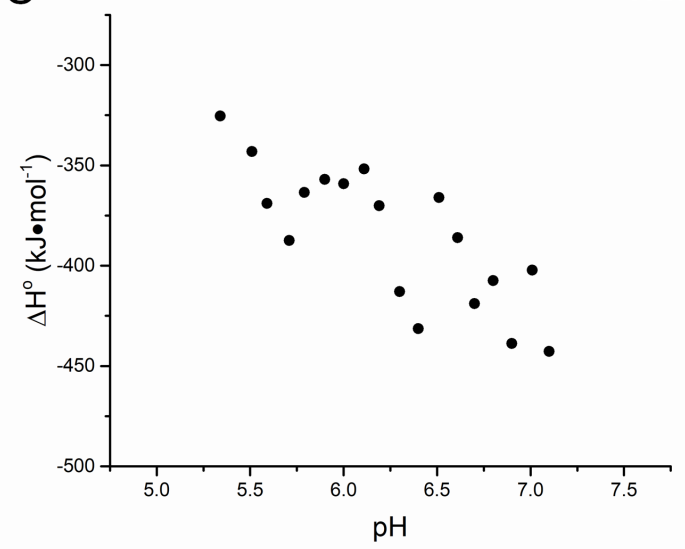

B

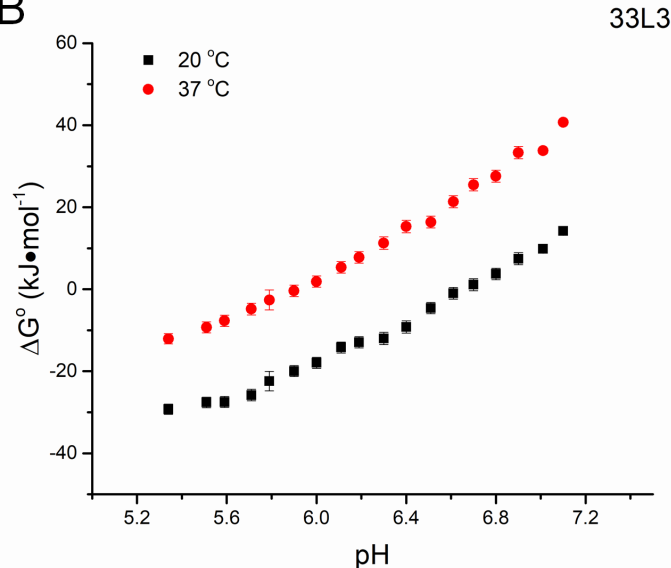

$\mathrm{D}$

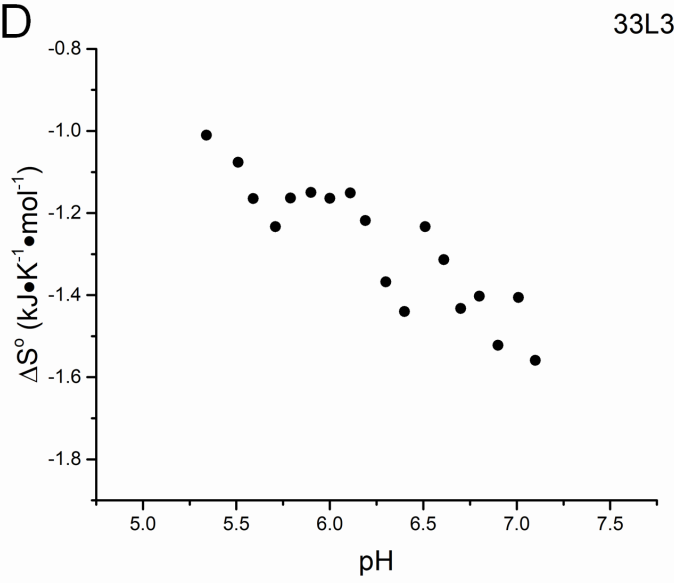

Figure S5 Thermodynamic analysis of $33 \mathrm{~L} 3$ at varied $\mathrm{pH}$. (A) van't Hoff plot: $\mathrm{Ln}\left(K_{a}\right)$ as function of $1 / \mathrm{T} . K_{a}$ is the equilibrium constant between folding and unfolding, $T$ is the temperature in Kelvins; (B) Gibbs free energy change $\Delta G^{\circ}$ at $20^{\circ} \mathrm{C}$ and $37^{\circ} \mathrm{C}$; (C) Standard enthalpy change $\Delta H^{\circ}$; (D) Standard entropy change $\Delta S^{\circ}$. 
A

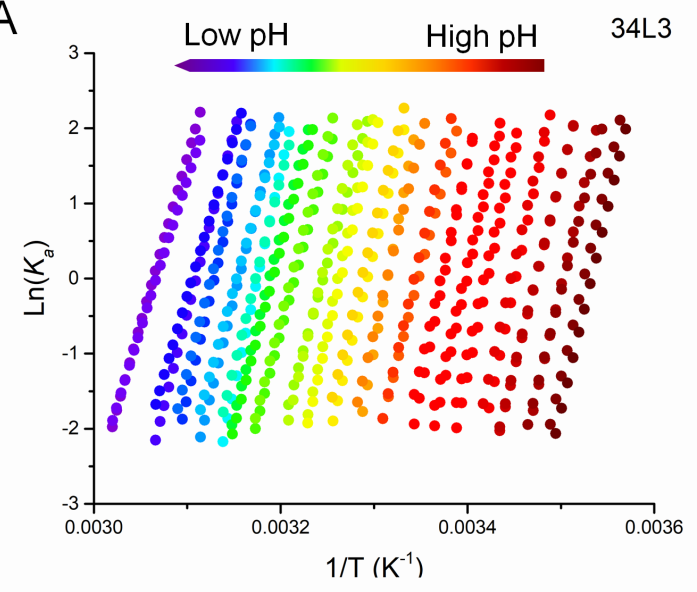

C

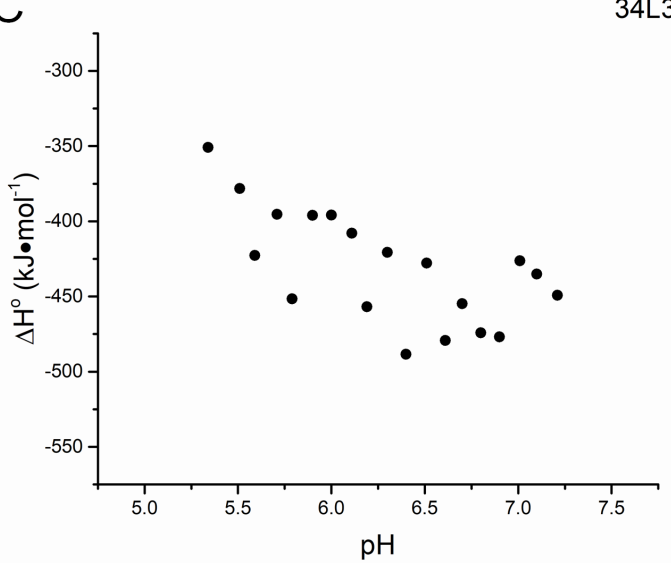

$\mathrm{B}$

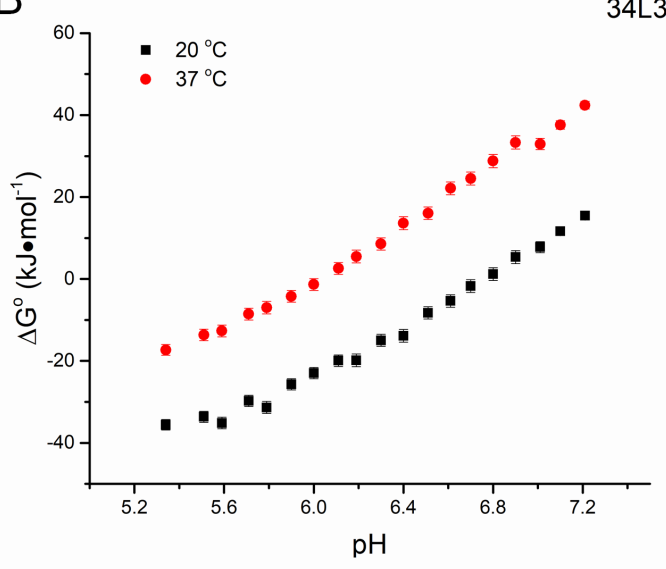

D

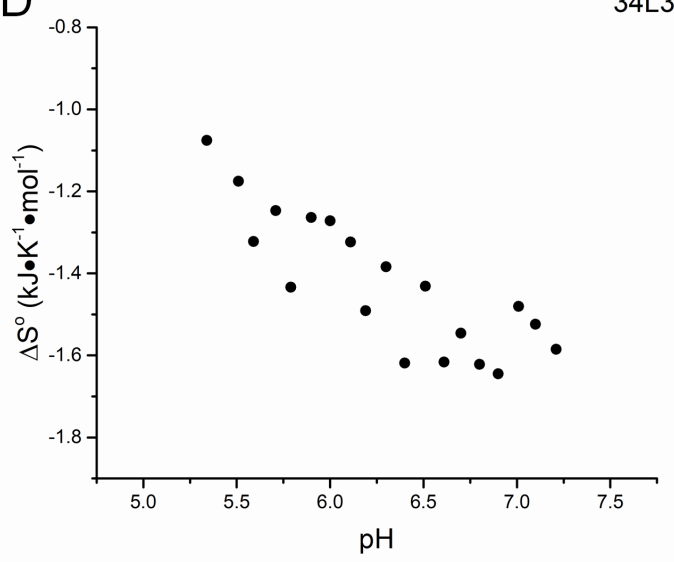

Figure S6 Thermodynamic analysis of $34 \mathrm{~L} 3$ at varied $\mathrm{pH}$. (A) van't Hoff plot: $\mathrm{Ln}\left(K_{a}\right)$ as function of 1/T. $K_{a}$ is the equilibrium constant between folding and unfolding, $T$ is the temperature in Kelvins; (B) Gibbs free energy change $\Delta G^{\circ}$ at $20^{\circ} \mathrm{C}$ and $37^{\circ} \mathrm{C}$; (C) Standard enthalpy change $\Delta H^{\circ}$; (D) Standard entropy change $\Delta S^{\circ}$. 
A

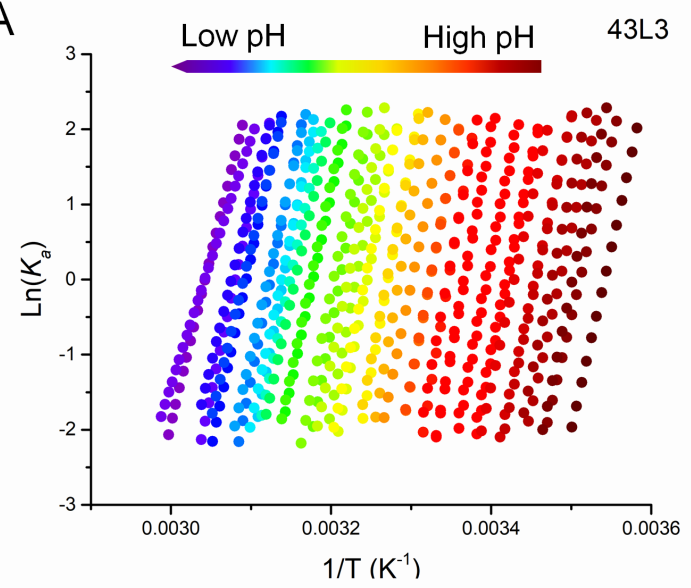

C

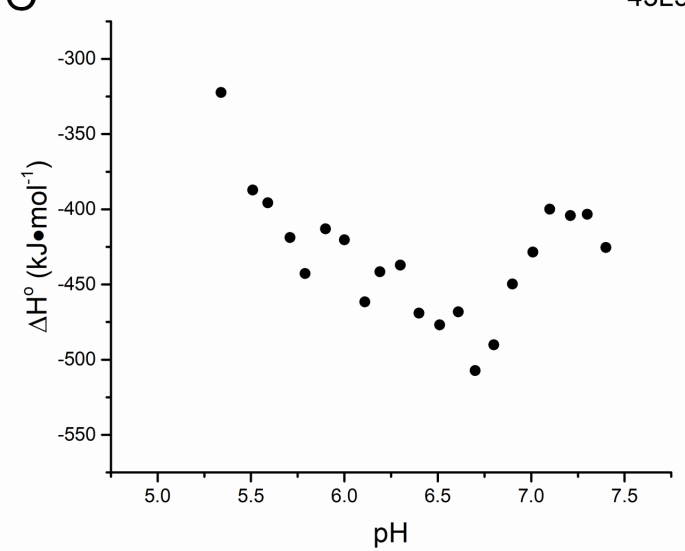

$\mathrm{B}$

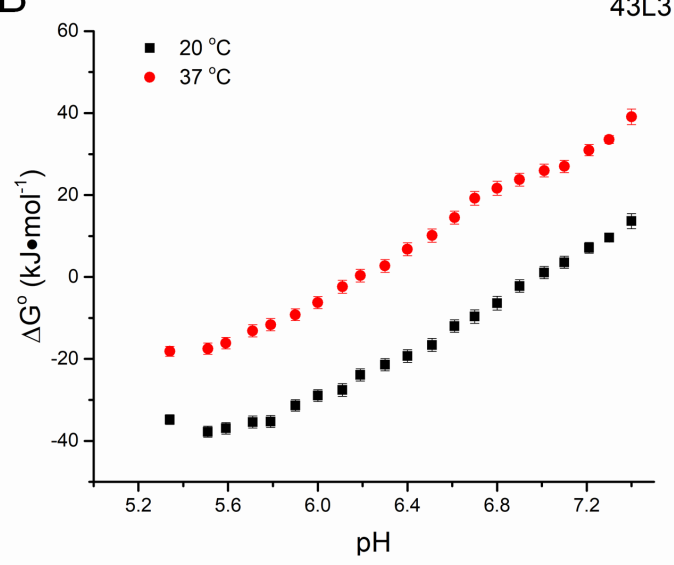

D

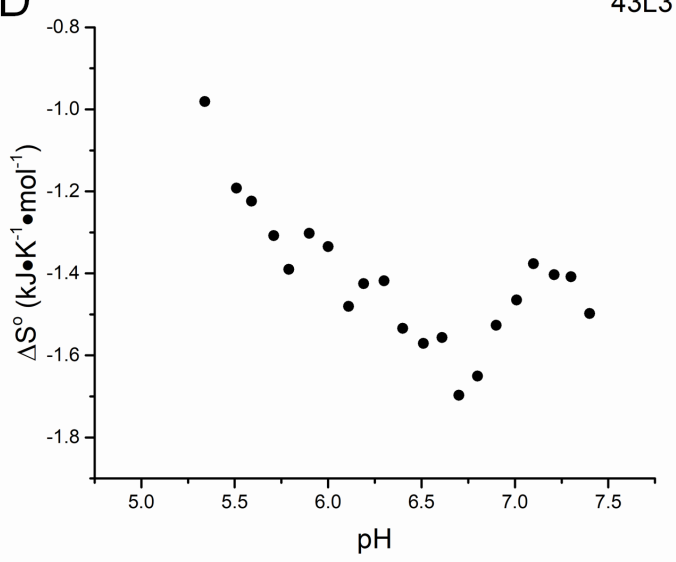

Figure S7 Thermodynamic analysis of $43 \mathrm{~L} 3$ at different $\mathrm{pH}$. (A) van't Hoff plot: $\operatorname{Ln}\left(K_{a}\right)$ as function of $1 / \mathrm{T}$. $K_{a}$ is the equilibrium constant between folding and unfolding, $T$ is the temperature in Kelvins; (B) Gibbs free energy change $\Delta G^{\circ}$ at $20^{\circ} \mathrm{C}$ and $37^{\circ} \mathrm{C}$; (C) Standard enthalpy change $\Delta H^{\circ}$; (D) Standard entropy change $\Delta S^{\circ}$. 

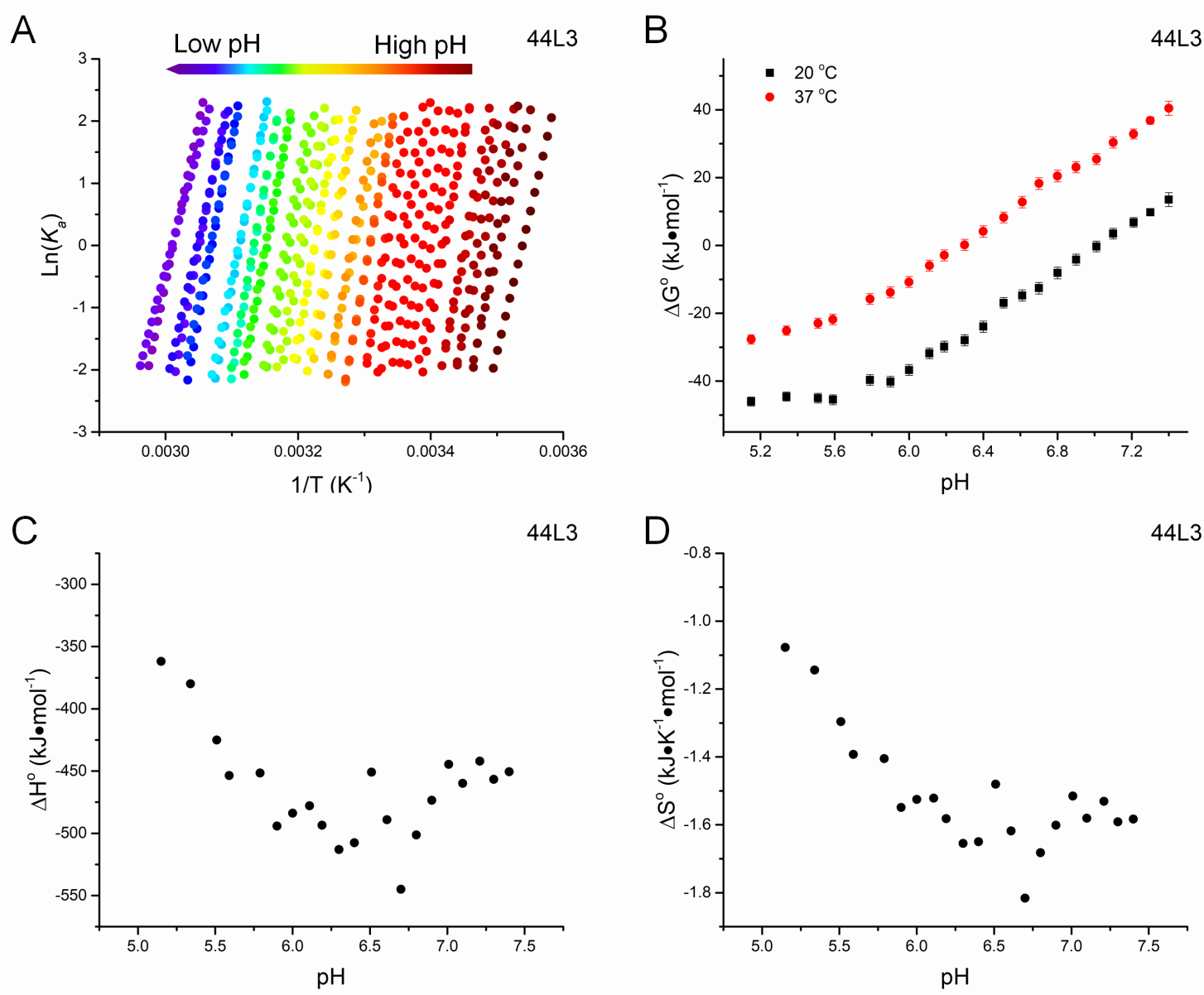

Figure S8 Thermodynamic analysis of $44 \mathrm{~L} 3$ at different $\mathrm{pH}$. (A) van't Hoff plot: $\operatorname{Ln}\left(K_{a}\right)$ as function of 1/T. $K_{a}$ is the equilibrium constant between folding and unfolding, $T$ is the temperature in Kelvins; (B) Gibbs free energy change $\Delta G^{\circ}$ at $20^{\circ} \mathrm{C}$ and $37^{\circ} \mathrm{C}$; (C) Standard enthalpy change $\Delta H^{\circ}$; (D) Standard entropy change $\Delta S^{\circ}$. 
Figure S9 Effects of ionic strength and cation type on the thermal stabilities of 44L1 and 44L3.

A

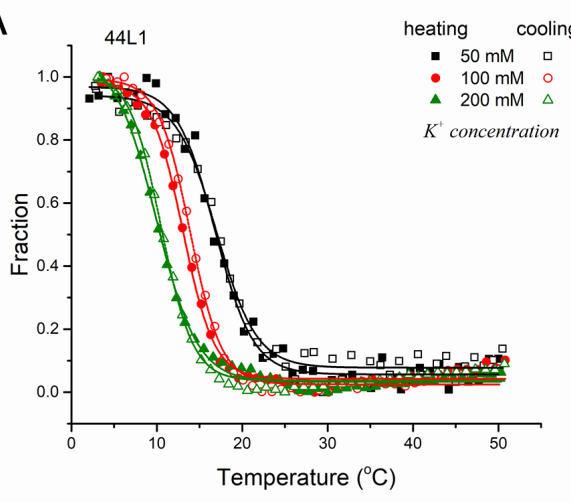

C

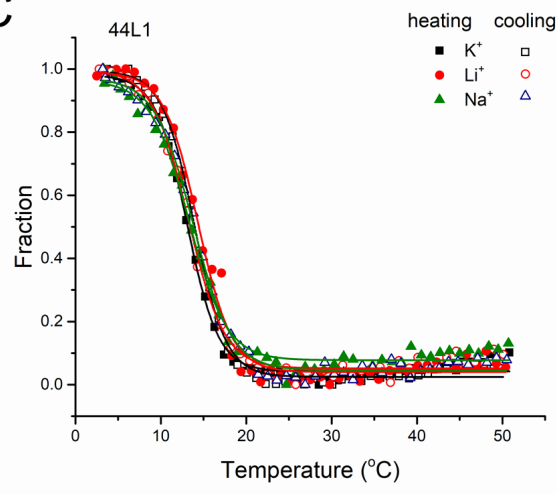

B

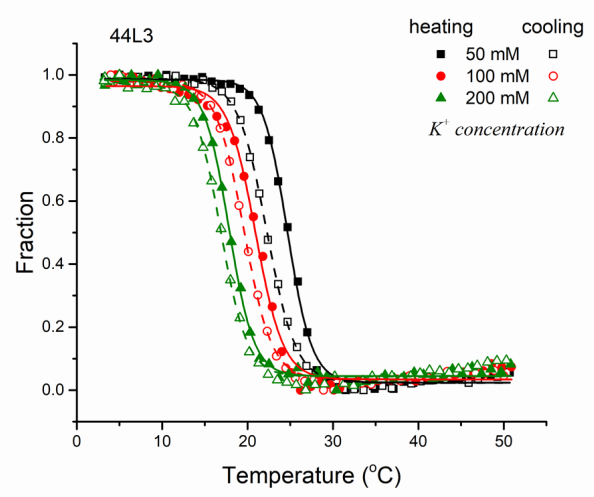

D

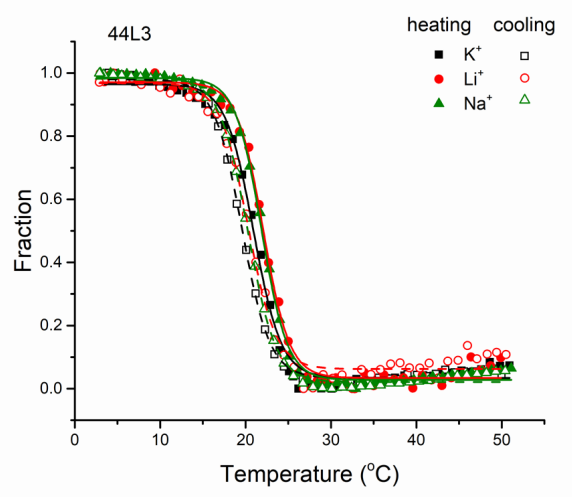

Figure S9 Effects of ionic strength and cation type on thermal stability: (A \& C) 44L1; (B \& D) 44L3. UV melting and annealing profiles at $\mathrm{pH} 7.0$ buffer in the presence of either (A \& B) 50, 100, and $200 \mathrm{mM} \mathrm{K}^{+}$, or (C \& D) $100 \mathrm{mM} \mathrm{K}^{+}, \mathrm{Li}^{+}, \mathrm{Na}^{+}$. 
Figure S10 Effects of molecular crowding agents on the thermal stabilities of 44L1 and 44L3.

A

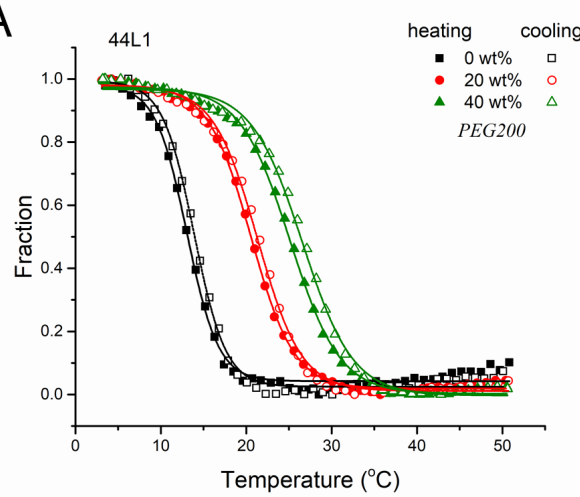

C

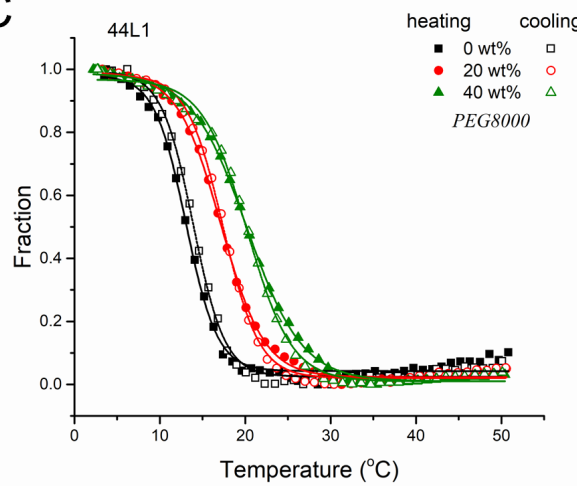

E

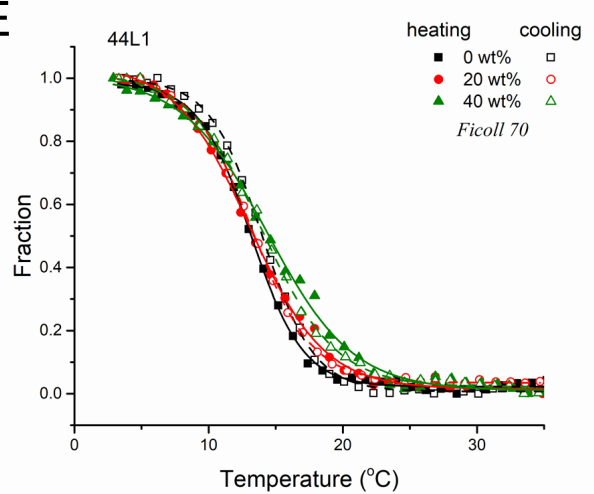

B

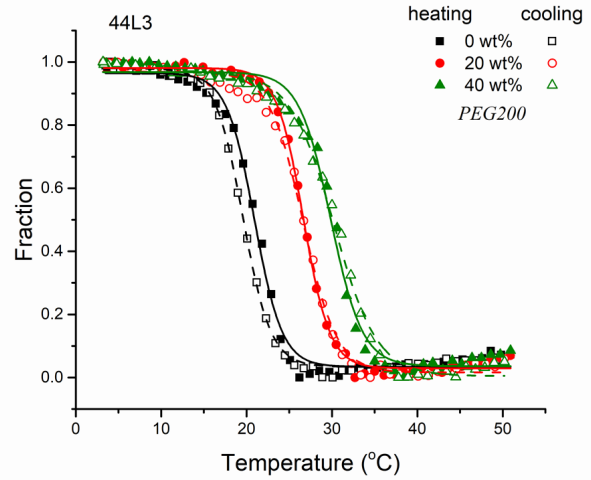

$\mathrm{D}$

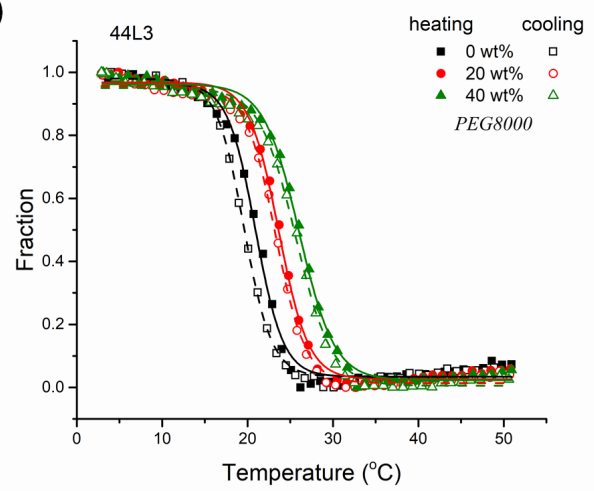

$\mathrm{F}$

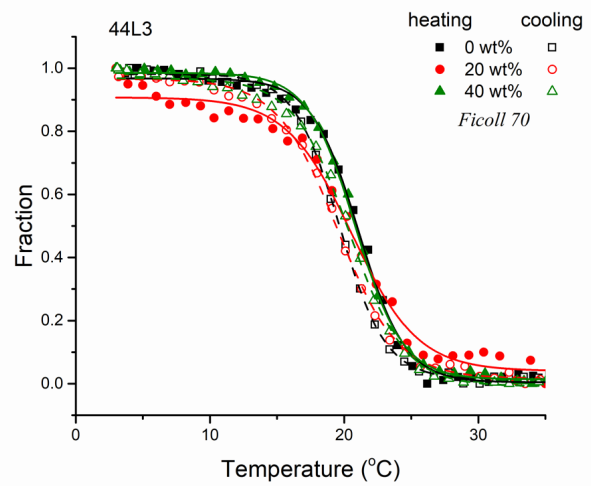

Figure S10 Effects of molecular crowding agents on thermal stability: (A \& C \& D) 44L1; (B \& D \& F) 44L3. UV melting and annealing profiles at pH 7.0 buffer in presence of 0, 20, 40 wt\% (A \& B) PEG200, (C \& D) PEG8000, (E \& F) Ficoll70 and 100 mM K'. 
Figure S11 Summary of ionic strength, cation type and molecular crowding agents on the thermal stabilities of $44 \mathrm{~L} 1$ and $44 \mathrm{~L} 3$.
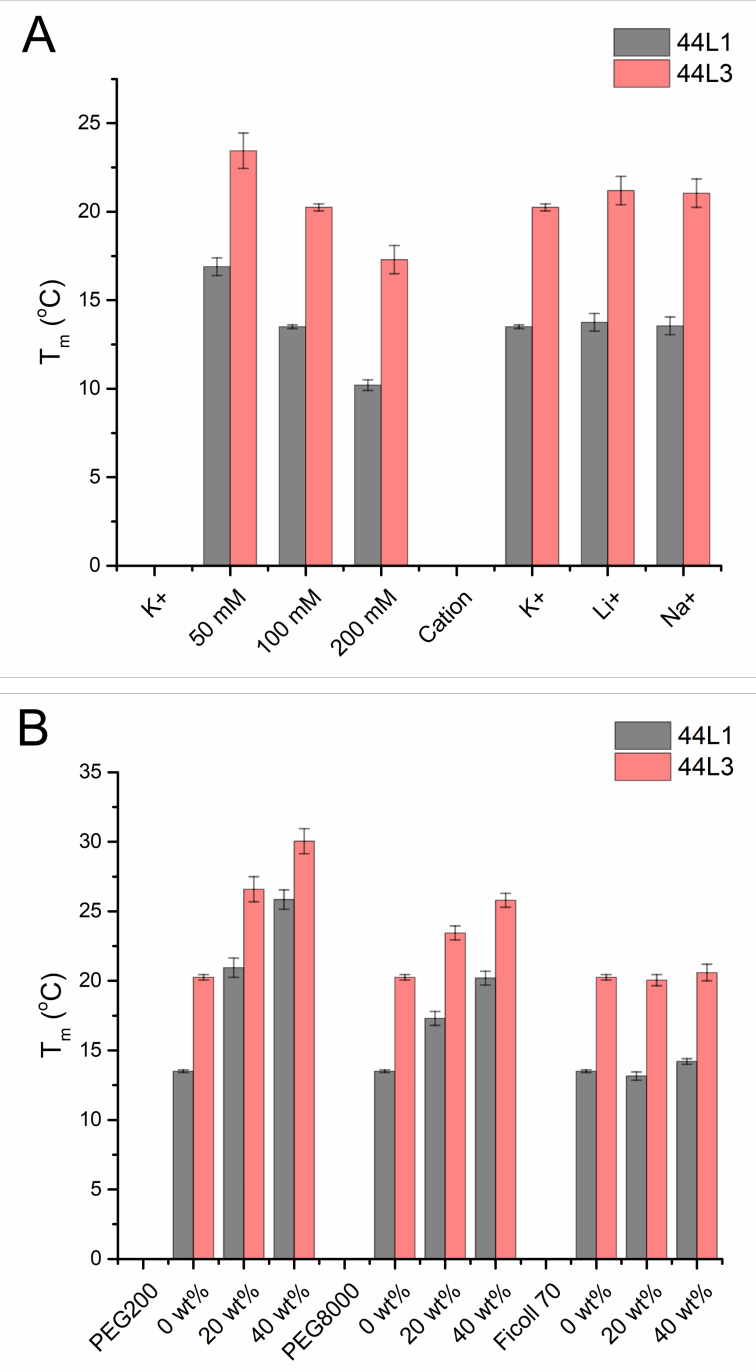

Figure S11 Summary of ionic strength, cation type and molecular crowding agents on the thermal stabilities of $44 \mathrm{~L} 1$ and $44 \mathrm{~L} 3 . T_{m}$ is determined at $\mathrm{pH} 7.0(\mathrm{~A})$ in the presence of 50,100 , or $200 \mathrm{mM} \mathrm{K}^{+}$, or $100 \mathrm{mM} \mathrm{K}^{+}$, $\mathrm{Li}^{+}$and $\mathrm{Na}^{+}$, or (B) in the presence of $100 \mathrm{mM} \mathrm{K}^{+}$with 20 or $40 \mathrm{wt} \%$ PEG200, PEG8000 or Ficoll70. Raw data of UV melting and annealing curves are given in Figures $\mathbf{S 9}$ and $\mathbf{S 1 0}$. (Note: The data in $100 \mathrm{mM} \mathrm{K}^{+}$ were duplicated in panel $\mathrm{A}$ to facilitate comparison). 
Figure S12 pH-dependent CD spectra of 20 model sequences.
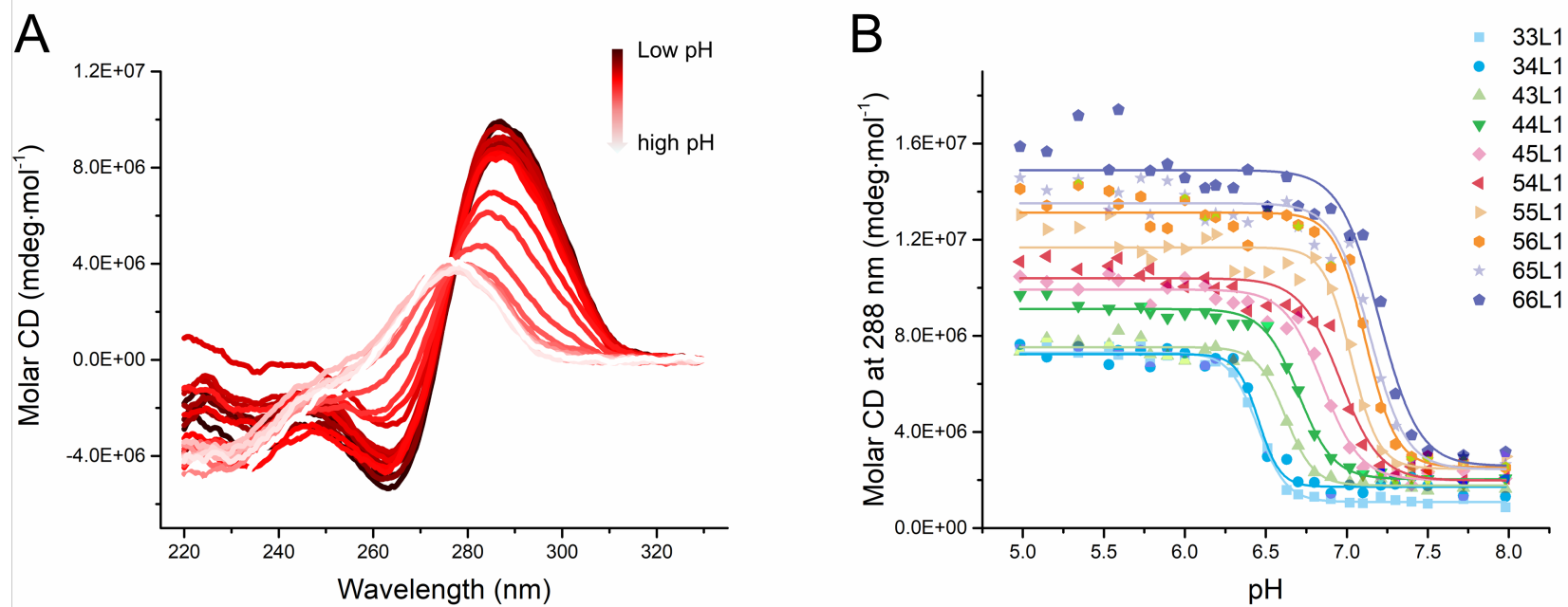

C

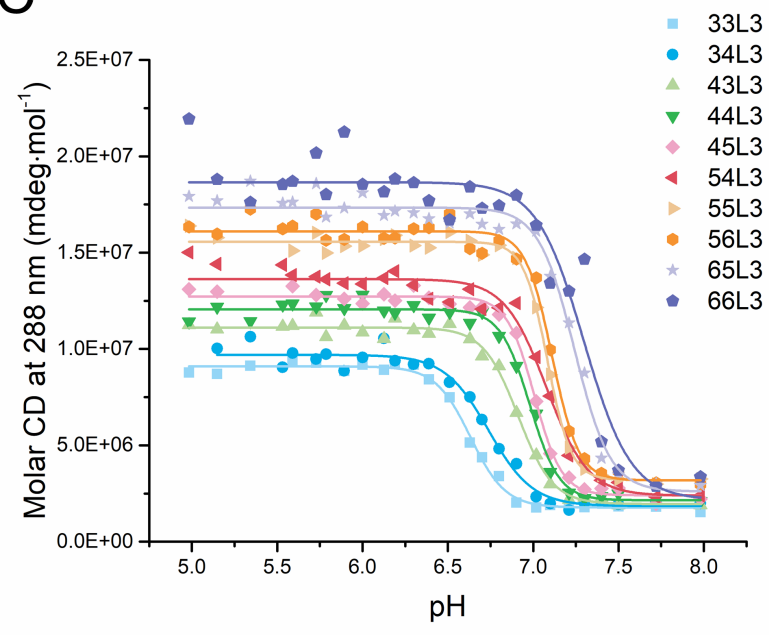

D

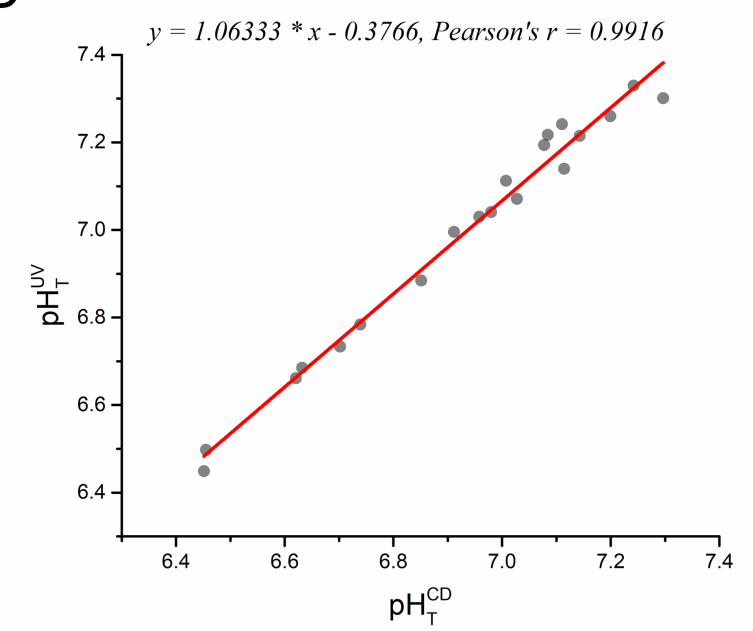

Figure S12 CD spectra at $20{ }^{\circ} \mathrm{C}$. (A) CD spectra of $44 \mathrm{~L} 1$ at various $\mathrm{pH}$ solutions as an example. $\mathrm{pH}$ dependent $\mathrm{CD}$ intensities at $288 \mathrm{~nm}$ as a function of $\mathrm{pH}$ : Sequences with total loop length (B) 3 and (C) 9. (D) $\mathrm{pH}_{T}$ of all 20 sequences at $20{ }^{\circ} \mathrm{C}$ obtained from UV (average data) as a function of that from CD experiments. The same samples were used for both 4DUVMA and CD experiments. 
Figure $\mathbf{S 1 3}$ CD spectra of promoter i-DNAs at pH 5.0 or 8.0 .
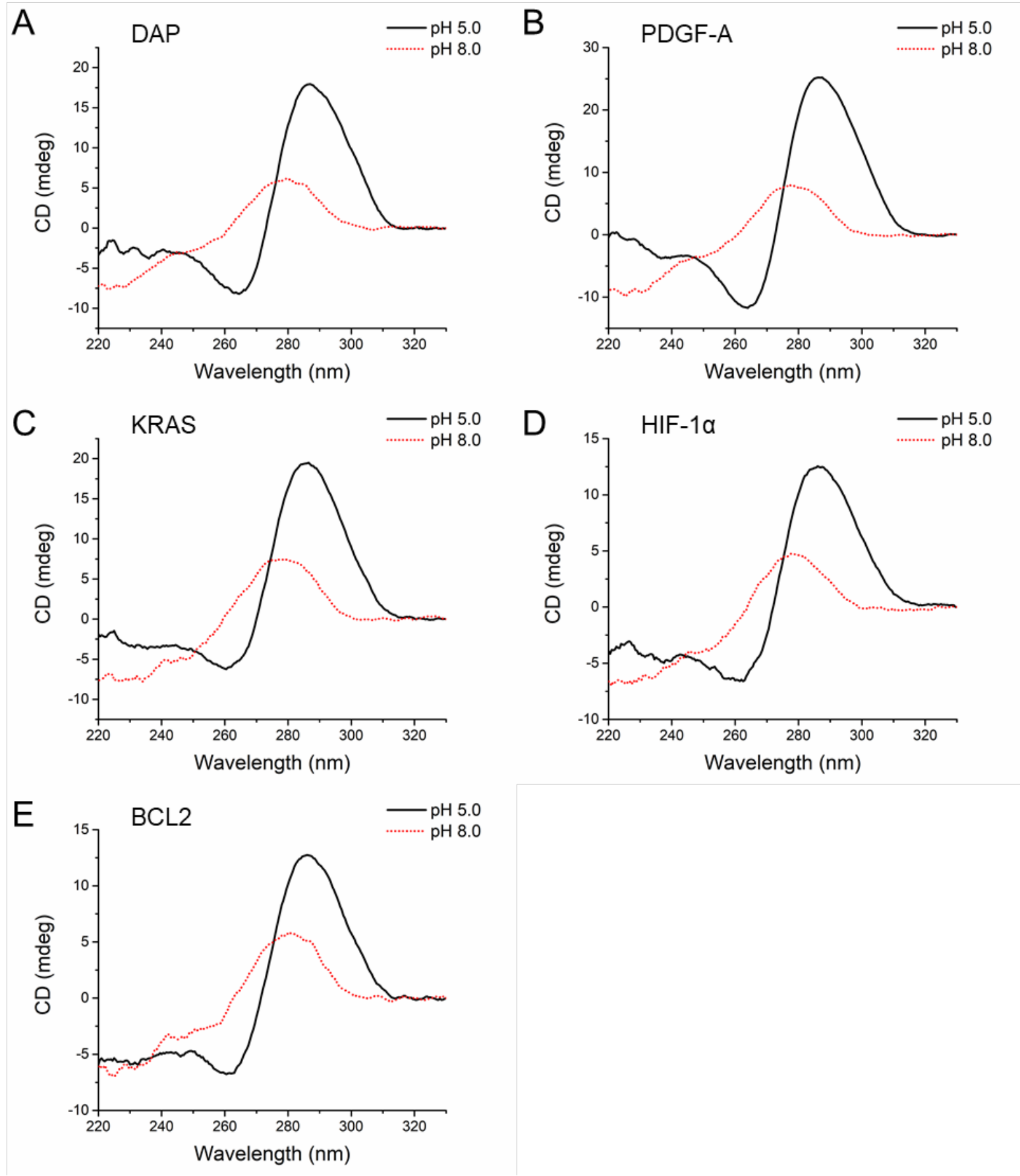

Figure S13 CD spectra of (A) DAP, (B) PDGF-A, (C) KRAS, (D) HIF-1 $\alpha$ and (E) BCL-2 at pH 5.0 or 8.0. 
Figure S14 SE-HPLC profiles of i-DNAs in human gene promoters.

A

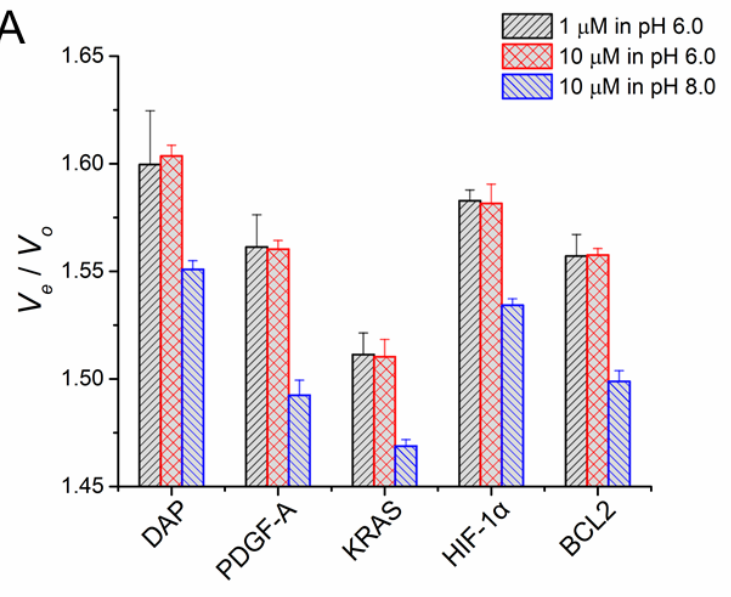

C

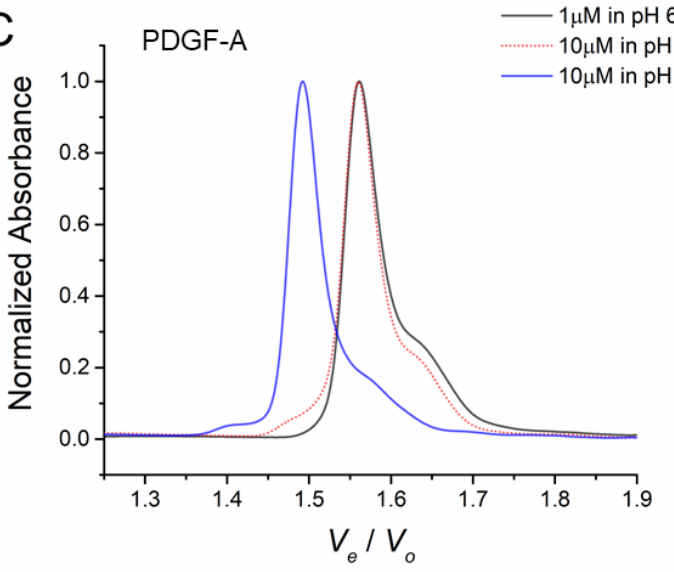

E

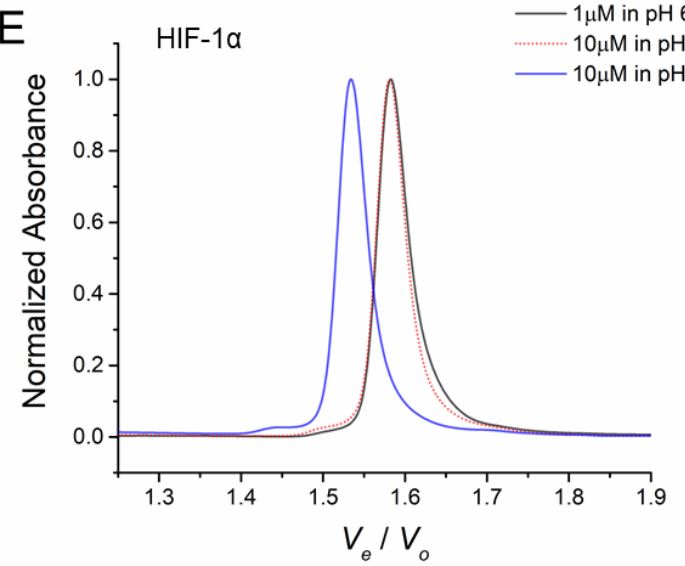

B

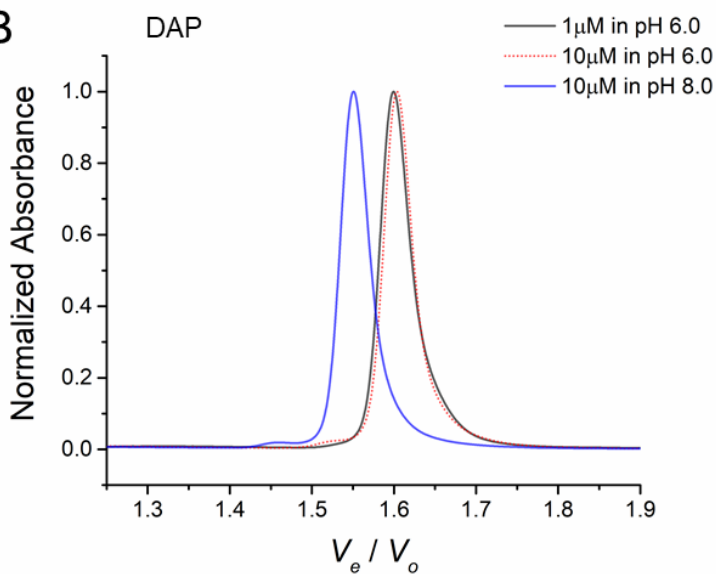

D

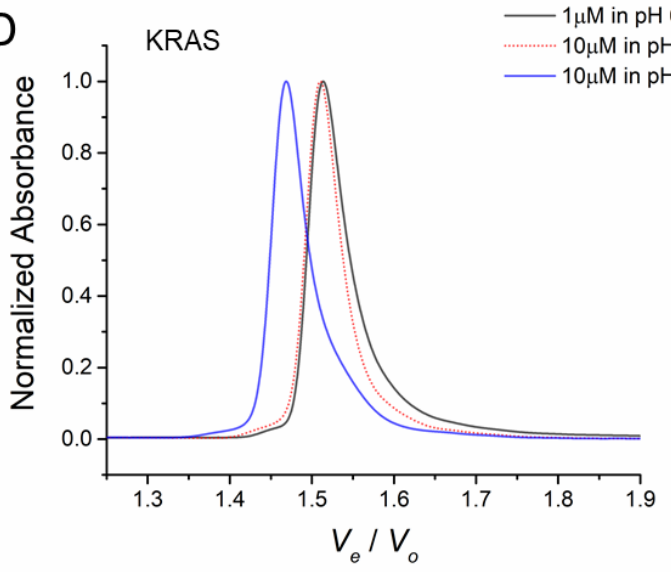

$\mathrm{F}$

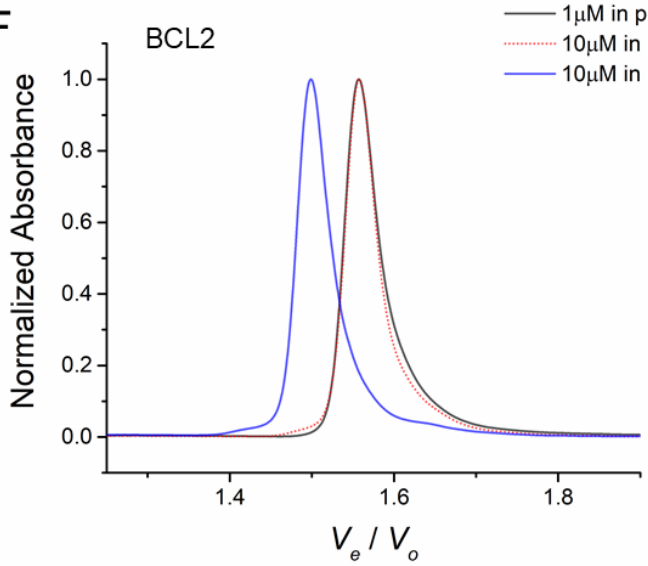

Figure S14 SE-HPLC analysis for the identification of molecularity. (A) Relative elution volume $V_{e} / V_{o}$, where $V_{e}$ is the elution volume and $V_{o}$ is dead volume of the system. Normalized chromatograms of (B) $D A P$, (C) PDGF-A, (D) KRAS, (E) HIF-1 $\alpha$ and (F) BCL-2 under $\mathrm{pH} 6.0$ with 1 and $10 \mu \mathrm{M}$ and under $\mathrm{pH} 8.0$ with $10 \mu \mathrm{M}$. 
Figure S15 TDS of promoter i-DNAs at different pHs and temperatures.
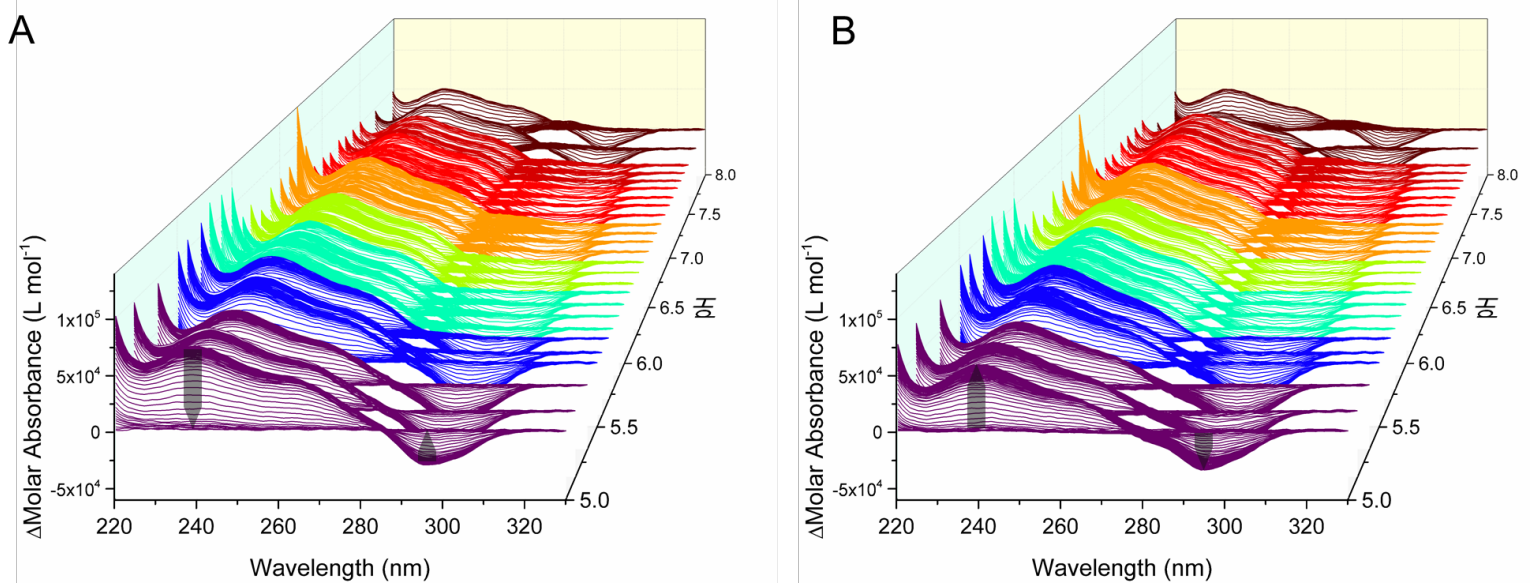

Figure S15.1 The $(\mathbf{A})$ heating and $(\mathbf{B})$ cooling 4DUVMA processes of DAP: molar absorbance difference, wavelength $(320-220 \mathrm{~nm})$, temperature $\left(5.0-95.0^{\circ} \mathrm{C}\right)$ and $\mathrm{pH}(5.0-8.0)$. The directions of temperature changes (from low to high, and high to low) are denoted with grey arrows in the TDS at pH 5.0.
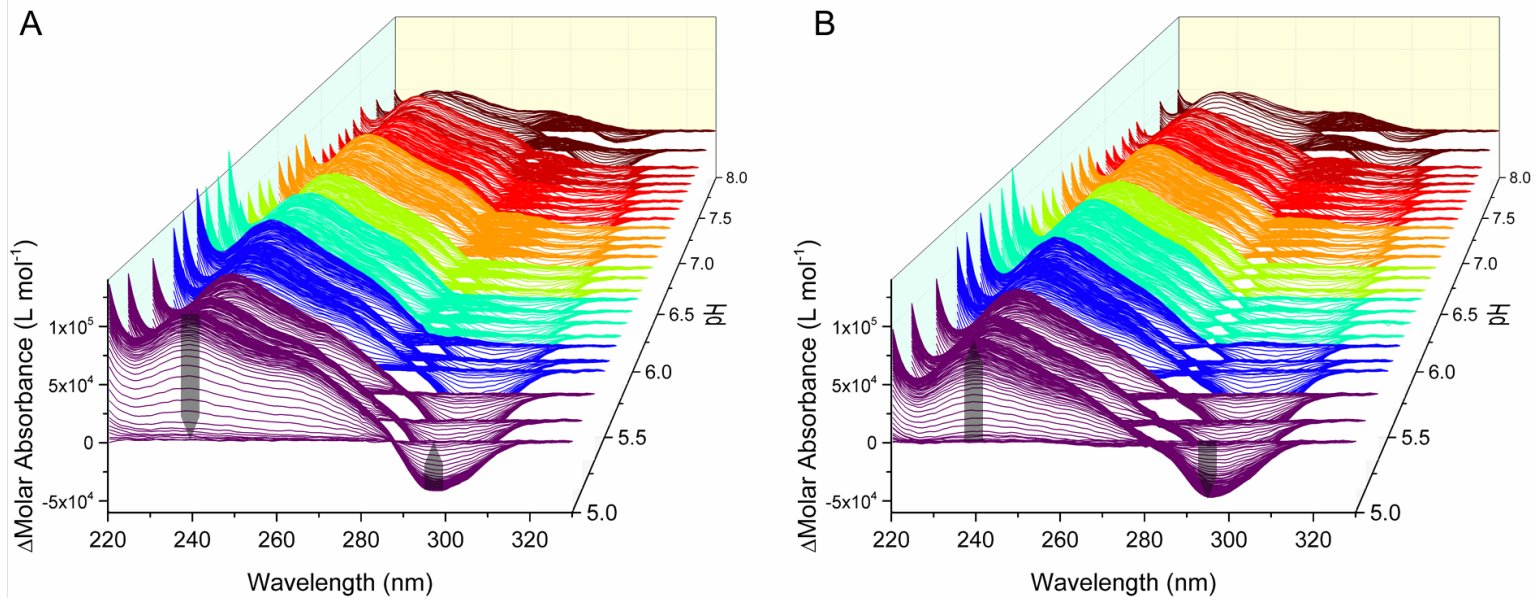

Figure S15.2 The (A) heating and (B) cooling 4DUVMA processes of PDGF-A: molar absorbance difference, wavelength $(320-220 \mathrm{~nm})$, temperature $\left(5.0-95.0{ }^{\circ} \mathrm{C}\right)$ and $\mathrm{pH}(5.0-8.0)$. The directions of temperature changes (from low to high, and high to low) are denoted with grey arrows in the TDS at pH 5.0. 

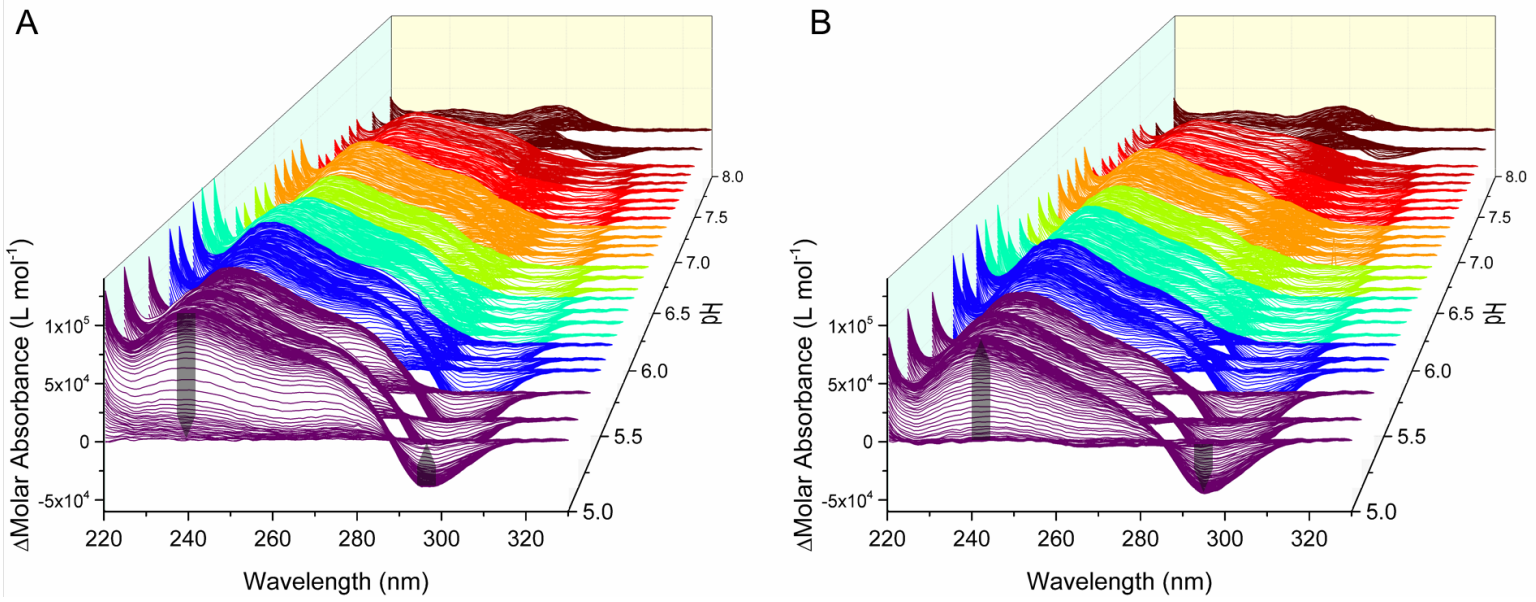

Figure S15.3 The $(\mathbf{A})$ heating and $(\mathbf{B})$ cooling 4DUVMA processes of KRAS: molar absorbance difference, wavelength $(320-220 \mathrm{~nm})$, temperature $\left(5.0-95.0{ }^{\circ} \mathrm{C}\right)$ and $\mathrm{pH}(5.0-8.0)$ The directions of temperature changes (from low to high, and high to low) are denoted with grey arrows in the TDS at pH 5.0.
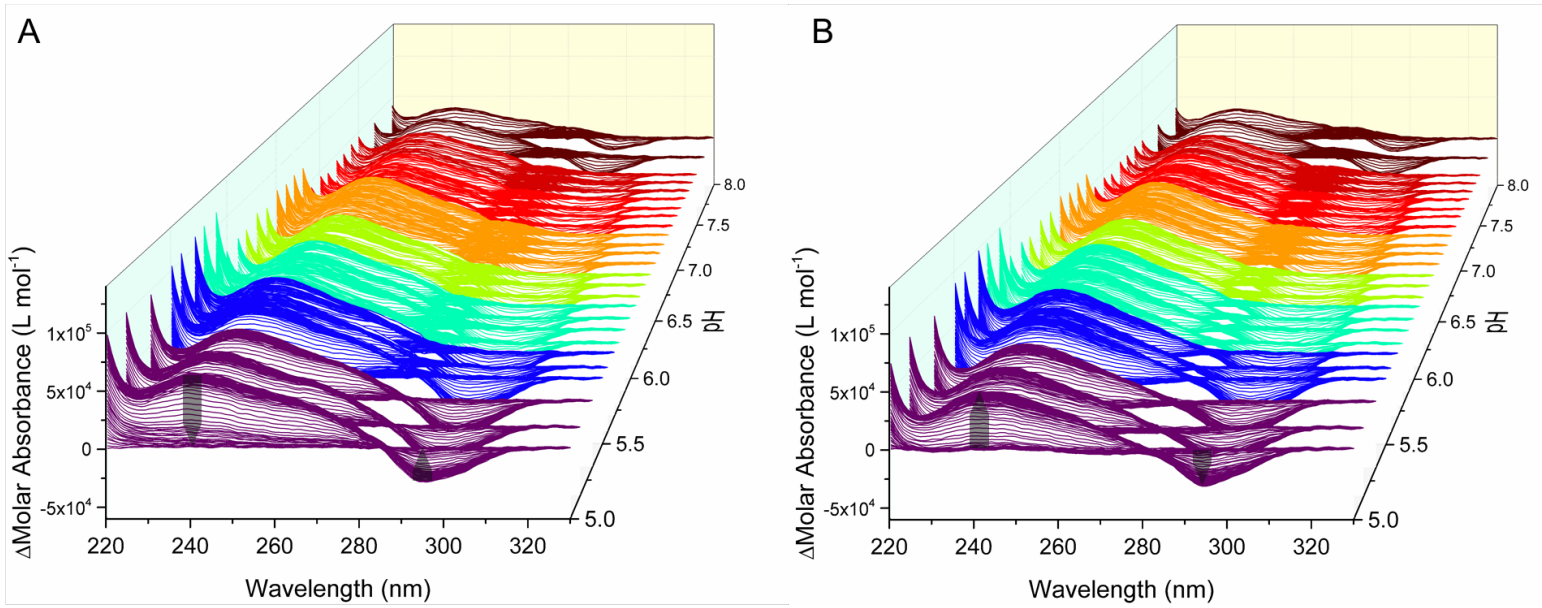

Figure S15.4 The (A) heating and (B) cooling 4DUVMA processes of HIF-1 $\alpha$ : molar absorbance difference, wavelength $(320-220 \mathrm{~nm})$, temperature $\left(5.0-95.0^{\circ} \mathrm{C}\right)$ and $\mathrm{pH}(5.0-8.0)$. The directions of temperature changes (from low to high, and high to low) are denoted with grey arrows in the TDS at pH 5.0.
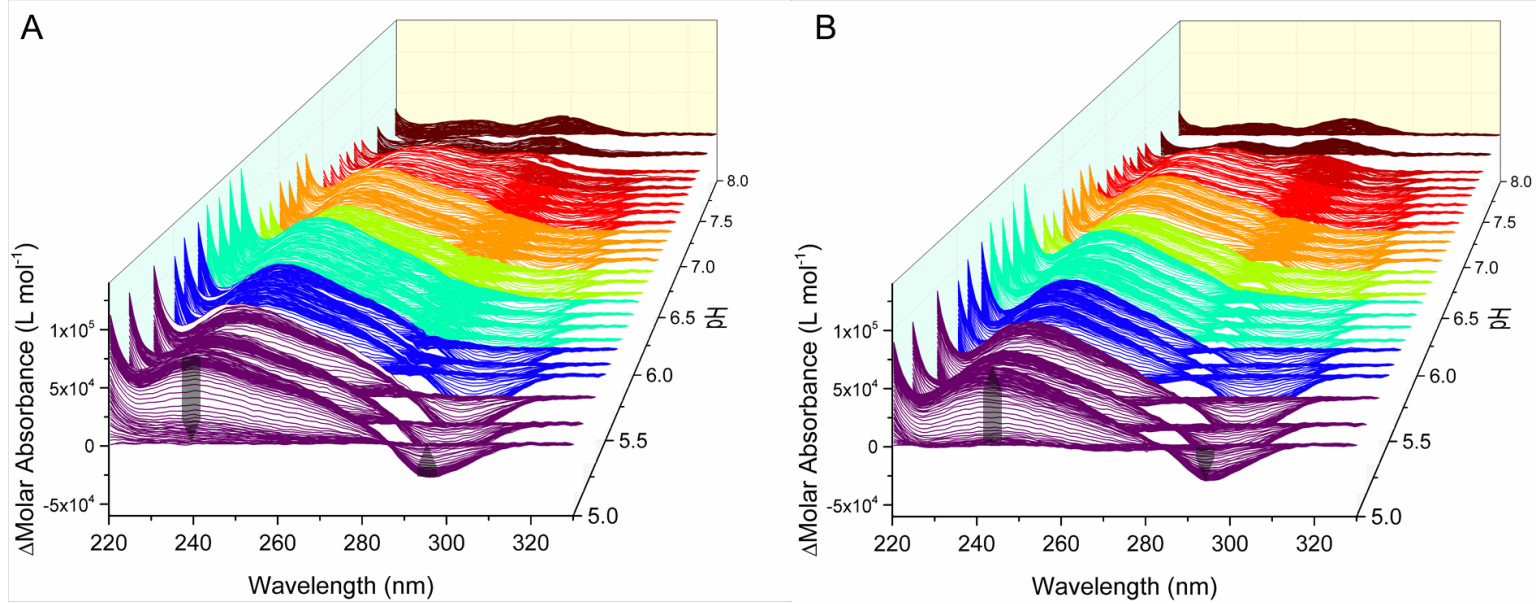

Figure S15.5 The $(\mathbf{A})$ heating and (B) cooling 4DUVMA processes of BCL2: molar absorbance difference, wavelength $(320-220 \mathrm{~nm})$, temperature $\left(5.0-95.0^{\circ} \mathrm{C}\right)$ and $\mathrm{pH}(5.0-8.0)$. The directions of temperature changes (from low to high, and high to low) are denoted with grey arrows in the TDS at pH 5.0. 
Figure S16 Fraction folded of promoter i-DNAs at different pHs and temperatures.
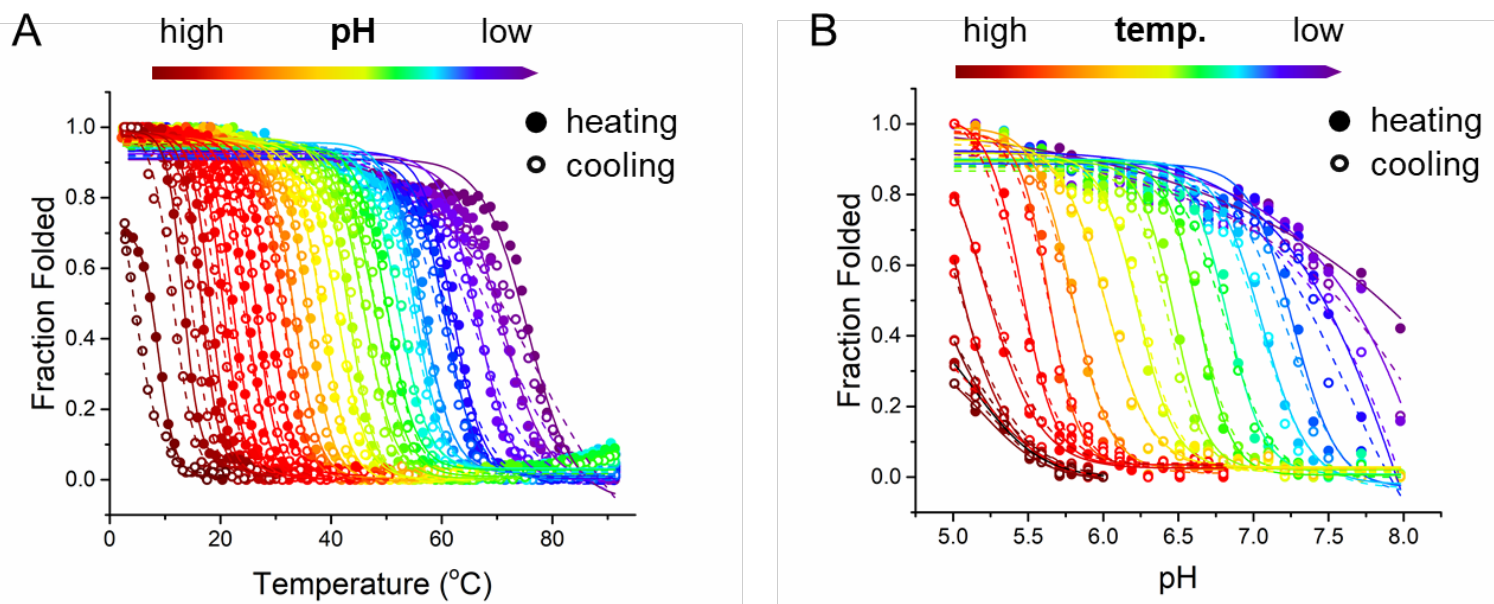

Figure S16.1 (A) i-DNA of DAP fraction folded as a function of temperature deduced from both UV melting and annealing profiles at different pHs. (B) i-DNA faction folded as a function of $\mathrm{pH}$ during both heating and cooling processes.
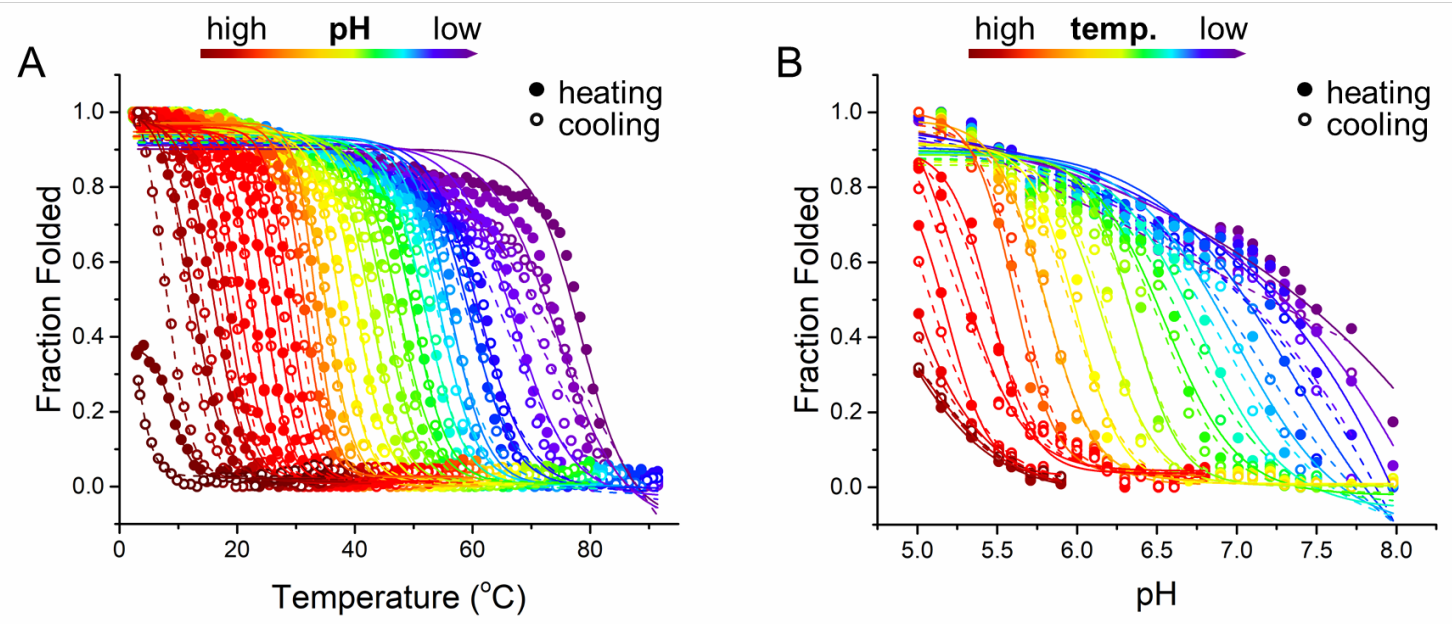

Figure $\mathbf{S 1 6 . 2 ~ ( A ) ~ i - D N A ~ o f ~ P D G F - A ~ f r a c t i o n ~ f o l d e d ~ a s ~ a ~ f u n c t i o n ~ o f ~ t e m p e r a t u r e ~ d e d u c e d ~ f r o m ~ b o t h ~ U V ~}$ melting and annealing profiles at different $\mathrm{pHs}$. (B) i-DNA faction folded as a function of $\mathrm{pH}$ during both heating and cooling processes. 

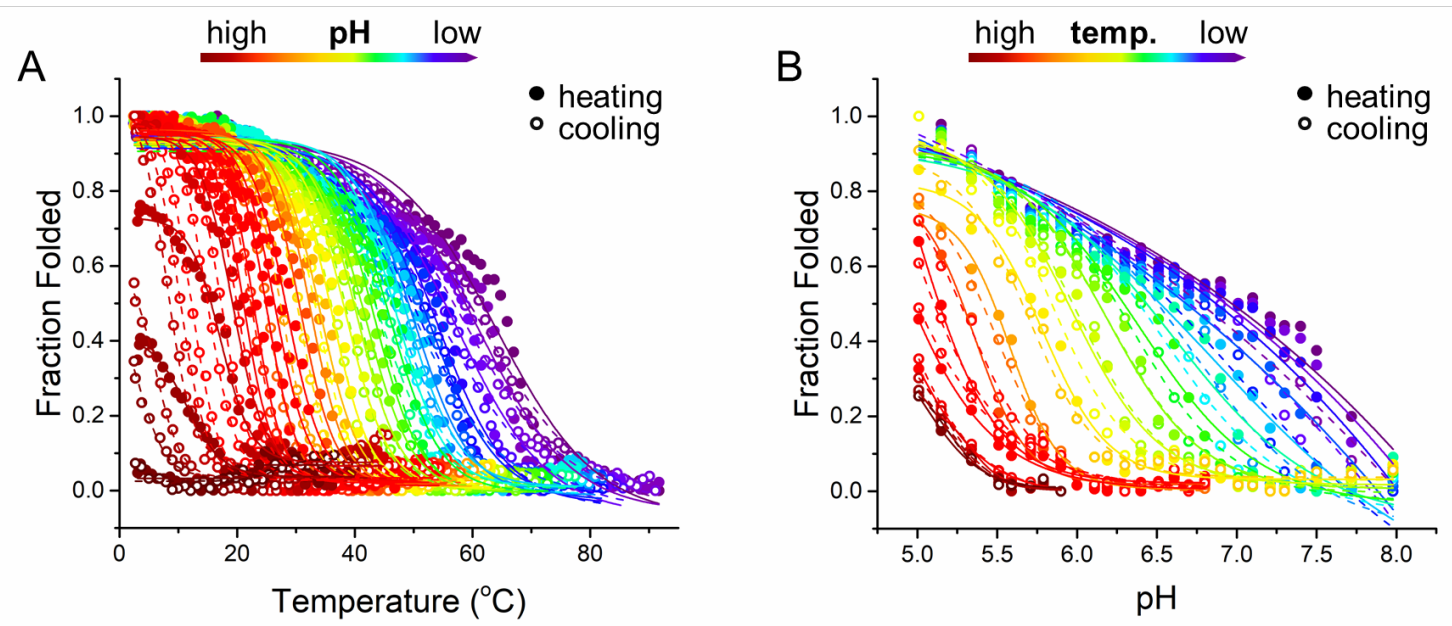

Figure S16.3 (A) i-DNA of KRAS fraction folded as a function of temperature deduced from both UV melting and annealing profiles at different $\mathrm{pHs}$. (B) i-DNA faction folded as a function of $\mathrm{pH}$ during both heating and cooling processes.
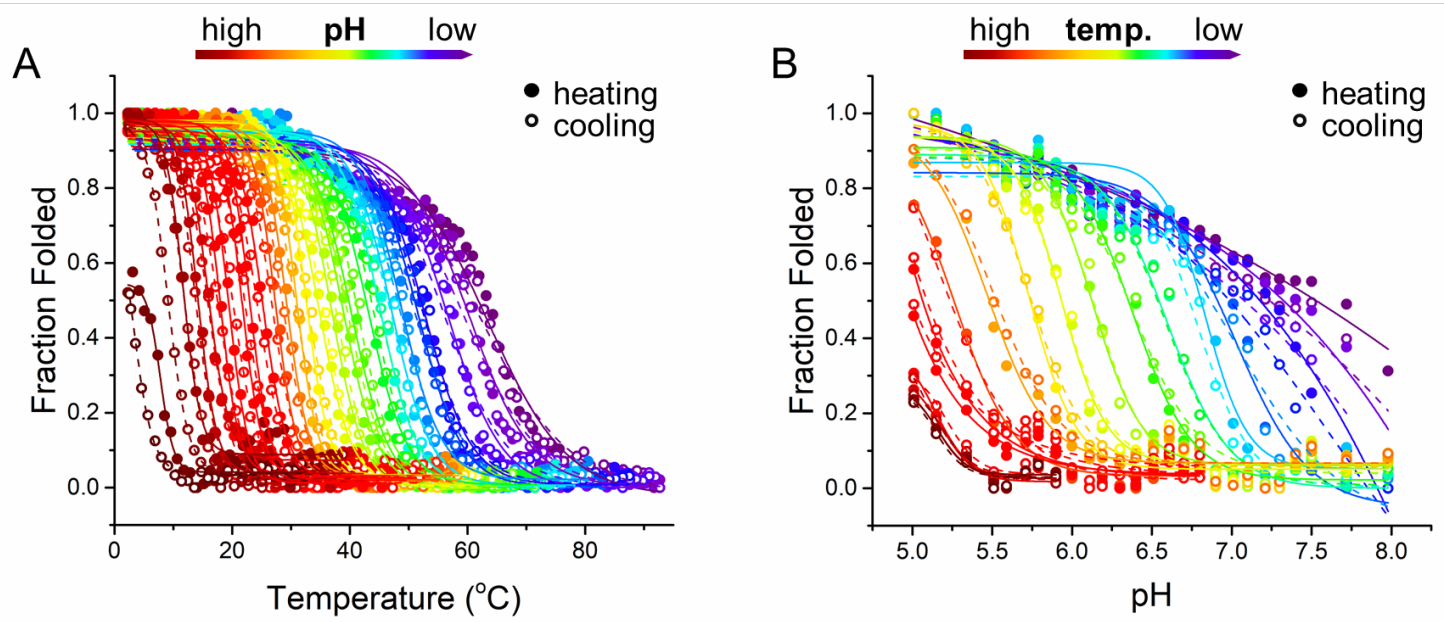

Figure S16.4 (A) i-DNA of HIF-1a fraction folded as a function of temperature deduced from both UV melting and annealing profiles at different $\mathrm{pHs}$. (B) i-DNA faction folded as a function of $\mathrm{pH}$ during both heating and cooling processes.
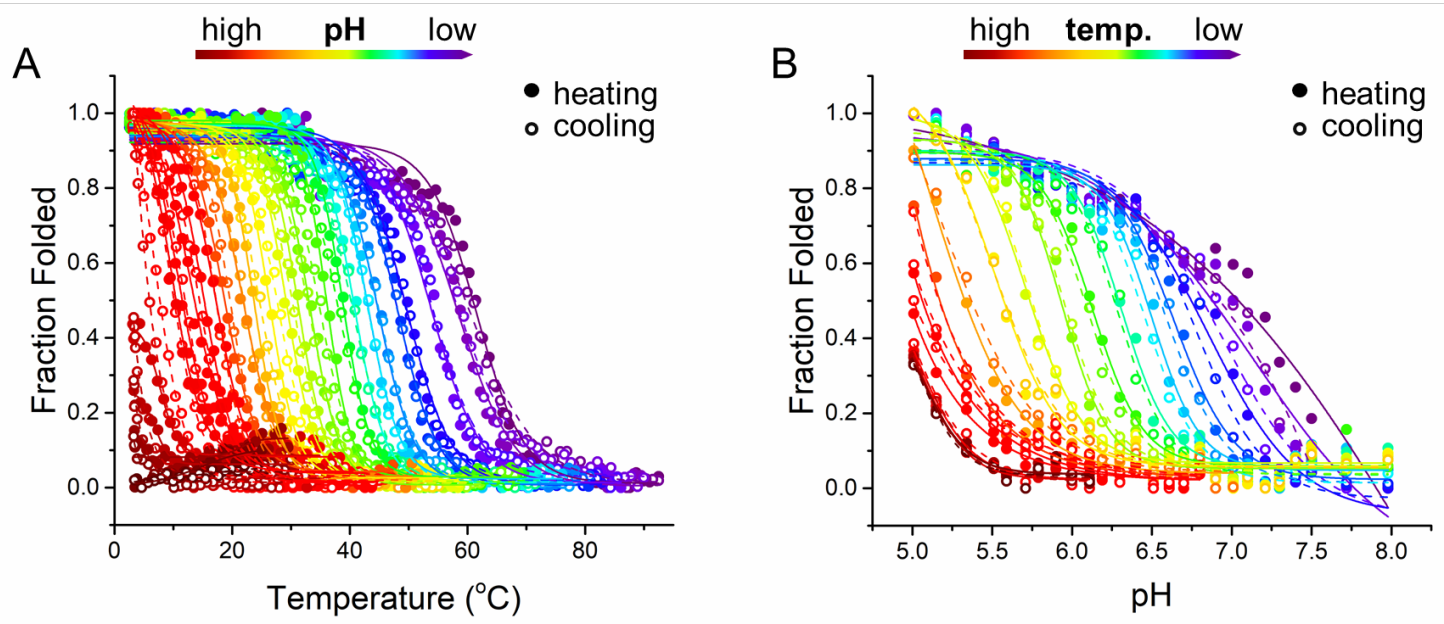

Figure S16.5 (A) i-DNA of BCL2 fraction folded as a function of temperature deduced from both UV melting and annealing profiles at different $\mathrm{pHs}$. (B) i-DNA faction folded as a function of $\mathrm{pH}$ during both heating and cooling processes. 
Figure S17 Formation diagram of promoter i-DNAs at different $\mathrm{pHs}$ and temperatures.

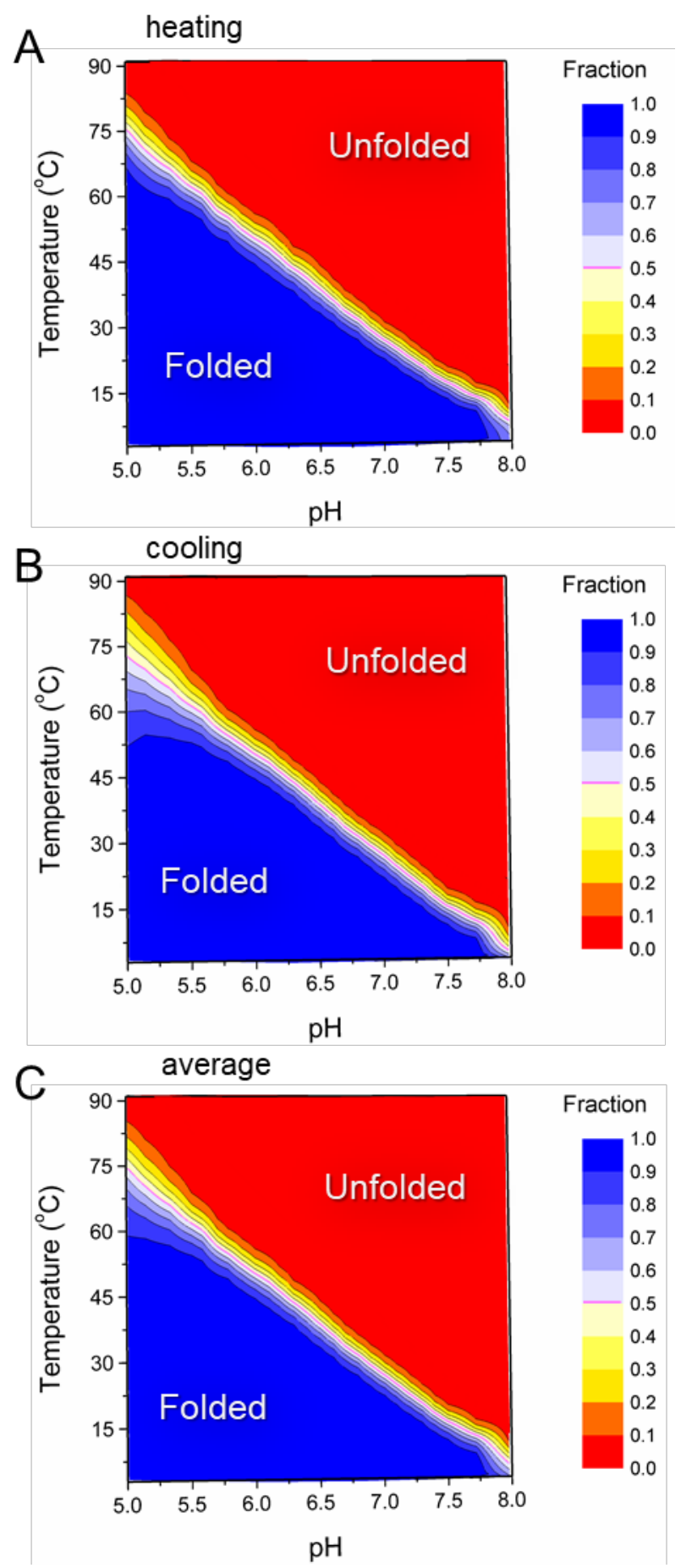

Figure S17.1 i-DNA formation diagram of DAP under varied temperature and $\mathrm{pH}$ combinations during the (A) melting, (B) annealing processes and (C) averaged formation diagram, respectively. 

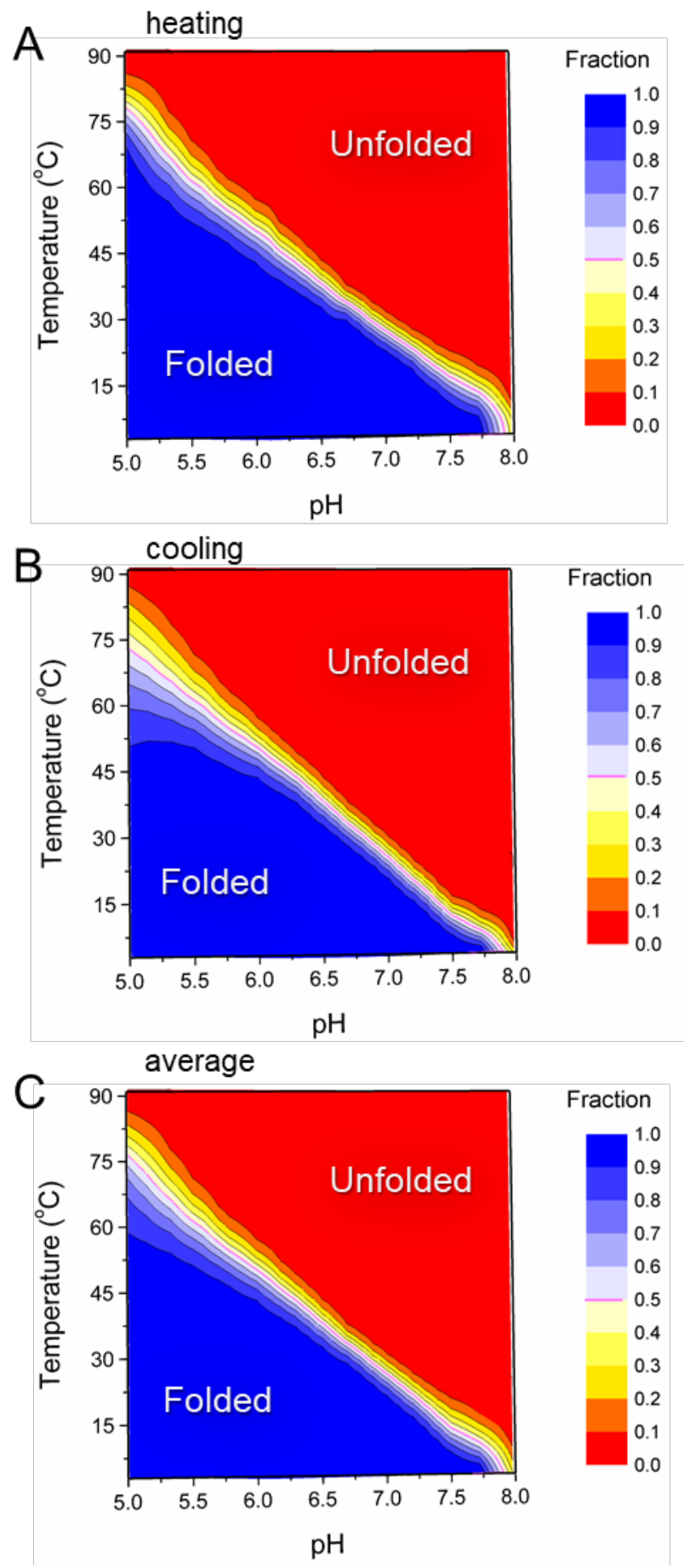

Figure S17.2 i-DNA formation diagram of PDGF-A under varied temperature and $\mathrm{pH}$ combinations during the (A) melting, (B) annealing processes and (C) averaged formation diagram, respectively. 


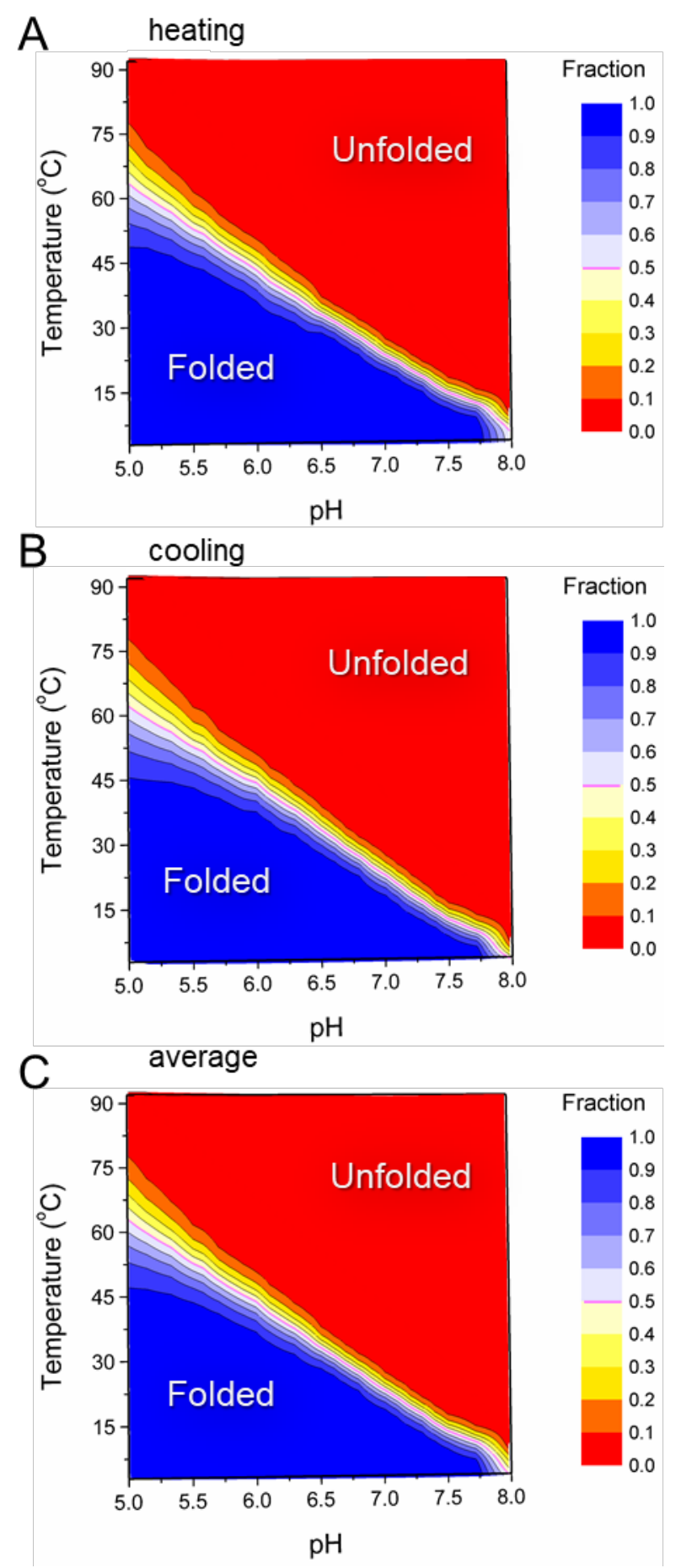

Figure S17.3 i-DNA formation diagram of HIF-1 $\alpha$ under varied temperature and $\mathrm{pH}$ combinations during the (A) melting, (B) annealing processes and (C) averaged formation diagram, respectively. 


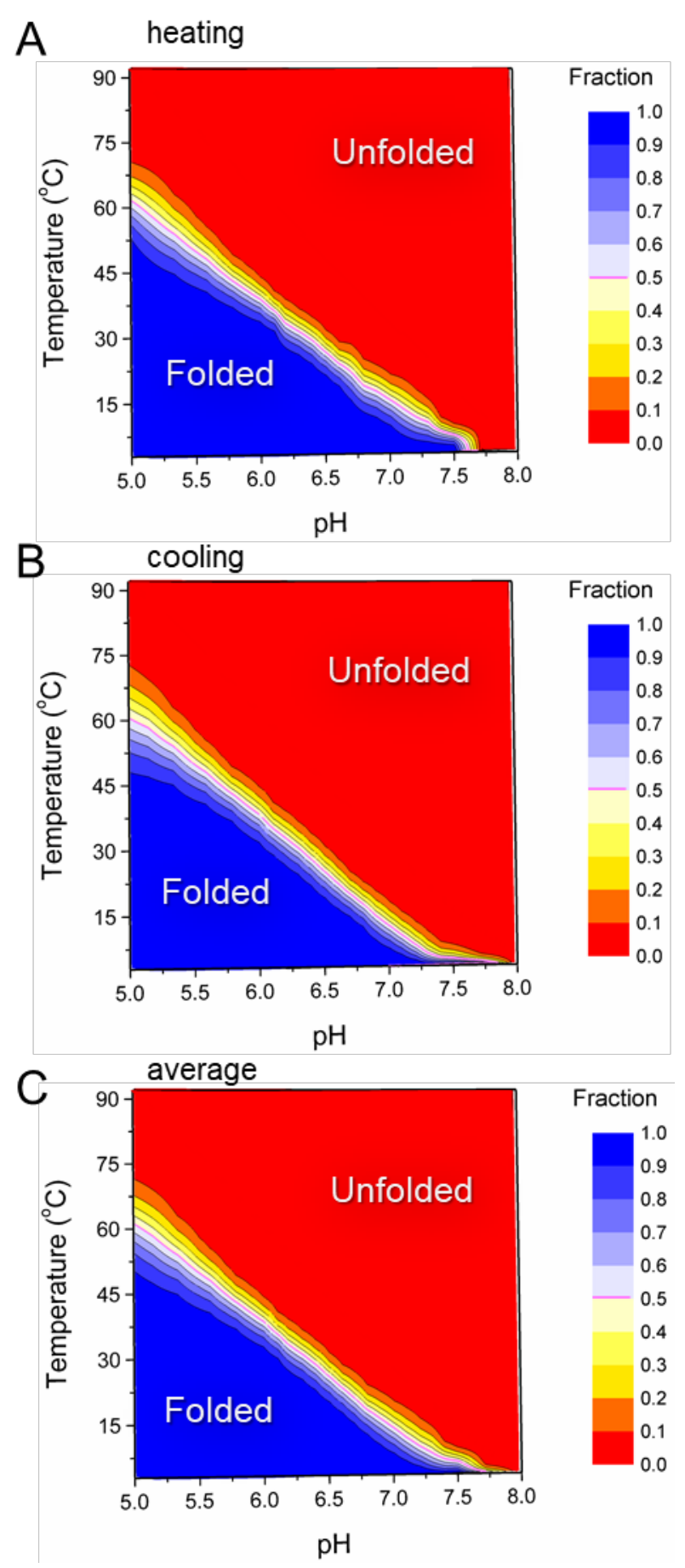

Figure S17.4 i-DNA formation diagram of BCL2 under varied temperature and $\mathrm{pH}$ combinations during the (A) melting, (B) annealing processes and (C) averaged formation diagram, respectively. 
Figure S18 UV-melting and annealing processes of promoter i-DNAs under $\mathrm{pH} 7.0$ without or with crowding agents.
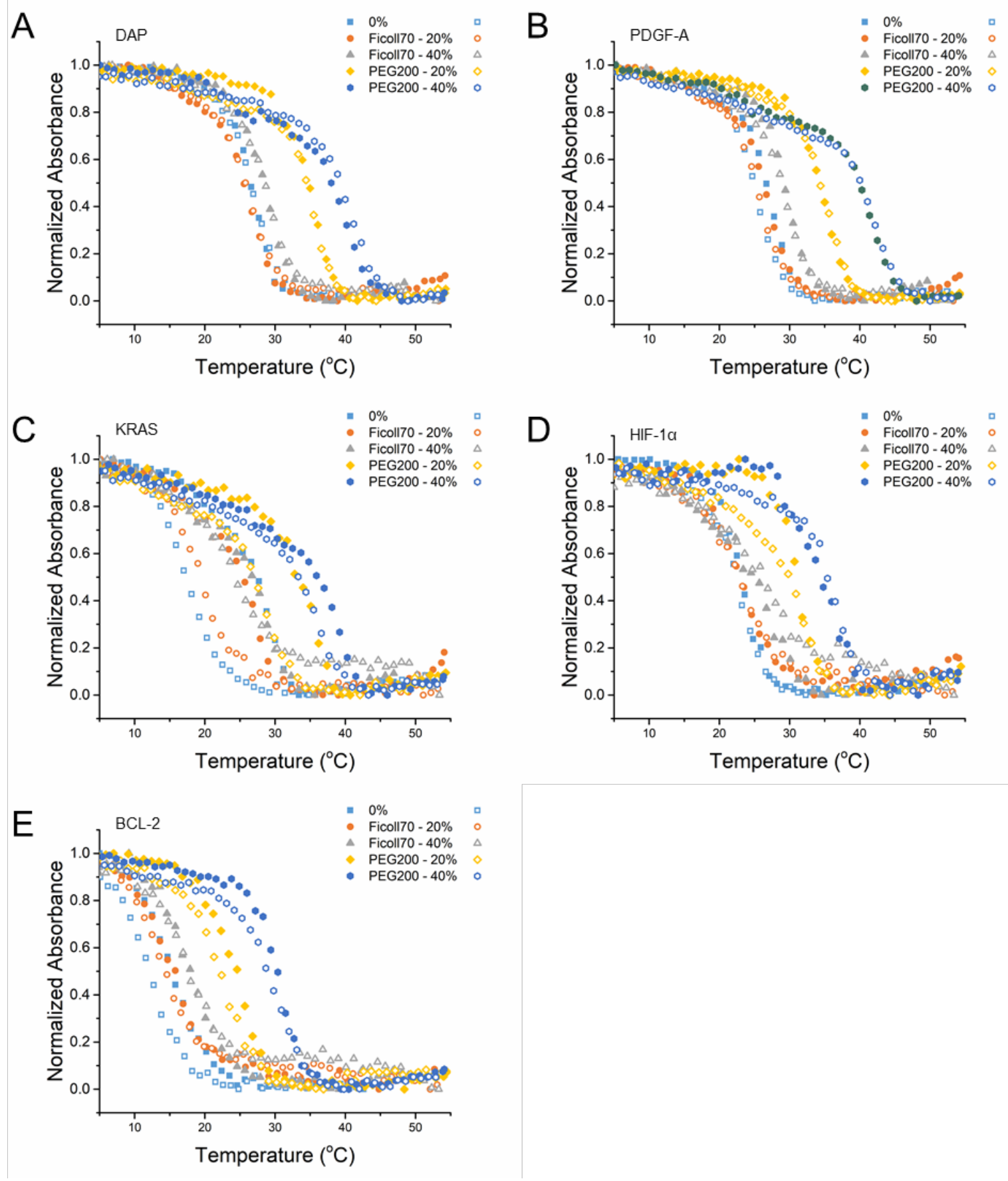

Figure S18 UV-melting (filled circles) and annealing (Hollow circles) under $\mathrm{pH} 7.0$ without or with various crowding agents: (A) DAP, (B) PDGF-A, (C) KRAS, (D) HIF-1a, and (E) BCL2. 
Figure $\mathbf{S} 19$ Standard Gibbs free energy $\left(\Delta G^{\circ}\right)$ at $20^{\circ} \mathrm{C}$ of promoter i-DNAs.

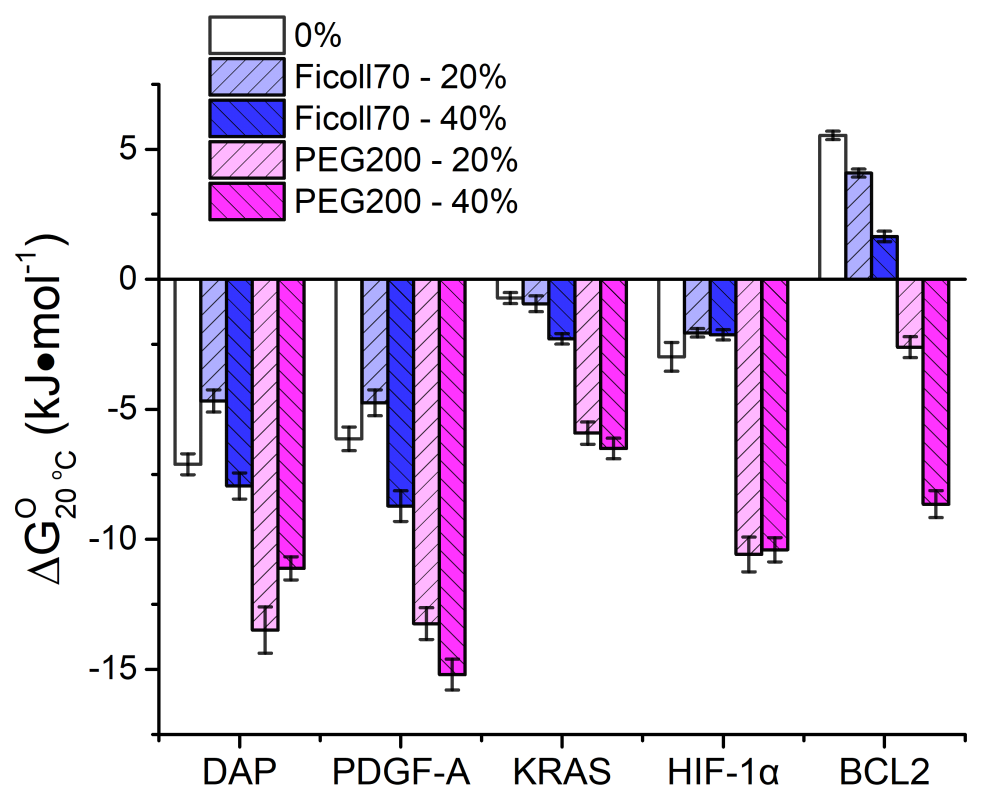

Figure S19 Standard Gibbs free energy $\left(\Delta G^{\circ}\right)$ at $20^{\circ} \mathrm{C}$ under neutral pH (7.0) and crowding environments. 
Table S1 $T_{1 / 2}$ obtained from UV melting and annealing processes at different $\mathrm{pHs}$.

\begin{tabular}{|c|c|c|c|c|c|c|c|c|c|c|c|c|c|c|c|c|}
\hline \multirow{3}{*}{$\begin{array}{c}\mathrm{pH} \\
\text { Oligo }\end{array}$} & \multicolumn{4}{|c|}{ pH 5.01} & \multicolumn{4}{|c|}{ pH 5.15} & \multicolumn{4}{|c|}{ pH 5.34} & \multicolumn{4}{|c|}{ pH 5.51} \\
\hline & \multicolumn{2}{|c|}{ heating } & \multicolumn{2}{|c|}{ cooling } & \multicolumn{2}{|c|}{ heating } & \multicolumn{2}{|c|}{ cooling } & \multicolumn{2}{|c|}{ heating } & \multicolumn{2}{|c|}{ cooling } & \multicolumn{2}{|c|}{ heating } & \multicolumn{2}{|c|}{ cooling } \\
\hline & $\begin{array}{c}\mathrm{T}_{1 / 2}, \\
{ }^{\circ} \mathrm{C}\end{array}$ & $\begin{array}{l}\text { SE, } \\
{ }^{\circ} \mathrm{C}\end{array}$ & $\begin{array}{l}\mathrm{T}_{1 / 2} \\
{ }^{\circ} \mathrm{C}\end{array}$ & $\begin{array}{l}\text { SE, } \\
{ }^{\circ} \mathrm{C}\end{array}$ & $\begin{array}{l}\mathrm{T}_{1 / 2} \\
{ }^{\circ} \mathrm{C}\end{array}$ & $\begin{array}{l}\text { SE, } \\
{ }^{\circ} \mathrm{C}\end{array}$ & $\begin{array}{c}\mathrm{T}_{1 / 2}, \\
{ }^{\circ} \mathrm{C}\end{array}$ & $\begin{array}{l}\text { SE, } \\
{ }^{\circ} \mathrm{C}\end{array}$ & $\begin{array}{c}\mathrm{T}_{1 / 2}, \\
{ }^{\circ} \mathrm{C}\end{array}$ & $\begin{array}{l}\text { SE, } \\
{ }^{\circ} \mathrm{C}\end{array}$ & $\begin{array}{c}\mathrm{T}_{1 / 2} \\
{ }^{\circ} \mathrm{C}\end{array}$ & $\begin{array}{l}\text { SE, } \\
{ }^{\circ} \mathrm{C}\end{array}$ & $\begin{array}{l}\mathrm{T}_{1 / 2} \\
{ }^{\circ} \mathrm{C}\end{array}$ & $\begin{array}{l}\text { SE, } \\
{ }^{\circ} \mathrm{C}\end{array}$ & $\begin{array}{c}\mathrm{T}_{1 / 2} \\
{ }^{\circ} \mathrm{C}\end{array}$ & $\begin{array}{l}\text { SE, } \\
{ }^{\circ} \mathrm{C}\end{array}$ \\
\hline 33L1 & 54.0 & 0.2 & 51.4 & 0.4 & 51.7 & 0.1 & 47.8 & 0.1 & 47.2 & 0.1 & 44.0 & 0.1 & 43.3 & 0.2 & 38.1 & 0.1 \\
\hline 33L3 & 54.4 & 0.4 & 53.1 & 0.5 & 52.9 & 0.3 & 52.7 & 0.2 & 48.8 & 0.1 & 49.3 & 0.1 & 45.8 & 0.1 & 45.5 & 0.1 \\
\hline 34L1 & 54.3 & 0.4 & 53.1 & 0.5 & 52.8 & 0.2 & 50.2 & 0.3 & 47.0 & 0.2 & 46.3 & 0.1 & 43.1 & 0.2 & 41.5 & 0.1 \\
\hline $34 \mathrm{~L} 3$ & 61.9 & 0.3 & 59.7 & 0.2 & 56.4 & 0.1 & 55.9 & 0.2 & 53.3 & 0.1 & 52.9 & 0.1 & 48.4 & 0.1 & 48.9 & 0.1 \\
\hline 43L1 & 59.5 & 0.2 & 58.5 & 0.2 & 55.6 & 0.1 & 55.1 & 0.1 & 51.3 & 0.1 & 51.9 & 0.1 & 47.3 & 0.1 & 47.4 & 0.1 \\
\hline $43 L 3$ & 62.6 & 0.2 & 61.0 & 0.3 & 59.6 & 0.1 & 58.9 & 0.1 & 71.5 & 0.3 & 72.2 & 0.3 & 51.5 & 0.1 & 52.0 & 0.1 \\
\hline 44L1 & 62.7 & 0.3 & 60.9 & 0.3 & 59.5 & 0.2 & 58.2 & 0.2 & 71.1 & 0.1 & 71.5 & 0.2 & 50.0 & 0.1 & 50.1 & 0.1 \\
\hline $44 \mathrm{~L} 3$ & 66.4 & 0.2 & 64.7 & 0.3 & 62.8 & 0.1 & 62.6 & 0.2 & 72.5 & 0.4 & 73.2 & 0.4 & 54.6 & 0.1 & 54.9 & 0.1 \\
\hline 45L1 & 66.1 & 0.3 & 64.2 & 0.4 & 62.3 & 0.2 & 61.9 & 0.3 & 72.4 & 0.1 & 72.8 & 0.2 & 53.3 & 0.1 & 53.3 & 0.1 \\
\hline $45 \mathrm{~L} 3$ & 69.2 & 0.2 & 68.9 & 0.3 & 64.9 & 0.1 & 64.7 & 0.2 & 47.2 & 0.1 & 44.0 & 0.1 & 57.6 & 0.1 & 56.4 & 0.1 \\
\hline 54L1 & 70.6 & 0.1 & 69.5 & 0.3 & 66.9 & 0.1 & 65.8 & 0.1 & 48.7 & 0.1 & 49.3 & 0.1 & 55.9 & 0.1 & 56.1 & 0.1 \\
\hline $54 \mathrm{~L} 3$ & 71.0 & 0.1 & 70.3 & 0.4 & 66.4 & 0.2 & 66.2 & 0.3 & 47.0 & 0.2 & 46.3 & 0.1 & 58.0 & 0.1 & 57.8 & 0.1 \\
\hline $55 \mathrm{~L} 1$ & 70.3 & 0.3 & 69.7 & 0.4 & 65.8 & 0.2 & 66.1 & 0.2 & 52.1 & 0.1 & 52.5 & 0.1 & 57.7 & 0.1 & 57.8 & 0.2 \\
\hline $55 \mathrm{~L} 3$ & 71.8 & 0.1 & 71.1 & 0.2 & 67.6 & 0.1 & 67.4 & 0.1 & 51.3 & 0.1 & 51.9 & 0.1 & 59.8 & 0.2 & 60.0 & 0.2 \\
\hline 56L1 & 73.0 & 0.3 & 71.4 & 0.4 & 67.7 & 0.2 & 67.4 & 0.3 & 64.0 & 0.2 & 63.9 & 0.3 & 59.7 & 0.2 & 60.0 & 0.3 \\
\hline $56 \mathrm{~L} 3$ & 72.6 & 0.1 & 71.5 & 0.2 & 68.7 & 0.1 & 68.3 & 0.1 & 65.2 & 0.1 & 64.8 & 0.2 & 61.0 & 0.1 & 60.5 & 0.1 \\
\hline 65L1 & 75.5 & 0.2 & 74.1 & 0.3 & 71.4 & 0.4 & 72.2 & 0.4 & 68.4 & 0.2 & 66.6 & 0.2 & 62.1 & 0.1 & 62.0 & 0.2 \\
\hline $65 \mathrm{~L} 3$ & 74.2 & 0.1 & 73.1 & 0.2 & 71.1 & 0.1 & 71.5 & 0.2 & 68.4 & 0.1 & 66.5 & 0.2 & 62.4 & 0.1 & 62.1 & 0.1 \\
\hline 66L1 & 76.4 & 0.7 & 77.2 & 1.2 & 72.5 & 0.4 & 73.2 & 0.4 & 69.2 & 0.3 & 67.3 & 0.3 & 62.6 & 0.3 & 62.6 & 0.2 \\
\hline $66 \mathrm{~L} 3$ & 75.7 & 0.2 & 74.8 & 0.5 & 72.4 & 0.1 & 72.8 & 0.2 & 67.4 & 0.1 & 66.9 & 0.2 & 63.4 & 0.1 & 62.7 & 0.2 \\
\hline
\end{tabular}

"SE" is standard error. Thermal and $\mathrm{pH}$ transitions were both analyzed with nonlinear regression using Boltzmann sigmoidal equation and assuming a two-state transition model. An accurate determination of the pre- and post-transition baselines is critical. Whenever the $T_{m}$ is too low, or $p H_{T}$ is too low or too high, there is no clear pre-baseline or post-baseline, respectively. For this reason, we did not consider these fits as reliable. This is illustrated in Figures 5B, 6B and Figures 7A-D, A few $T_{1 / 2}$ and $p H_{T}$ values are missing due to the lack of obvious pre- or post-transition baselines: ${ }^{a}$ indicates that the quality of the data is not sufficient to allow an accurate determination, or that the stability is too low to determine $T_{1 / 2}$. 
Table S1 $T_{1 / 2}$ obtained from UV melting and annealing processes at different pHs. (Continued_01)

\begin{tabular}{|c|c|c|c|c|c|c|c|c|c|c|c|c|c|c|c|c|}
\hline \multirow{3}{*}{$\begin{array}{c}\mathrm{pH} \\
\text { Oligo }\end{array}$} & \multicolumn{4}{|c|}{ pH 5.59} & \multicolumn{4}{|c|}{ pH 5.71} & \multicolumn{4}{|c|}{ pH 5.79} & \multicolumn{4}{|c|}{ pH 5.90} \\
\hline & \multicolumn{2}{|c|}{ heating } & \multicolumn{2}{|c|}{ cooling } & \multicolumn{2}{|c|}{ heating } & \multicolumn{2}{|c|}{ cooling } & \multicolumn{2}{|c|}{ heating } & \multicolumn{2}{|c|}{ cooling } & \multicolumn{2}{|c|}{ heating } & \multicolumn{2}{|c|}{ cooling } \\
\hline & $\begin{array}{c}\mathrm{T}_{1 / 2} \\
{ }^{\circ} \mathrm{C}\end{array}$ & $\begin{array}{l}\mathrm{SE}, \\
{ }^{\circ} \mathrm{C}\end{array}$ & $\begin{array}{c}\mathrm{T}_{1 / 2} \\
{ }^{\circ} \mathrm{C} \\
\end{array}$ & $\begin{array}{c}\text { SE, } \\
{ }^{\circ} \mathrm{C}\end{array}$ & $\begin{array}{c}\mathrm{T}_{1 / 2} \\
{ }^{\circ} \mathrm{C} \\
\end{array}$ & $\begin{array}{c}\text { SE, } \\
{ }^{\circ} \mathrm{C}\end{array}$ & $\begin{array}{c}\mathrm{T}_{1 / 2} \\
{ }^{\circ} \mathrm{C} \\
\end{array}$ & $\begin{array}{l}\text { SE, } \\
{ }^{\circ} \mathrm{C}\end{array}$ & $\begin{array}{c}\mathrm{T}_{1 / 2}, \\
{ }^{\circ} \mathrm{C}\end{array}$ & $\begin{array}{l}\text { SE, } \\
{ }^{\circ} \mathrm{C}\end{array}$ & $\begin{array}{c}\mathrm{T}_{1 / 2} \\
{ }^{\circ} \mathrm{C}\end{array}$ & $\begin{array}{l}\mathrm{SE}, \\
{ }^{\circ} \mathrm{C}\end{array}$ & $\begin{array}{c}\mathrm{T}_{1 / 2} \\
{ }^{\circ} \mathrm{C}\end{array}$ & $\begin{array}{c}\text { SE, } \\
{ }^{\circ} \mathrm{C}\end{array}$ & $\begin{array}{c}\mathrm{T}_{1 / 2} \\
{ }^{\circ} \mathrm{C} \\
\end{array}$ & $\begin{array}{l}\text { SE, } \\
{ }^{\circ} \mathrm{C}\end{array}$ \\
\hline 33L1 & 41.8 & 0.2 & 36.6 & 0.1 & 40.6 & 0.1 & 33.0 & 0.1 & 39.2 & 0.1 & 31.1 & 0.1 & 36.9 & 0.2 & 27.8 & 0.2 \\
\hline 33L3 & 43.5 & 0.1 & 43.8 & 0.1 & 41.2 & 0.1 & 40.6 & 0.1 & 39.1 & 0.1 & 39.5 & 0.1 & 7.5 & 0.1 & 37.3 & 0.1 \\
\hline 34L1 & 41.5 & 0.2 & 39.0 & 0.1 & 38.4 & 0.2 & 36.0 & 0.2 & 35.8 & 0.3 & 34.2 & 0.2 & 34.1 & 0.2 & 32.6 & 0.2 \\
\hline $34 \mathrm{~L} 3$ & 46.4 & 0.1 & 46.8 & 0.1 & 43.8 & 0.1 & 44.0 & 0.1 & 41.8 & 0.1 & 42.0 & 0.1 & 40.5 & 0.1 & 40.2 & 0.1 \\
\hline 43L1 & 45.3 & 0.1 & 45.3 & 0.1 & 41.8 & 0.1 & 41.4 & 0.2 & 40.0 & 0.1 & 40.3 & 0.1 & 7.9 & 0.1 & 38.0 & 1 \\
\hline 43L3 & 50.0 & 0.1 & 50.4 & 0.1 & 46.9 & 0.1 & 47.3 & 0.1 & 45.3 & 0.1 & 45.5 & 0.1 & 44.4 & 0.1 & 43.8 & 0.1 \\
\hline 44L1 & 48.0 & 0.1 & 47.6 & 0.1 & 44.5 & 0.1 & 44.8 & 0.1 & 44.1 & 0.2 & 42.7 & 0.2 & 40.0 & 0.2 & 40.4 & 0.1 \\
\hline 44L3 & 52.5 & 0.1 & 52.8 & 0.1 & 49.6 & 0.1 & 48.9 & 0.1 & 48.2 & 0.1 & 48.4 & 0.1 & 45.9 & 0.1 & 46.0 & 0.1 \\
\hline 45L1 & 51.5 & 0.1 & 51.8 & 0.1 & 47.9 & 0. & 48.1 & 0. & 45.6 & 0. & 46.6 & 0.2 & 44.0 & 0.1 & 44.4 & 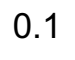 \\
\hline $45 \mathrm{~L} 3$ & 54.4 & 0.1 & 54.6 & 0.1 & 52.9 & 0.1 & 71.4 & 0.1 & 49.6 & 0.3 & 50.2 & 0.3 & 47.5 & 0.1 & 47.5 & 0.1 \\
\hline 54L1 & 54.2 & 0.1 & 54.5 & 0.1 & 52.8 & 0.1 & 71.4 & 0.1 & 49.4 & 0.1 & 49.5 & 0.1 & 46.8 & 0.1 & 47.2 & 0.1 \\
\hline $54 \mathrm{~L} 3$ & 56.0 & 0.1 & 56.1 & 0.1 & 55.2 & 0.1 & 73.7 & 0.1 & 51.5 & 0.1 & 51.6 & 0.1 & 49.8 & 0.1 & 49.6 & 0.1 \\
\hline $55 \mathrm{~L} 1$ & 55.8 & 0.1 & 56.1 & 0.2 & 54.6 & 0.1 & 73.2 & 0.1 & 50.7 & 0.2 & 50.8 & 0.1 & 47.9 & 0.1 & 48.7 & 0.1 \\
\hline $55 \mathrm{~L} 3$ & 58.2 & 0.1 & 58.1 & 0.1 & 54.9 & 0.1 & 54.8 & 0.1 & 53.3 & 0.1 & 52.8 & 0.1 & 50.7 & 0.1 & 50.7 & 0.1 \\
\hline $56 \mathrm{~L} 1$ & 58.1 & 0.2 & 58.2 & 0.2 & 54.3 & 0.1 & 54.6 & 0.2 & 53.1 & 0.1 & 53.0 & 0.2 & 50.6 & 0.1 & 50.6 & 0.2 \\
\hline 56 L3 & 59.0 & 0.1 & 58.7 & 0.1 & 55.6 & 0.1 & 55.2 & 0.1 & 54.0 & 0.1 & 53.8 & 0.1 & 51.9 & 0.1 & 51.4 & 0.1 \\
\hline 65L1 & 59.8 & 0.1 & 59.9 & 0.1 & 56.4 & 0.1 & 56.6 & 0.1 & 54.6 & 0.1 & 54.4 & 0.1 & 52.9 & 0.2 & 52.9 & 0.2 \\
\hline $65 \mathrm{~L} 3$ & 60.8 & 0.1 & 60.1 & 0.1 & 57.6 & 0.1 & 57.1 & 0.1 & 55.4 & 0.1 & 55.2 & 0.1 & 53.7 & 0.1 & 52.5 & 0.1 \\
\hline 66L1 & 60.3 & 0.2 & 60.8 & 0.2 & 57.3 & 0.3 & 57.1 & 0.4 & 55.5 & 0.2 & 55.9 & 0.2 & 53.2 & 0.2 & 53.3 & 0.2 \\
\hline $66 \mathrm{~L} 3$ & 61.7 & 0.1 & 60.7 & 0.1 & 59.0 & 0.1 & 57.5 & 0.2 & 57.0 & 0.1 & 55.2 & 0.1 & 54.8 & 0.1 & 52.6 & 0.1 \\
\hline
\end{tabular}

SE, standard error;

${ }^{a}$ indicates that the quality of the data is not sufficient to allow an accurate determination, or that the stability is too low to determine $T_{1 / 2}$. 
Table S1 $T_{1 / 2}$ obtained from UV melting and annealing processes at different pHs. (Continued_02)

\begin{tabular}{|c|c|c|c|c|c|c|c|c|c|c|c|c|c|c|c|c|}
\hline \multirow{3}{*}{$\begin{array}{c}\mathrm{pH} \\
\text { Oligo }\end{array}$} & \multicolumn{4}{|c|}{ pH 6.00} & \multicolumn{4}{|c|}{ pH 6.11} & \multicolumn{4}{|c|}{ pH 6.19} & \multicolumn{4}{|c|}{ pH 6.30} \\
\hline & \multicolumn{2}{|c|}{ heating } & \multicolumn{2}{|c|}{ cooling } & \multicolumn{2}{|c|}{ heating } & \multicolumn{2}{|c|}{ cooling } & \multicolumn{2}{|c|}{ heating } & \multicolumn{2}{|c|}{ cooling } & \multicolumn{2}{|c|}{ heating } & \multicolumn{2}{|c|}{ cooling } \\
\hline & $\begin{array}{l}\mathrm{T}_{1 / 2} \\
{ }^{\circ} \mathrm{C}\end{array}$ & $\begin{array}{l}\text { SE, } \\
{ }^{\circ} \mathrm{C}\end{array}$ & $\begin{array}{c}\mathrm{T}_{1 / 2} \\
{ }^{\circ} \mathrm{C}\end{array}$ & $\begin{array}{l}\text { SE, } \\
{ }^{\circ} \mathrm{C}\end{array}$ & $\begin{array}{c}\mathrm{T}_{1 / 2} \\
{ }^{\circ} \mathrm{C}\end{array}$ & $\begin{array}{l}\text { SE, } \\
{ }^{\circ} \mathrm{C}\end{array}$ & $\begin{array}{c}\mathrm{T}_{1 / 2}, \\
{ }^{\circ} \mathrm{C}\end{array}$ & $\begin{array}{l}\text { SE, } \\
{ }^{\circ} \mathrm{C}\end{array}$ & $\begin{array}{c}\mathrm{T}_{1 / 2} \\
{ }^{\circ} \mathrm{C}\end{array}$ & $\begin{array}{l}\text { SE, } \\
{ }^{\circ} \mathrm{C}\end{array}$ & $\begin{array}{c}\mathrm{T}_{1 / 2} \\
{ }^{\circ} \mathrm{C}\end{array}$ & $\begin{array}{l}\mathrm{SE}, \\
{ }^{\circ} \mathrm{C}\end{array}$ & $\begin{array}{c}\mathrm{T}_{1 / 2} \\
{ }^{\circ} \mathrm{C}\end{array}$ & $\begin{array}{l}\text { SE, } \\
{ }^{\circ} \mathrm{C}\end{array}$ & $\begin{array}{c}\mathrm{T}_{1 / 2} \\
{ }^{\circ} \mathrm{C}\end{array}$ & $\begin{array}{l}\text { SE, } \\
{ }^{\circ} \mathrm{C}\end{array}$ \\
\hline 33L1 & 33.5 & 0.1 & 25.9 & 0.1 & 32.0 & 0.2 & 22.2 & 0.1 & 29.4 & 0.1 & 20.4 & 0.1 & 27.4 & 0.3 & 17.4 & 0.3 \\
\hline 33L3 & 35.2 & 0.1 & 35.7 & 0.1 & 31.9 & 0.1 & 32.8 & 0.1 & 30.2 & 0.1 & 31.1 & 0.1 & 28.6 & 0.1 & 29.0 & 0.1 \\
\hline 34L1 & 34.4 & 0.1 & 30.6 & 0.1 & 30.3 & 0.2 & 27.6 & 0.1 & 27.0 & 0.3 & 25.6 & 0.1 & 25.8 & 0.3 & 22.9 & 0.2 \\
\hline 34L3 & 37.9 & 0.1 & 38.3 & 0.1 & 35.0 & 0.1 & 35.2 & 0.1 & 33.5 & 0.1 & 33.1 & 0.1 & 30.5 & 0.1 & 31.1 & 0.1 \\
\hline 43L1 & 36.0 & 0.1 & 36.2 & 0.1 & .9 & 0.1 & 3.0 & 0.1 & 30.8 & 0.2 & 31.0 & 1 & .1 & 0.1 & 28.8 & 0.1 \\
\hline $43 L 3$ & 41.5 & 0.1 & 41.9 & 0.1 & 38.2 & 0.1 & 39.0 & 0.1 & 36.3 & 0.1 & 37.3 & 0.1 & 35.0 & 0.1 & 35.3 & 0.1 \\
\hline 44L1 & 38.3 & 0.1 & 38.7 & 0.1 & 35.0 & 0.1 & 35.5 & 0.1 & 33.5 & 0.1 & 33.4 & 0.1 & 31.4 & 0.1 & 31.2 & 0.1 \\
\hline 44L3 & 43.9 & 0.1 & 44.2 & 0.1 & 40.6 & 0.1 & 41.3 & 0.1 & 38.5 & 0.1 & 39.2 & 0.1 & 6.9 & 0.1 & 36.9 & 0.1 \\
\hline 45L1 & 42.0 & 0.1 & 42.5 & 0.1 & 38.4 & 0.1 & 39.4 & 0.1 & 36.4 & 0. & 37.3 & 0.1 & . & 0.1 & 34.8 & 0.1 \\
\hline $45 \mathrm{~L} 3$ & 45.5 & 0.1 & 45.8 & 0.0 & 42.2 & 0.1 & 42.5 & 0.0 & 40.1 & 0.1 & 40.5 & 0.1 & 38.7 & 0.1 & 38.2 & 0.1 \\
\hline 54L1 & 44.9 & 0.1 & 45.4 & 0.1 & 41.3 & 0.1 & 42.2 & 0.1 & 39.4 & 0.1 & 40.1 & 0.1 & 37.6 & 0.1 & 37.8 & 0.1 \\
\hline 54L3 & 47.8 & 0.1 & 47.9 & 0.1 & 44.7 & 0.1 & 44.7 & 0.1 & 42.9 & 0.1 & 42.6 & 0.1 & 41.3 & 0.1 & 40.2 & 0.1 \\
\hline $55 \mathrm{~L} 1$ & 46.4 & 0.1 & 46.9 & 0.1 & 43.4 & 0.1 & 43.6 & 0.1 & 40.9 & 0. & 41.5 & 0.1 & 38.2 & 0.1 & 39.1 & 0.1 \\
\hline 55L3 & 49.2 & 0.1 & 49.0 & 0.1 & 46.0 & 0.1 & 45.4 & 0.1 & 44.6 & 0.1 & 43.4 & 0.1 & 42.0 & 0.1 & 40.8 & 0.1 \\
\hline $56 \mathrm{~L} 1$ & 48.1 & 0.1 & 48.6 & 0.1 & 44.7 & 0.1 & 45.4 & 0.1 & 42.8 & 0.1 & 43.6 & 0.1 & 40.1 & 0.1 & 41.0 & 0.1 \\
\hline $56 \mathrm{~L} 3$ & 50.0 & 0.1 & 49.1 & 0.1 & 47.3 & 0.1 & 45.7 & 0.1 & 45.2 & 0.1 & 43.6 & 0.1 & 43.2 & 0.1 & 40.5 & 0.1 \\
\hline 65L1 & 49.8 & 0.1 & 50.5 & 0.1 & 46.7 & 0.1 & 47.4 & 0.1 & 44.4 & 0.1 & 45.4 & 0.1 & 42.0 & 0.1 & 42.7 & 0.1 \\
\hline $65 \mathrm{~L} 3$ & 52.1 & 0.1 & 50.7 & 0.1 & 49.0 & 0.1 & 46.9 & 0.1 & 47.4 & 0.1 & 44.7 & 0.1 & 45.8 & 0.1 & 41.6 & 0.1 \\
\hline 66L1 & 51.4 & 0.2 & 51.9 & 0.2 & 47.6 & 0.2 & 48.3 & 0.2 & 45.6 & 0.2 & 46.1 & 0.2 & 43.4 & 0.1 & 43.8 & 0.1 \\
\hline $66 \mathrm{~L} 3$ & 53.9 & 0.1 & 50.8 & 0.1 & 50.4 & 0.1 & 46.5 & 0.1 & 49.2 & 0.1 & 44.1 & 0.1 & 47.1 & 0.1 & 40.9 & 0.1 \\
\hline
\end{tabular}

SE, standard error;

${ }^{a}$ indicates that the quality of the data is not sufficient to allow an accurate determination, or that the stability is too low to determine $T_{1 / 2}$. 
Table S1 $T_{1 / 2}$ obtained from UV melting and annealing processes at different pHs. (Continued_03)

\begin{tabular}{|c|c|c|c|c|c|c|c|c|c|c|c|c|c|c|c|c|}
\hline \multirow{3}{*}{$\begin{array}{c}\mathrm{pH} \\
\text { Oligo }\end{array}$} & \multicolumn{4}{|c|}{ pH 6.40} & \multicolumn{4}{|c|}{ pH 6.51} & \multicolumn{4}{|c|}{ pH 6.61} & \multicolumn{4}{|c|}{$\mathrm{pH} 6.70$} \\
\hline & \multicolumn{2}{|c|}{ heating } & \multicolumn{2}{|c|}{ cooling } & \multicolumn{2}{|c|}{ heating } & \multicolumn{2}{|c|}{ cooling } & \multicolumn{2}{|c|}{ heating } & \multicolumn{2}{|c|}{ cooling } & \multicolumn{2}{|c|}{ heating } & \multicolumn{2}{|c|}{ cooling } \\
\hline & $\begin{array}{c}\mathrm{T}_{1 / 2}, \\
{ }^{\circ} \mathrm{C}\end{array}$ & $\begin{array}{l}\mathrm{SE}, \\
{ }^{\circ} \mathrm{C}\end{array}$ & $\begin{array}{l}\mathrm{T}_{1 / 2} \\
{ }^{\circ} \mathrm{C}\end{array}$ & $\begin{array}{c}\text { SE, } \\
{ }^{\circ} \mathrm{C}\end{array}$ & $\begin{array}{l}\mathrm{T}_{1 / 2} \\
{ }^{\circ} \mathrm{C}\end{array}$ & $\begin{array}{l}\text { SE, } \\
{ }^{\circ} \mathrm{C}\end{array}$ & $\begin{array}{c}\mathrm{T}_{1 / 2}, \\
{ }^{\circ} \mathrm{C}\end{array}$ & $\begin{array}{l}\text { SE, } \\
{ }^{\circ} \mathrm{C}\end{array}$ & $\begin{array}{c}\mathrm{T}_{1 / 2}, \\
{ }^{\circ} \mathrm{C}\end{array}$ & $\begin{array}{l}\text { SE, } \\
{ }^{\circ} \mathrm{C}\end{array}$ & $\begin{array}{c}\mathrm{T}_{1 / 2} \\
{ }^{\circ} \mathrm{C}\end{array}$ & $\begin{array}{l}\mathrm{SE}, \\
{ }^{\circ} \mathrm{C}\end{array}$ & $\begin{array}{l}\mathrm{T}_{1 / 2} \\
{ }^{\circ} \mathrm{C}\end{array}$ & $\begin{array}{l}\text { SE, } \\
{ }^{\circ} \mathrm{C}\end{array}$ & $\begin{array}{c}\mathrm{T}_{1 / 2} \\
{ }^{\circ} \mathrm{C}\end{array}$ & $\begin{array}{l}\text { SE, } \\
{ }^{\circ} \mathrm{C}\end{array}$ \\
\hline 33L1 & 25.4 & 0.2 & 14.9 & 0.2 & 23.4 & 0.4 & 12.4 & 0.2 & 21.6 & 0.4 & 9.1 & 0.3 & a & a & a & a \\
\hline 33L3 & 26.5 & 0.1 & 26.3 & 0.1 & 23.4 & 0.1 & 24.0 & 0.1 & 20.1 & 0.1 & 21.4 & 0.1 & 19.0 & 0.1 & 19.5 & 0.1 \\
\hline 34L1 & 23.5 & 0.3 & 20.5 & 0.2 & 22.0 & 0.6 & 17.8 & 0.2 & 19.2 & 0.4 & 14.9 & 0.2 & 17.3 & 0.2 & 12.9 & 0.2 \\
\hline 34L3 & 28.6 & 0.1 & 28.6 & 0.1 & 25.6 & 0.1 & 26.0 & 0.1 & 23.2 & 0.1 & 23.5 & 0.1 & 21.0 & 0.1 & 21.3 & 0.1 \\
\hline 43L1 & 27.4 & 0.1 & 26.1 & 0.1 & 22.7 & 0.3 & 23.1 & 0.2 & 20.9 & 0.2 & 20.7 & 0.1 & 8.0 & 0.2 & 18.5 & 0.1 \\
\hline 43L3 & 32.5 & 0.1 & 32.7 & 0.1 & 31.3 & 0.1 & 29.8 & 0.1 & 27.7 & 0.1 & 27.7 & 0.1 & 25.7 & 0.1 & 25.8 & 0.1 \\
\hline 44L1 & 29.1 & 0.1 & 28.0 & 0.1 & 25.5 & 0.1 & 25.3 & 0.1 & 22.6 & 0.1 & 23.0 & 0.1 & 20.8 & 0.2 & 20.8 & 0.1 \\
\hline 44L3 & 34.8 & 0.1 & 34.3 & 0.1 & 31.2 & 0.1 & 31.7 & 0.1 & 29.2 & 0.1 & 29.1 & 0.1 & 27.2 & 0.1 & 26.7 & 0.1 \\
\hline 45L1 & 32.3 & 0.1 & 32.0 & 0.1 & 28.0 & 0.1 & 29.8 & 0.1 & 26.1 & 0.1 & 26.8 & 0.1 & 24.3 & 0.1 & 24.4 & 0.1 \\
\hline $45 \mathrm{~L} 3$ & 36.6 & 0.1 & 35.3 & 0.1 & 32.4 & 0.1 & 32.9 & 0.1 & 31.1 & 0.1 & 30.0 & 0.1 & 29.6 & 0.1 & 27.2 & 0.1 \\
\hline 54L1 & 33.6 & 0.1 & 34.9 & 0.1 & 30.9 & 0.1 & 32.5 & 0.1 & 29.1 & 0.1 & 29.6 & 0.1 & 27.3 & 0.1 & 27.0 & 0.1 \\
\hline $54 \mathrm{~L} 3$ & 37.5 & 0.1 & 37.4 & 0.1 & 35.2 & 0.1 & 34.9 & 0.1 & 34.3 & 0.1 & 31.9 & 0.1 & 33.1 & 0.1 & 29.1 & 0.1 \\
\hline $55 \mathrm{~L} 1$ & 35.0 & 0.1 & 36.2 & 0.1 & 32.2 & 0.1 & 33.7 & 0.1 & 30.4 & 0.1 & 31.0 & 0.1 & 28.6 & 0.1 & 28.5 & 0.1 \\
\hline $55 \mathrm{~L} 3$ & 40.3 & 0.1 & 38.0 & 0.1 & 37.9 & 0.1 & 34.6 & 0.1 & 35.7 & 0.1 & 31.5 & 0.1 & 34.5 & 0.1 & 28.5 & 0.1 \\
\hline $56 \mathrm{~L} 1$ & 36.8 & 0.1 & 37.9 & 0.1 & 35.1 & 0.1 & 35.5 & 0.1 & 32.5 & 0.1 & 32.8 & 0.1 & 30.5 & 0.1 & 30.3 & 0.1 \\
\hline $56 \mathrm{L3}$ & 41.1 & 0.1 & 37.6 & 0.1 & 38.9 & 0.1 & 34.1 & 0.1 & 37.0 & 0.1 & 30.7 & 0.1 & 35.4 & 0.1 & 27.5 & 0.1 \\
\hline 65L1 & 39.4 & 0.1 & 39.7 & 0.1 & 37.0 & 0.1 & 37.1 & 0.1 & 34.4 & 0.1 & 34.5 & 0.1 & 32.6 & 0.1 & 31.9 & 0.1 \\
\hline $65 \mathrm{~L} 3$ & 43.5 & 0.1 & 38.4 & 0.1 & 41.3 & 0.2 & 34.7 & 0.1 & 39.8 & 0.1 & 31.1 & 0.1 & 38.1 & 0.1 & 28.1 & 0.1 \\
\hline 66L1 & 40.8 & 0.1 & 40.9 & 0.1 & 37.6 & 0.1 & 38.0 & 0.1 & 35.5 & 0.1 & 35.4 & 0.1 & 33.1 & 0.1 & 32.9 & 0.1 \\
\hline $66 \mathrm{L3}$ & 44.3 & 0.1 & 37.2 & 0.1 & 42.6 & 0.1 & 33.7 & 0.1 & 41.0 & 0.1 & 30.2 & 0.1 & 38.8 & 0.2 & 27.4 & 0.1 \\
\hline
\end{tabular}

SE, standard error;

${ }^{a}$ indicates that the quality of the data is not sufficient to allow an accurate determination, or that the stability is too low to determine $T_{1 / 2}$. 
Table S1 $T_{1 / 2}$ obtained from UV melting and annealing processes at different pHs. (Continued_04)

\begin{tabular}{|c|c|c|c|c|c|c|c|c|c|c|c|c|c|c|c|c|}
\hline \multirow{3}{*}{$\begin{array}{c}\mathrm{pH} \\
\text { Oligo }\end{array}$} & \multicolumn{4}{|c|}{ pH 6.80} & \multicolumn{4}{|c|}{ pH 6.90} & \multicolumn{4}{|c|}{ pH 7.01} & \multicolumn{4}{|c|}{ pH 7.10} \\
\hline & \multicolumn{2}{|c|}{ heating } & \multicolumn{2}{|c|}{ cooling } & \multicolumn{2}{|c|}{ heating } & \multicolumn{2}{|c|}{ cooling } & \multicolumn{2}{|c|}{ heating } & \multicolumn{2}{|c|}{ cooling } & \multicolumn{2}{|c|}{ heating } & \multicolumn{2}{|c|}{ cooling } \\
\hline & $\begin{array}{l}\mathrm{T}_{1 / 2} \\
{ }^{\circ} \mathrm{C}\end{array}$ & $\begin{array}{l}\text { SE, } \\
{ }^{\circ} \mathrm{C}\end{array}$ & $\begin{array}{c}\mathrm{T}_{1 / 2} \\
{ }^{\circ} \mathrm{C}\end{array}$ & $\begin{array}{l}\text { SE, } \\
{ }^{\circ} \mathrm{C}\end{array}$ & $\begin{array}{c}\mathrm{T}_{1 / 2} \\
{ }^{\circ} \mathrm{C}\end{array}$ & $\begin{array}{l}\text { SE, } \\
{ }^{\circ} \mathrm{C}\end{array}$ & $\begin{array}{c}\mathrm{T}_{1 / 2}, \\
{ }^{\circ} \mathrm{C}\end{array}$ & $\begin{array}{l}\text { SE, } \\
{ }^{\circ} \mathrm{C}\end{array}$ & $\begin{array}{c}\mathrm{T}_{1 / 2} \\
{ }^{\circ} \mathrm{C}\end{array}$ & $\begin{array}{l}\text { SE, } \\
{ }^{\circ} \mathrm{C}\end{array}$ & $\begin{array}{c}\mathrm{T}_{1 / 2} \\
{ }^{\circ} \mathrm{C}\end{array}$ & $\begin{array}{l}\text { SE, } \\
{ }^{\circ} \mathrm{C}\end{array}$ & $\begin{array}{c}\mathrm{T}_{1 / 2} \\
{ }^{\circ} \mathrm{C}\end{array}$ & $\begin{array}{l}\text { SE, } \\
{ }^{\circ} \mathrm{C}\end{array}$ & $\begin{array}{c}\mathrm{T}_{1 / 2} \\
{ }^{\circ} \mathrm{C}\end{array}$ & $\begin{array}{l}\text { SE, } \\
{ }^{\circ} \mathrm{C}\end{array}$ \\
\hline 33L1 & a & a & a & a & a & a & a & a & a & a & a & a & a & a & a & a \\
\hline 33L3 & 17.2 & 0.1 & 17.5 & 0.1 & 14.9 & 0.2 & 15.3 & 0.2 & 12.8 & 0.1 & 13.1 & 0.1 & 10.7 & 0.1 & 11.1 & 0.1 \\
\hline 34L1 & $a$ & $a$ & $a$ & $a$ & a & a & $a$ & a & a & a & $a$ & $a$ & $a$ & $a$ & a & a \\
\hline 34L3 & 19.1 & 0.1 & 19.5 & 0.2 & 16.6 & 0.2 & 17.0 & 0.2 & 14.7 & 0.1 & 14.8 & 0.1 & 12.4 & 0.1 & 12.3 & 0.1 \\
\hline 43L1 & 16.1 & 0.2 & 16.3 & 0.2 & 13.3 & 0.3 & 13.8 & 0.3 & 11.4 & 0.1 & 11.7 & 0.1 & 9.2 & 0.2 & 9.5 & 0.2 \\
\hline $43 L 3$ & 24.0 & 0.1 & 23.8 & 0.1 & 21.6 & 0.1 & 21.3 & 0.1 & 19.4 & 0.1 & 19.2 & 0.1 & 18.5 & 0.1 & 16.3 & 0.1 \\
\hline 44L1 & 18.0 & 0.2 & 18.6 & 0.2 & 15.5 & 0.2 & 16.0 & 0.2 & 13.0 & 0.1 & 13.9 & 0.1 & 10.9 & 0.2 & 11.6 & 0.1 \\
\hline 44L3 & 25.1 & 0.1 & 24.5 & 0.1 & 23.2 & 0.1 & 22.0 & 0.1 & 20.9 & 0.1 & 19.5 & 0.1 & 19.1 & 0.1 & 16.3 & 0.1 \\
\hline $45 \mathrm{~L} 1$ & 21.5 & 0.1 & 22.3 & 0.1 & 19.3 & 0.1 & 19.7 & 0.1 & 16.5 & 0.1 & 17.3 & 0.1 & 14.4 & 0.1 & 14.6 & 0.1 \\
\hline $45 \mathrm{~L} 3$ & 27.2 & 0.1 & 25.1 & 0.1 & 25.4 & 0.1 & 22.2 & 0.1 & 23.5 & 0.1 & 19.6 & 0.1 & 21.6 & 0.1 & 16.7 & 0.1 \\
\hline 54L1 & 24.6 & 0.1 & 25.1 & 0.1 & 22.6 & 0.1 & 22.3 & 0.1 & 20.1 & 0.1 & 20.3 & 0.1 & 17.9 & 0.1 & 17.4 & 0.1 \\
\hline 54L3 & 30.5 & 0.1 & 26.8 & 0.1 & 29.3 & 0.1 & 23.9 & 0.1 & 27.7 & 0.1 & 21.2 & 0.1 & 25.4 & 0.1 & 18.2 & 0.1 \\
\hline $55 \mathrm{~L} 1$ & 26.1 & 0.1 & 26.4 & 0.1 & 23.6 & 0.1 & 24.0 & 0.1 & 21.3 & 0.1 & 21.5 & 0.1 & 19.0 & 0.1 & 18.8 & 0.1 \\
\hline 55L3 & 32.4 & 0.1 & 25.9 & 0.1 & 30.6 & 0.1 & 23.2 & 0.1 & 28.8 & 0.1 & 20.0 & 0.1 & 27.1 & 0.1 & 16.8 & 0.1 \\
\hline $56 \mathrm{~L} 1$ & 27.9 & 0.1 & 28.3 & 0.1 & 26.1 & 0.1 & 25.1 & 0.1 & 23.0 & 0.1 & 23.4 & 0.1 & 21.2 & 0.1 & 20.4 & 0.1 \\
\hline $56 \mathrm{~L} 3$ & 33.5 & 0.1 & 25.0 & 0.1 & 32.0 & 0.1 & 21.4 & 0.1 & 30.1 & 0.1 & 19.0 & 0.1 & 27.8 & 0.1 & 16.2 & 0.1 \\
\hline 65L1 & 30.1 & 0.1 & 29.7 & 0.1 & 28.3 & 0.1 & 26.7 & 0.1 & 25.4 & 0.1 & 24.6 & 0.1 & 23.2 & 0.1 & 22.2 & 0.1 \\
\hline $65 \mathrm{~L} 3$ & 36.3 & 0.1 & 25.4 & 0.1 & 34.7 & 0.1 & 21.7 & 0.1 & 33.3 & 0.1 & 19.2 & 0.1 & 30.7 & 0.1 & 16.6 & 0.1 \\
\hline 66L1 & 31.2 & 0.1 & 30.4 & 0.1 & 29.3 & 0.1 & 27.4 & 0.1 & 26.6 & 0.1 & 25.1 & 0.1 & 25.1 & 0.1 & 22.2 & 0.1 \\
\hline $66 \mathrm{~L} 3$ & 37.4 & 0.1 & 24.4 & 0.1 & 35.7 & 0.1 & 21.0 & 0.1 & 33.4 & 0.1 & 18.4 & 0.1 & 31.4 & 0.1 & 16.2 & 0.1 \\
\hline
\end{tabular}

SE, standard error;

${ }^{a}$ indicates that the quality of the data is not sufficient to allow an accurate determination, or that the stability is too low to determine $T_{1 / 2}$. 
Table S1 $T_{1 / 2}$ obtained from UV melting and annealing processes at different pHs. (Continued_05)

\begin{tabular}{|c|c|c|c|c|c|c|c|c|c|c|c|c|c|c|c|c|}
\hline \multirow{3}{*}{$\begin{array}{c}\mathrm{pH} \\
\text { Oligo }\end{array}$} & \multicolumn{4}{|c|}{ pH 7.21 } & \multicolumn{4}{|c|}{$\mathrm{pH} 7.30$} & \multicolumn{4}{|c|}{$\mathrm{pH} 7.40$} & \multicolumn{4}{|c|}{$\mathrm{pH} 7.50$} \\
\hline & \multicolumn{2}{|c|}{ heating } & \multicolumn{2}{|c|}{ cooling } & \multicolumn{2}{|c|}{ heating } & \multicolumn{2}{|c|}{ cooling } & \multicolumn{2}{|c|}{ heating } & \multicolumn{2}{|c|}{ cooling } & \multicolumn{2}{|c|}{ heating } & \multicolumn{2}{|c|}{ cooling } \\
\hline & $\begin{array}{c}\mathrm{T}_{1 / 2} \\
{ }^{\circ} \mathrm{C}\end{array}$ & $\begin{array}{l}\mathrm{SE}, \\
{ }^{\circ} \mathrm{C}\end{array}$ & $\begin{array}{c}\mathrm{T}_{1 / 2} \\
{ }^{\circ} \mathrm{C}\end{array}$ & $\begin{array}{c}\text { SE, } \\
{ }^{\circ} \mathrm{C}\end{array}$ & $\begin{array}{c}\mathrm{T}_{1 / 2} \\
{ }^{\circ} \mathrm{C}\end{array}$ & $\begin{array}{c}\text { SE, } \\
{ }^{\circ} \mathrm{C}\end{array}$ & $\begin{array}{c}\mathrm{T}_{1 / 2}, \\
{ }^{\circ} \mathrm{C}\end{array}$ & $\begin{array}{c}\mathrm{SE}, \\
{ }^{\circ} \mathrm{C}\end{array}$ & $\begin{array}{c}\mathrm{T}_{1 / 2} \\
{ }^{\circ} \mathrm{C}\end{array}$ & $\begin{array}{l}\text { SE, } \\
{ }^{\circ} \mathrm{C}\end{array}$ & $\begin{array}{c}\mathrm{T}_{1 / 2} \\
{ }^{\circ} \mathrm{C}\end{array}$ & $\begin{array}{c}\mathrm{SE}, \\
{ }^{\circ} \mathrm{C}\end{array}$ & $\begin{array}{c}\mathrm{T}_{1 / 2}, \\
{ }^{\circ} \mathrm{C}\end{array}$ & $\begin{array}{c}\mathrm{SE}, \\
{ }^{\circ} \mathrm{C}\end{array}$ & $\begin{array}{c}\mathrm{T}_{1 / 2}, \\
{ }^{\circ} \mathrm{C}\end{array}$ & $\begin{array}{c}\mathrm{SE}, \\
{ }^{\circ} \mathrm{C}\end{array}$ \\
\hline 33L1 & $a$ & $a$ & $a$ & $a$ & $a$ & a & $a$ & $a$ & $a$ & $a$ & $a$ & $a$ & $a$ & $a$ & $a$ & $a$ \\
\hline $33 L 3$ & a & a & a & a & $a$ & a & a & a & a & a & a & $a$ & a & $a$ & a & $a$ \\
\hline 34L1 & a & $a$ & a & $a$ & a & a & $a$ & a & a & $a$ & $a$ & $a$ & a & $a$ & $a$ & $a$ \\
\hline 34L3 & a & a & a & $a$ & a & $a$ & $a$ & a & a & a & a & $a$ & a & $a$ & $a$ & $a$ \\
\hline 43L1 & $a$ & $a$ & $a$ & $a$ & $a$ & $a$ & a & a & $a$ & a & $a$ & $a$ & $a$ & $a$ & a & $a$ \\
\hline 43L3 & 16.1 & 0.1 & 13.7 & 0.1 & 14.6 & 0.1 & 11.6 & 0.1 & 12.4 & 0.2 & $a$ & $a$ & 11.0 & 0.1 & $a$ & $a$ \\
\hline 44L1 & $a$ & $a$ & $a$ & a & $a$ & $a$ & $a$ & $a$ & $a$ & a & $a$ & $a$ & $a$ & $a$ & a & $a$ \\
\hline 44L3 & 16.7 & 0.1 & 14.2 & 0.1 & 15.1 & 0.1 & 12.2 & 0.1 & 12.9 & 0.1 & a & a & 11.5 & 0.1 & a & a \\
\hline 45L1 & 11.7 & 0.1 & 12.4 & 0.1 & 9.9 & 0.1 & 10.7 & $a$ & a & a & $a$ & $a$ & a & $a$ & $a$ & $a$ \\
\hline $45 \mathrm{~L} 3$ & 19.2 & 0.1 & 14.1 & 0.1 & 17.7 & 0.1 & 11.8 & 0.1 & 15.1 & 0.1 & $a$ & $a$ & 14.3 & 0.1 & $a$ & $a$ \\
\hline 54L1 & 15.3 & 0.1 & 15.0 & 0.1 & 13.4 & 0.1 & 13.2 & 0.1 & 10.9 & 0.2 & 10.4 & 0.1 & $a$ & a & a & $a$ \\
\hline $54 \mathrm{~L} 3$ & 23.4 & 0.1 & 15.4 & 0.1 & 22.2 & 0.1 & 12.7 & 0.1 & 19.6 & 0.1 & 9.5 & 0.3 & 18.6 & 0.1 & a & $a$ \\
\hline 55L1 & 16.4 & 0.1 & 16.6 & 0.1 & 14.5 & 0.1 & 14.5 & 0.1 & 12.1 & 0.1 & 11.9 & 0.1 & a & $a$ & $a$ & $a$ \\
\hline $55 \mathrm{~L} 3$ & 24.9 & 0.1 & 14.2 & 0.1 & 23.4 & 0.1 & 11.0 & 0.2 & 21.1 & 0.1 & a & $a$ & 19.9 & 0.1 & $a$ & $a$ \\
\hline $56 \mathrm{~L} 1$ & 18.6 & 0.1 & 18.1 & 0.1 & 16.9 & 0.1 & 16.0 & 0.1 & 14.3 & 0.1 & 12.4 & 0.1 & 12.4 & 0.1 & 9.7 & 0.1 \\
\hline $56 \mathrm{~L} 3$ & 25.8 & 0.1 & 12.9 & 0.1 & 24.1 & 0.1 & 10.6 & 0.2 & 21.8 & 0.1 & $a$ & $a$ & 20.3 & 0.1 & $a$ & $a$ \\
\hline 65L1 & 21.0 & 0.1 & 19.0 & 0.1 & 19.1 & 0.1 & 17.1 & 0.1 & 16.9 & 0.1 & 13.7 & 0.1 & 14.9 & 0.1 & 10.7 & 0.1 \\
\hline $65 \mathrm{~L} 3$ & 29.2 & 0.1 & 12.9 & 0.1 & 27.3 & 0.1 & 10.7 & 0.1 & 25.3 & 0.1 & $a$ & $a$ & 24.1 & 0.1 & $a$ & $a$ \\
\hline 66L1 & 22.7 & 0.1 & 19.0 & 0.1 & 20.5 & 0.1 & 17.3 & 0.1 & 18.6 & 0.1 & 13.6 & 0.1 & 16.8 & 0.1 & 11.0 & 0.1 \\
\hline $66 \mathrm{~L} 3$ & 29.8 & 0.1 & 12.2 & 0.1 & 28.1 & 0.1 & 10.6 & 0.1 & 26.1 & 0.1 & a & a & 24.6 & 0.1 & a & a \\
\hline
\end{tabular}

SE, standard error;

${ }^{a}$ indicates that the quality of the data is not sufficient to allow an accurate determination, or that the stability is too low to determine $T_{1 / 2}$. 
Table S1 $T_{1 / 2}$ obtained from UV melting and annealing processes at different pHs. (Continued_06)

\begin{tabular}{ccccccccc}
\hline $\mathrm{pH}$ & \multicolumn{4}{c}{$\mathrm{pH} 7.72$} & \multicolumn{5}{c}{$\mathrm{pH} 7.98$} \\
\hline & \multicolumn{2}{c}{ heating } & \multicolumn{2}{c}{ cooling } & \multicolumn{2}{c}{ heating } & \multicolumn{2}{c}{ cooling } \\
\cline { 2 - 9 } Oligo & $\mathrm{T}_{1 / 2}$, & $\mathrm{SE}$, & $\mathrm{T}_{1 / 2}$, & $\mathrm{SE}$, & $\mathrm{T}_{1 / 2}$, & $\mathrm{SE}$, & $\mathrm{T}_{1 / 2}$, & $\mathrm{SE}$, \\
& ${ }^{\circ} \mathrm{C}$ & ${ }^{\circ} \mathrm{C}$ & ${ }^{\circ} \mathrm{C}$ & ${ }^{\circ} \mathrm{C}$ & ${ }^{\circ} \mathrm{C}$ & ${ }^{\circ} \mathrm{C}$ & ${ }^{\circ} \mathrm{C}$ & ${ }^{\circ} \mathrm{C}$ \\
\hline 33L1 & $\mathrm{a}$ & $\mathrm{a}$ & $\mathrm{a}$ & $\mathrm{a}$ & $\mathrm{a}$ & $\mathrm{a}$ & $\mathrm{a}$ & $\mathrm{a}$ \\
33L3 & $\mathrm{a}$ & $\mathrm{a}$ & $\mathrm{a}$ & $\mathrm{a}$ & $\mathrm{a}$ & $\mathrm{a}$ & $\mathrm{a}$ & $\mathrm{a}$ \\
34L1 & $\mathrm{a}$ & $\mathrm{a}$ & $\mathrm{a}$ & $\mathrm{a}$ & $\mathrm{a}$ & $\mathrm{a}$ & $\mathrm{a}$ & $\mathrm{a}$ \\
34L3 & $\mathrm{a}$ & $\mathrm{a}$ & $\mathrm{a}$ & $\mathrm{a}$ & $\mathrm{a}$ & $\mathrm{a}$ & $\mathrm{a}$ & $\mathrm{a}$ \\
43L1 & $\mathrm{a}$ & $\mathrm{a}$ & $\mathrm{a}$ & $\mathrm{a}$ & $\mathrm{a}$ & $\mathrm{a}$ & $\mathrm{a}$ & $\mathrm{a}$ \\
43L3 & $\mathrm{a}$ & $\mathrm{a}$ & $\mathrm{a}$ & $\mathrm{a}$ & $\mathrm{a}$ & $\mathrm{a}$ & $\mathrm{a}$ & $\mathrm{a}$ \\
44L1 & $\mathrm{a}$ & $\mathrm{a}$ & $\mathrm{a}$ & $\mathrm{a}$ & $\mathrm{a}$ & $\mathrm{a}$ & $\mathrm{a}$ & $\mathrm{a}$ \\
44L3 & 9.7 & 0.1 & $\mathrm{a}$ & $\mathrm{a}$ & $\mathrm{a}$ & $\mathrm{a}$ & $\mathrm{a}$ & $\mathrm{a}$ \\
45L1 & $\mathrm{a}$ & $\mathrm{a}$ & $\mathrm{a}$ & $\mathrm{a}$ & $\mathrm{a}$ & $\mathrm{a}$ & $\mathrm{a}$ & $\mathrm{a}$ \\
45L3 & 11.8 & 0.2 & $\mathrm{a}$ & $\mathrm{a}$ & $\mathrm{a}$ & $\mathrm{a}$ & $\mathrm{a}$ & $\mathrm{a}$ \\
$54 \mathrm{~L} 1$ & $\mathrm{a}$ & $\mathrm{a}$ & $\mathrm{a}$ & $\mathrm{a}$ & $\mathrm{a}$ & $\mathrm{a}$ & $\mathrm{a}$ & $\mathrm{a}$ \\
$54 \mathrm{~L} 3$ & 16.8 & 0.2 & $\mathrm{a}$ & $\mathrm{a}$ & $\mathrm{a}$ & $\mathrm{a}$ & $\mathrm{a}$ & $\mathrm{a}$ \\
$55 \mathrm{~L} 1$ & $\mathrm{a}$ & $\mathrm{a}$ & $\mathrm{a}$ & $\mathrm{a}$ & $\mathrm{a}$ & $\mathrm{a}$ & $\mathrm{a}$ & $\mathrm{a}$ \\
$55 \mathrm{~L} 3$ & 18.3 & 0.3 & $\mathrm{a}$ & $\mathrm{a}$ & $\mathrm{a}$ & $\mathrm{a}$ & $\mathrm{a}$ & $\mathrm{a}$ \\
$56 \mathrm{~L} 1$ & 10.0 & 0.1 & $\mathrm{a}$ & $\mathrm{a}$ & $\mathrm{a}$ & $\mathrm{a}$ & $\mathrm{a}$ & $\mathrm{a}$ \\
$56 \mathrm{~L} 3$ & 12.2 & 0.1 & 7.4 & 0.2 & $\mathrm{a}$ & $\mathrm{a}$ & $\mathrm{a}$ & $\mathrm{a}$ \\
$65 \mathrm{~L} 1$ & 12.2 & 0.1 & 7.4 & 0.2 & $\mathrm{a}$ & $\mathrm{a}$ & $\mathrm{a}$ & $\mathrm{a}$ \\
$65 \mathrm{~L} 3$ & 21.8 & 0.2 & $\mathrm{a}$ & $\mathrm{a}$ & $\mathrm{a}$ & $\mathrm{a}$ & $\mathrm{a}$ & $\mathrm{a}$ \\
$66 \mathrm{~L} 1$ & 13.8 & 0.1 & 6.0 & 0.5 & $\mathrm{a}$ & $\mathrm{a}$ & $\mathrm{a}$ & $\mathrm{a}$ \\
$66 \mathrm{~L} 3$ & 21.4 & 0.3 & $\mathrm{a}$ & $\mathrm{a}$ & $\mathrm{a}$ & $\mathrm{a}$ & $\mathrm{a}$ & $\mathrm{a}$ \\
\hline & & & & & & & &
\end{tabular}

SE, standard error;

${ }^{a}$ indicates that the quality of the data is not sufficient to allow an accurate determination, or that the stability is too low to determine $T_{1 / 2}$. 
Table S2 Linear fitting results of $T_{1 / 2}$ as a function of $\mathrm{pH}$, and $\mathrm{pH}_{T}$ as a function of temperature. ${ }^{a}$

\begin{tabular}{|c|c|c|c|c|c|c|}
\hline \multirow{2}{*}{ Name } & \multicolumn{3}{|c|}{ Temperature transition } & \multicolumn{3}{|c|}{$\mathrm{pH}$ transition } \\
\hline & Slope & Intercept & Pearson's r & Slope & Intercept & Pearson's r \\
\hline 33L1 & $-22.37 \pm 0.39$ & $164.68 \pm 2.31$ & -0.99574 & $-0.032 \pm 0.002$ & $7.03 \pm 0.05$ & -0.97451 \\
\hline 33L3 & $-21.36 \pm 0.23$ & $162.74 \pm 1.42$ & -0.99780 & $-0.043 \pm 0.001$ & $7.58 \pm 0.03$ & -0.99657 \\
\hline 34L1 & $-23.38 \pm 0.67$ & $171.43 \pm 3.98$ & -0.98785 & $-0.037 \pm 0.001$ & $7.25 \pm 0.03$ & -0.99366 \\
\hline 34L3 & $-22.89 \pm 0.14$ & $174.87 \pm 0.88$ & -0.99927 & $-0.042 \pm 0.000$ & $7.62 \pm 0.01$ & -0.99908 \\
\hline 43L1 & $-23.85 \pm 0.16$ & $178.54 \pm 1.00$ & -0.99913 & $-0.039 \pm 0.001$ & $7.45 \pm 0.02$ & -0.99806 \\
\hline 43L3 & $-21.54 \pm 0.11$ & $170.36 \pm 0.70$ & -0.99943 & $-0.046 \pm 0.001$ & $7.93 \pm 0.02$ & -0.99826 \\
\hline 44L1 & $-24.45 \pm 0.12$ & $184.76 \pm 0.72$ & -0.99957 & $-0.039 \pm 0.000$ & $7.53 \pm 0.01$ & -0.99940 \\
\hline 44L3 & $-23.81 \pm 0.49$ & $186.07 \pm 3.09$ & -0.99107 & $-0.043 \pm 0.000$ & $7.90 \pm 0.02$ & -0.99882 \\
\hline 45L1 & $-24.30 \pm 0.12$ & $187.35 \pm 0.75$ & -0.99949 & $-0.039 \pm 0.000$ & $7.69 \pm 0.02$ & -0.99843 \\
\hline 45L3 & $-23.59 \pm 0.10$ & $186.68 \pm 0.63$ & -0.99962 & $-0.042 \pm 0.000$ & $7.93 \pm 0.02$ & -0.99900 \\
\hline 54L1 & $-24.60 \pm 0.14$ & $192.28 \pm 0.86$ & -0.99932 & $-0.040 \pm 0.001$ & $7.83 \pm 0.02$ & -0.99740 \\
\hline 54L3 & $-22.79 \pm 0.15$ & $183.71 \pm 0.97$ & -0.99900 & $-0.043 \pm 0.000$ & $8.06 \pm 0.02$ & -0.99929 \\
\hline 55L1 & $-24.36 \pm 0.10$ & $192.03 \pm 0.66$ & -0.99960 & $-0.040 \pm 0.000$ & $7.88 \pm 0.01$ & -0.99921 \\
\hline 55L3 & $-23.70 \pm 0.14$ & $190.44 \pm 0.71$ & -0.99952 & $-0.041 \pm 0.001$ & $8.04 \pm 0.02$ & -0.99859 \\
\hline 56L1 & $-24.42 \pm 0.10$ & $194.24 \pm 0.62$ & -0.99964 & $-0.040 \pm 0.000$ & $7.95 \pm 0.02$ & -0.99874 \\
\hline $56 \mathrm{~L} 3$ & $-24.13 \pm 0.12$ & $193.56 \pm 0.78$ & -0.99945 & $-0.041 \pm 0.001$ & $8.04 \pm 0.02$ & -0.99815 \\
\hline 65L1 & $-24.92 \pm 0.12$ & $199.48 \pm 0.77$ & -0.99946 & $-0.038 \pm 0.001$ & $7.95 \pm 0.03$ & -0.99589 \\
\hline 65L3 & $-24.33 \pm 0.13$ & $196.52 \pm 0.83$ & -0.99937 & $-0.041 \pm 0.001$ & $8.16 \pm 0.03$ & -0.99710 \\
\hline 66L1 & $-25.01 \pm 0.15$ & $200.98 \pm 0.93$ & -0.99921 & $-0.038 \pm 0.000$ & $8.00 \pm 0.02$ & -0.99837 \\
\hline 66L3 & $-24.86 \pm 0.14$ & $200.22 \pm 0.85$ & -0.99937 & $-0.042 \pm 0.001$ & $8.20 \pm 0.03$ & -0.99789 \\
\hline DAP & $-23.38 \pm 0.19$ & $190.72 \pm 1.24$ & -0.99922 & $-0.042 \pm 0.001$ & $8.14 \pm 0.04$ & -0.99724 \\
\hline PDGF-A & $-24.37 \pm 0.26$ & $196.59 \pm 1.64$ & -0.99874 & $-0.040 \pm 0.001$ & $7.96 \pm 0.05$ & -0.99679 \\
\hline KRAS & $-20.60 \pm 0.24$ & $166.75 \pm 1.54$ & -0.99854 & $-0.040 \pm 0.001$ & $7.72 \pm 0.06$ & -0.99617 \\
\hline HIF-1 $1 \alpha$ & $-19.86 \pm 0.11$ & $162.14 \pm 0.69$ & -0.99967 & $-0.044 \pm 0.000$ & $7.90 \pm 0.01$ & -0.99979 \\
\hline BCL2 & $-24.17 \pm 0.12$ & $182.85 \pm 0.74$ & -0.99978 & $-0.037 \pm 0.001$ & $7.39 \pm 0.02$ & -0.99894 \\
\hline
\end{tabular}

${ }^{a}$ Linear fitting results for temperature and $\mathrm{pH}$ transitions obtained from Figures 5B, 6B, 10A-B in the main manuscript, respectively. 
Table S3 $\mathrm{pH}_{T}$ obtained from UV melting and annealing processes at different temperatures.

\begin{tabular}{|c|c|c|c|c|c|c|c|c|c|c|c|c|}
\hline \multirow[t]{3}{*}{1 emperature / $\mathrm{C}$} & \multicolumn{4}{|c|}{5} & \multicolumn{4}{|c|}{10} & \multicolumn{4}{|c|}{15} \\
\hline & \multicolumn{2}{|c|}{ heating } & \multicolumn{2}{|c|}{ cooling } & \multicolumn{2}{|c|}{ heating } & \multicolumn{2}{|c|}{ cooling } & \multicolumn{2}{|c|}{ heating } & \multicolumn{2}{|c|}{ cooling } \\
\hline & $\mathrm{pH}_{\mathrm{T}}$ & SE & $\mathrm{pH}_{\mathrm{T}}$ & SE & $\mathrm{pH}_{\mathrm{T}}$ & SE & $\mathrm{pH}_{\mathrm{T}}$ & SE & $\mathrm{pH}_{\mathrm{T}}$ & SE & $\mathrm{pH}_{\mathrm{T}}$ & SE \\
\hline 33L1 & 6.84 & 0.03 & 6.76 & 0.04 & 6.78 & 0.04 & 6.58 & 0.04 & 6.73 & 0.04 & 6.42 & 0.04 \\
\hline 33L3 & 7.41 & 0.03 & 7.39 & 0.04 & 7.14 & 0.03 & 7.17 & 0.04 & 6.93 & 0.03 & 6.95 & 0.04 \\
\hline 34L1 & 7.03 & 0.03 & 7.07 & 0.05 & 6.86 & 0.04 & 6.87 & 0.04 & 6.69 & 0.03 & 6.65 & 0.03 \\
\hline 34L3 & 7.43 & 0.06 & 7.39 & 0.04 & 7.21 & 0.03 & 7.17 & 0.02 & 7.00 & 0.02 & 7.01 & 0.02 \\
\hline 43L1 & 7.27 & 0.03 & 7.29 & 0.04 & 7.08 & 0.03 & 7.07 & 0.04 & 6.83 & 0.02 & 6.86 & 0.04 \\
\hline 43L3 & a & $a$ & 7.62 & 0.06 & 7.61 & 0.06 & 7.35 & 0.02 & 7.28 & 0.03 & 7.16 & 0.02 \\
\hline 44L1 & 7.35 & 0.03 & 7.38 & 0.03 & 7.16 & 0.03 & 7.16 & 0.02 & 6.93 & 0.03 & 6.96 & 0.02 \\
\hline 44L3 & 7.83 & 0.00 & 7.66 & 0.04 & 7.62 & 0.09 & 7.39 & 0.02 & 7.32 & 0.02 & 7.21 & 0.02 \\
\hline 45L1 & 7.50 & 0.04 & 7.62 & 0.04 & 7.26 & 0.02 & 7.31 & 0.02 & 7.08 & 0.02 & 7.12 & 0.02 \\
\hline 45L3 & 7.71 & 0.00 & 7.62 & 0.04 & 7.65 & 0.00 & 7.38 & 0.03 & 7.44 & 0.04 & 7.22 & 0.03 \\
\hline $54 \mathrm{~L} 1$ & 7.71 & 0.06 & 7.67 & 0.02 & 7.44 & 0.03 & 7.44 & 0.02 & 7.24 & 0.02 & 7.25 & 0.02 \\
\hline 54L3 & a & $a$ & $a$ & $a$ & $a$ & $a$ & 7.41 & 0.02 & 7.74 & 0.11 & 7.25 & 0.02 \\
\hline 55L1 & a & a & 7.69 & 0.03 & 7.49 & 0.02 & 7.46 & 0.01 & 7.29 & 0.01 & 7.30 & 0.01 \\
\hline $55 \mathrm{~L} 3$ & a & $a$ & $a$ & $a$ & $a$ & $a$ & 7.34 & 0.03 & $a$ & $a$ & 7.18 & 0.03 \\
\hline $56 \mathrm{~L} 1$ & a & a & a & a & 7.75 & 0.13 & 7.48 & 0.03 & 7.39 & 0.02 & 7.31 & 0.02 \\
\hline $56 \mathrm{~L} 3$ & $a$ & $a$ & $a$ & $a$ & $a$ & $a$ & 7.31 & 0.02 & 7.58 & 0.04 & 7.15 & 0.02 \\
\hline 65L1 & a & $a$ & $a$ & $a$ & $a$ & $a$ & 7.56 & 0.06 & 7.51 & 0.05 & 7.36 & 0.02 \\
\hline $65 \mathrm{~L} 3$ & a & a & $a$ & a & $a$ & a & 7.31 & 0.02 & $a$ & $a$ & 7.16 & 0.02 \\
\hline 66L1 & a & $a$ & $a$ & $a$ & $a$ & $a$ & 7.58 & 0.10 & a & $a$ & 7.37 & 0.04 \\
\hline 66L3 & a & a & a & a & a & a & 7.30 & 0.03 & a & a & 7.14 & 0.03 \\
\hline
\end{tabular}

SE, standard error;

${ }^{a}$ indicates that the quality of the data is not sufficient to allow an accurate determination

${ }^{b}$, these i-motifs are not stable enough to determine $\mathrm{pH}_{T}$. 
Table S3 $\mathrm{pH}_{T}$ obtained from UV melting and annealing processes at different temperatures. (Continued_01)

\begin{tabular}{|c|c|c|c|c|c|c|c|c|c|c|c|c|}
\hline \multirow{3}{*}{$\frac{\text { Temperature } /{ }^{\circ} \mathrm{C}}{\text { Oligo }}$} & \multicolumn{4}{|c|}{20} & \multicolumn{4}{|c|}{25} & \multicolumn{4}{|c|}{30} \\
\hline & \multicolumn{2}{|c|}{ heating } & \multicolumn{2}{|c|}{ cooling } & \multicolumn{2}{|c|}{ heating } & \multicolumn{2}{|c|}{ cooling } & \multicolumn{2}{|c|}{ heating } & \multicolumn{2}{|c|}{ cooling } \\
\hline & $\mathrm{pH}_{\mathrm{T}}$ & SE & $\mathrm{pH}$ & SE & $\mathrm{pH}_{\mathrm{T}}$ & SE & $\mathrm{pH}_{\mathrm{T}}$ & SE & $\mathrm{pH}_{\mathrm{T}}$ & SE & $\mathrm{pH}$ & SE \\
\hline 33L1 & 6.65 & 0.04 & 6.24 & 0.04 & 6.56 & 0.03 & 6.06 & 0.03 & 6.40 & 0.04 & 5.91 & 0.04 \\
\hline 33L3 & 6.64 & 0.03 & 6.72 & 0.04 & 6.50 & 0.03 & 6.49 & 0.04 & 6.25 & 0.03 & 6.31 & 0.03 \\
\hline 34L1 & 6.53 & 0.03 & 6.47 & 0.03 & 6.48 & 0.03 & 6.28 & 0.04 & 6.36 & 0.06 & 6.11 & 0.04 \\
\hline 34L3 & 6.76 & 0.02 & 6.80 & 0.02 & 6.56 & 0.02 & 6.57 & 0.02 & 6.34 & 0.01 & 6.38 & 0.02 \\
\hline 43L1 & 6.65 & 0.02 & 6.67 & 0.03 & 6.49 & 0.02 & 6.48 & 0.03 & 6.30 & 0.02 & 6.30 & 0.03 \\
\hline 43L3 & 7.02 & 0.03 & 6.98 & 0.02 & 6.75 & 0.02 & 6.76 & 0.02 & 6.58 & 0.01 & 6.53 & 0.02 \\
\hline 44L1 & 6.74 & 0.02 & 6.73 & 0.02 & 6.55 & 0.02 & 6.55 & 0.01 & 6.41 & 0.03 & 6.36 & 0.02 \\
\hline 44L3 & 7.07 & 0.02 & 7.01 & 0.02 & 6.83 & 0.02 & 6.78 & 0.02 & 6.61 & 0.00 & 6.60 & 0.01 \\
\hline 45L1 & 6.86 & 0.02 & 6.91 & 0.02 & 6.67 & 0.02 & 6.69 & 0.01 & 6.47 & 0.01 & 6.51 & 0.02 \\
\hline $45 \mathrm{~L} 3$ & 7.19 & 0.03 & 7.04 & 0.02 & 6.96 & 0.03 & 6.81 & 0.02 & 6.70 & 0.02 & 6.64 & 0.02 \\
\hline 54L1 & 7.02 & 0.02 & 7.05 & 0.02 & 6.87 & 0.00 & 6.80 & 0.02 & 6.59 & 0.02 & 6.60 & 0.01 \\
\hline $54 \mathrm{~L} 3$ & 7.38 & 0.04 & 7.07 & 0.02 & 7.14 & 0.02 & 6.89 & 0.02 & 6.89 & 0.02 & 6.68 & 0.02 \\
\hline $55 \mathrm{~L} 1$ & 7.06 & 0.02 & 7.08 & 0.01 & 6.84 & 0.02 & 6.87 & 0.01 & 6.66 & 0.01 & 6.65 & 0.01 \\
\hline 55L3 & 7.45 & 0.00 & 7.01 & 0.03 & 7.22 & 0.03 & 6.85 & 0.03 & 6.95 & 0.03 & 6.66 & 0.02 \\
\hline $56 \mathrm{~L} 1$ & 7.15 & 0.02 & 7.12 & 0.02 & 6.95 & 0.02 & 6.94 & 0.02 & 6.74 & 0.01 & 6.72 & 0.01 \\
\hline $56 \mathrm{~L} 3$ & 7.49 & 0.03 & 6.97 & 0.02 & 7.26 & 0.02 & 6.81 & 0.02 & 7.05 & 0.02 & 6.65 & 0.01 \\
\hline 65L1 & 7.25 & 0.03 & 7.18 & 0.03 & 7.04 & 0.02 & 7.01 & 0.03 & 6.81 & 0.02 & 6.79 & 0.02 \\
\hline $65 \mathrm{~L} 3$ & 7.69 & 0.04 & 6.98 & 0.02 & 7.46 & 0.02 & 6.81 & 0.02 & 7.20 & 0.02 & 6.65 & 0.01 \\
\hline 66L1 & 7.33 & 0.04 & 7.19 & 0.04 & 7.12 & 0.03 & 7.01 & 0.04 & 6.87 & 0.04 & 6.82 & 0.03 \\
\hline 66L3 & 7.63 & 0.05 & 6.96 & 0.03 & 7.50 & 0.04 & 6.79 & 0.02 & 7.22 & 0.03 & 6.63 & 0.02 \\
\hline
\end{tabular}

SE, standard error;

${ }^{a}$ indicates that the quality of the data is not sufficient to allow an accurate determination

${ }^{b}$, these i-motifs are not stable enough to determine $\mathrm{pH}_{T}$. 
Table S3 $\mathrm{pH}_{T}$ obtained from UV melting and annealing processes at different temperatures. (Continued_02)

\begin{tabular}{|c|c|c|c|c|c|c|c|c|c|c|c|c|}
\hline \multirow{3}{*}{$\begin{array}{c}\text { Temperature } /{ }^{\circ} \mathrm{C} \\
\text { Oligo }\end{array}$} & \multicolumn{4}{|c|}{35} & \multicolumn{4}{|c|}{40} & \multicolumn{4}{|c|}{45} \\
\hline & \multicolumn{2}{|c|}{ heating } & \multicolumn{2}{|c|}{ cooling } & \multicolumn{2}{|c|}{ heating } & \multicolumn{2}{|c|}{ cooling } & \multicolumn{2}{|c|}{ heating } & \multicolumn{2}{|c|}{ cooling } \\
\hline & $\mathrm{pH}_{\mathrm{T}}$ & SE & $\mathrm{pH}_{\mathrm{T}}$ & SE & $\mathrm{pH}_{\mathrm{T}}$ & SE & $\mathrm{pH}_{\mathrm{T}}$ & SE & $\mathrm{pH}_{\mathrm{T}}$ & SE & $\mathrm{pH}_{\mathrm{T}}$ & SE \\
\hline 33L1 & 6.11 & 0.03 & 5.74 & 0.03 & 5.86 & 0.03 & 5.58 & 0.03 & 5.56 & 0.03 & 5.46 & 0.03 \\
\hline 33L3 & 6.07 & 0.04 & 6.08 & 0.04 & 5.81 & 0.03 & 5.84 & 0.04 & 5.59 & 0.00 & 5.62 & 0.03 \\
\hline 34L1 & 6.14 & 0.07 & 5.88 & 0.05 & 5.74 & 0.05 & 5.67 & 0.04 & 5.59 & 0.04 & 5.53 & 0.03 \\
\hline $34 \mathrm{~L} 3$ & 6.17 & 0.01 & 6.17 & 0.01 & 5.93 & 0.02 & 5.94 & 0.02 & 5.66 & 0.02 & 5.72 & 0.02 \\
\hline 43L1 & 6.09 & 0.02 & 6.09 & 0.03 & 5.85 & 0.02 & 5.87 & 0.03 & 5.65 & 0.02 & 5.69 & 0.02 \\
\hline 43L3 & 6.32 & 0.02 & 6.33 & 0.02 & 6.08 & 0.01 & 6.11 & 0.01 & 5.88 & 0.02 & 5.88 & 0.02 \\
\hline 44L1 & 6.19 & 0.03 & 6.17 & 0.01 & 5.99 & 0.02 & 5.98 & 0.01 & 5.82 & 0.02 & 5.79 & 0.01 \\
\hline $44 \mathrm{~L} 3$ & 6.40 & 0.01 & 6.40 & 0.01 & 6.17 & 0.01 & 6.21 & 0.01 & 5.97 & 0.01 & 6.01 & 0.01 \\
\hline $45 \mathrm{~L} 1$ & 6.30 & 0.01 & 6.32 & 0.01 & 6.10 & 0.01 & 6.14 & 0.01 & 5.90 & 0.01 & 5.93 & 0.02 \\
\hline $45 \mathrm{~L} 3$ & 6.45 & 0.02 & 6.44 & 0.02 & 6.25 & 0.02 & 6.27 & 0.02 & 6.05 & 0.01 & 6.07 & 0.01 \\
\hline 54L1 & 6.38 & 0.01 & 6.42 & 0.01 & 6.19 & 0.01 & 6.26 & 0.01 & 6.01 & 0.01 & 6.05 & 0.01 \\
\hline 54L3 & 6.59 & 0.02 & 6.52 & 0.02 & 6.35 & 0.01 & 6.32 & 0.01 & 6.16 & 0.01 & 6.14 & 0.01 \\
\hline 55L1 & 6.43 & 0.01 & 6.48 & 0.01 & 6.28 & 0.01 & 6.30 & 0.01 & 6.11 & 0.01 & 6.11 & 0.01 \\
\hline $55 \mathrm{~L} 3$ & 6.67 & 0.02 & 6.51 & 0.02 & 6.43 & 0.03 & 6.36 & 0.02 & 6.20 & 0.02 & 6.16 & 0.02 \\
\hline $56 \mathrm{~L} 1$ & 6.52 & 0.02 & 6.55 & 0.01 & 6.32 & 0.01 & 6.35 & 0.01 & 6.14 & 0.01 & 6.18 & 0.01 \\
\hline $56 \mathrm{~L} 3$ & 6.74 & 0.01 & 6.51 & 0.01 & 6.50 & 0.01 & 6.35 & 0.01 & 6.25 & 0.01 & 6.18 & 0.01 \\
\hline 65L1 & 6.61 & 0.02 & 6.61 & 0.02 & 6.42 & 0.02 & 6.41 & 0.02 & 6.20 & 0.02 & 6.24 & 0.02 \\
\hline $65 \mathrm{~L} 3$ & 6.91 & 0.02 & 6.51 & 0.01 & 6.62 & 0.01 & 6.38 & 0.01 & 6.37 & 0.01 & 6.19 & 0.01 \\
\hline 66L1 & 6.64 & 0.03 & 6.63 & 0.03 & 6.45 & 0.02 & 6.45 & 0.03 & 6.25 & 0.03 & 6.24 & 0.03 \\
\hline $66 \mathrm{~L} 3$ & 6.96 & 0.03 & 6.49 & 0.02 & 6.67 & 0.02 & 6.35 & 0.02 & 6.42 & 0.02 & 6.18 & 0.02 \\
\hline
\end{tabular}

SE, standard error;

${ }^{a}$ indicates that the quality of the data is not sufficient to allow an accurate determination

${ }^{b}$, these i-motifs are not stable enough to determine $p H_{T}$. 
Table S3 $\mathrm{pH}_{T}$ obtained from UV melting and annealing processes at different temperatures. (Continued_03)

\begin{tabular}{|c|c|c|c|c|c|c|c|c|c|c|c|c|}
\hline \multirow{3}{*}{$\begin{array}{c}\text { Temperature } /{ }^{\circ} \mathrm{C} \\
\text { Oligo }\end{array}$} & \multicolumn{4}{|c|}{50} & \multicolumn{4}{|c|}{55} & \multicolumn{4}{|c|}{60} \\
\hline & \multicolumn{2}{|c|}{ heating } & \multicolumn{2}{|c|}{ cooling } & \multicolumn{2}{|c|}{ heating } & \multicolumn{2}{|c|}{ cooling } & \multicolumn{2}{|c|}{ heating } & \multicolumn{2}{|c|}{ cooling } \\
\hline & $\mathrm{pH}_{\mathrm{T}}$ & SE & $\mathrm{pH}$ & SE & $\mathrm{pH}_{\mathrm{T}}$ & SE & $\mathrm{pH}_{\mathrm{T}}$ & SE & $\mathrm{pH}_{\mathrm{T}}$ & SE & $\mathrm{pH}$ & SE \\
\hline 33L1 & b & $b$ & $b$ & $\mathrm{~b}$ & $\mathrm{~b}$ & $\mathrm{~b}$ & $\mathrm{~b}$ & $b$ & b & $b$ & $\mathrm{~b}$ & b \\
\hline 33L3 & 5.43 & 0.04 & 5.50 & 0.03 & $b$ & $b$ & $b$ & $b$ & b & $b$ & b & b \\
\hline 34L1 & 5.40 & 0.05 & 5.45 & 0.05 & $b$ & $b$ & $b$ & $b$ & $b$ & $b$ & b & $b$ \\
\hline 34L3 & 5.48 & 0.02 & 5.53 & 0.01 & 5.23 & 0.07 & 5.37 & 0.03 & b & b & $b$ & b \\
\hline 43L1 & 5.49 & 0.02 & 5.53 & 0.02 & 5.33 & 0.03 & 5.42 & 0.03 & $b$ & $b$ & $b$ & $b$ \\
\hline 43L3 & 5.62 & 0.01 & 5.67 & 0.01 & 5.41 & 0.02 & 5.51 & 0.00 & b & $b$ & b & b \\
\hline 44L1 & 5.61 & 0.03 & 5.59 & 0.01 & 5.41 & 0.05 & 5.44 & 0.02 & b & b & $b$ & b \\
\hline 44L3 & 5.74 & 0.01 & 5.75 & 0.01 & 5.53 & 0.01 & 5.54 & 0.01 & 5.36 & 0.01 & 5.38 & 0.01 \\
\hline 45L1 & 5.70 & 0.01 & 5.73 & 0.01 & 5.52 & 0.01 & 5.54 & 0.01 & 5.36 & 0.01 & 5.38 & 0.02 \\
\hline $45 \mathrm{~L} 3$ & 5.77 & 0.01 & 5.81 & 0.02 & 5.60 & 0.01 & 5.62 & 0.02 & 5.36 & 0.02 & 5.41 & 0.02 \\
\hline 54L1 & 5.80 & 0.01 & 5.82 & 0.02 & 5.58 & 0.02 & 5.61 & 0.02 & 5.40 & 0.02 & 5.42 & 0.02 \\
\hline 54L3 & 5.93 & 0.01 & 5.91 & 0.02 & 5.69 & 0.01 & 5.69 & 0.01 & 5.45 & 0.02 & 5.46 & 0.02 \\
\hline $55 \mathrm{~L} 1$ & 5.86 & 0.01 & 5.89 & 0.01 & 5.67 & 0.01 & 5.69 & 0.01 & 5.47 & 0.01 & 5.51 & 0.01 \\
\hline 55L3 & 5.95 & 0.03 & 5.96 & 0.02 & 5.73 & 0.02 & 5.73 & 0.02 & 5.57 & 0.01 & 5.59 & 0.00 \\
\hline $56 \mathrm{~L} 1$ & 5.96 & 0.02 & 5.96 & 0.01 & 5.74 & 0.01 & 5.76 & 0.01 & 5.57 & 0.01 & 5.60 & 0.01 \\
\hline $56 \mathrm{~L} 3$ & 6.03 & 0.01 & 5.99 & 0.01 & 5.77 & 0.01 & 5.76 & 0.01 & 5.57 & 0.01 & 5.58 & 0.01 \\
\hline 65L1 & 6.00 & 0.00 & 6.11 & 0.00 & 5.86 & 0.02 & 5.87 & 0.02 & 5.62 & 0.01 & 5.64 & 0.01 \\
\hline $65 \mathrm{~L} 3$ & 6.11 & 0.01 & 6.05 & 0.01 & 5.86 & 0.01 & 5.81 & 0.01 & 5.65 & 0.01 & 5.65 & 0.01 \\
\hline 66L1 & 6.06 & 0.02 & 6.09 & 0.02 & 5.85 & 0.02 & 5.87 & 0.02 & 5.72 & 0.01 & 5.74 & 0.00 \\
\hline 66L3 & 6.20 & 0.02 & 6.07 & 0.02 & 5.91 & 0.03 & 5.77 & 0.02 & 5.66 & 0.01 & 5.64 & 0.01 \\
\hline
\end{tabular}

SE, standard error;

${ }^{a}$ indicates that the quality of the data is not sufficient to allow an accurate determination

${ }^{b}$, these i-motifs are not stable enough to determine $p H_{T}$. 
Table S3 $\mathrm{pH}_{T}$ obtained from UV melting and annealing processes at different temperatures. (Continued_04)

\begin{tabular}{|c|c|c|c|c|c|c|c|c|}
\hline \multirow{3}{*}{$\begin{array}{c}\text { Temperature } /{ }^{\circ} \mathrm{C} \\
\text { Oligo }\end{array}$} & \multicolumn{4}{|c|}{65} & \multicolumn{4}{|c|}{70} \\
\hline & \multicolumn{2}{|c|}{ heating } & \multicolumn{2}{|c|}{ cooling } & \multicolumn{2}{|c|}{ heating } & \multicolumn{2}{|c|}{ cooling } \\
\hline & $\mathrm{pH}_{\mathrm{T}}$ & SE & $\mathrm{pH}_{\mathrm{T}}$ & SE & $\mathrm{pH}_{\mathrm{T}}$ & SE & $\mathrm{pH}_{\mathrm{T}}$ & SE \\
\hline $33 \mathrm{~L} 1$ & $b$ & $b$ & $b$ & $b$ & $b$ & $b$ & $b$ & $b$ \\
\hline 33L3 & $b$ & $b$ & $b$ & $b$ & $b$ & $b$ & $b$ & $b$ \\
\hline 34L1 & $b$ & $b$ & $b$ & $b$ & $b$ & $b$ & $b$ & $b$ \\
\hline $34 \mathrm{~L} 3$ & $b$ & $b$ & $b$ & $b$ & $b$ & $b$ & $b$ & $b$ \\
\hline 43L1 & $b$ & $b$ & $b$ & $b$ & $b$ & $b$ & $b$ & $b$ \\
\hline 43L3 & $b$ & $b$ & $b$ & $b$ & b & $b$ & $b$ & $b$ \\
\hline 44L1 & $b$ & $b$ & $b$ & $b$ & $b$ & $b$ & $b$ & $b$ \\
\hline 44L3 & $b$ & $b$ & $b$ & $b$ & $b$ & $b$ & $b$ & $b$ \\
\hline 45L1 & $b$ & $b$ & $b$ & $b$ & $b$ & $b$ & $b$ & $b$ \\
\hline $45 \mathrm{~L} 3$ & 5.33 & 0.00 & 5.30 & 0.03 & b & $b$ & $b$ & $b$ \\
\hline $54 \mathrm{~L} 1$ & 5.28 & 0.02 & 5.33 & 0.00 & b & $b$ & $b$ & $b$ \\
\hline $54 \mathrm{~L} 3$ & 5.24 & 0.02 & 5.33 & 0.17 & $b$ & $b$ & $b$ & $b$ \\
\hline $55 \mathrm{~L} 1$ & 5.24 & 0.02 & 5.25 & 0.02 & $b$ & $\mathrm{~b}$ & $b$ & $b$ \\
\hline $55 \mathrm{~L} 3$ & 5.39 & 0.02 & 5.39 & 0.02 & b & $b$ & $b$ & $b$ \\
\hline $56 \mathrm{~L} 1$ & 5.39 & 0.02 & 5.40 & 0.01 & $b$ & $b$ & $b$ & $b$ \\
\hline $56 \mathrm{~L} 3$ & 5.37 & 0.01 & 5.39 & 0.01 & $b$ & $b$ & $b$ & $\mathrm{~b}$ \\
\hline $65 \mathrm{~L} 1$ & 5.48 & 0.01 & 5.49 & 0.01 & 5.36 & 0.01 & 5.30 & 0.01 \\
\hline $65 \mathrm{~L} 3$ & 5.48 & 0.03 & 5.47 & 0.01 & 5.33 & 0.01 & 5.33 & 0.00 \\
\hline $66 \mathrm{~L} 1$ & 5.50 & 0.01 & 5.49 & 0.01 & 5.44 & 0.00 & 5.34 & 0.00 \\
\hline 66L3 & 5.45 & 0.01 & 5.43 & 0.01 & 5.23 & 0.01 & 5.28 & 0.01 \\
\hline
\end{tabular}

SE, standard error;

${ }^{a}$ indicates that the quality of the data is not sufficient to allow an accurate determination

${ }^{b}$, these i-motifs are not stable enough to determine $\mathrm{pH}_{T}$. 
Table S4 Linear fitting results of standard $\Delta G^{\circ}$ as a function of $\mathrm{pH} .{ }^{a}$

\begin{tabular}{ccccccc}
\hline \multirow{2}{*}{ Name } & \multicolumn{3}{c}{$\Delta \mathrm{G}^{\circ}\left(20^{\circ} \mathrm{C}\right)$} & & \multicolumn{1}{c}{$\Delta \mathrm{G}^{\circ}\left(37^{\circ} \mathrm{C}\right)$} \\
\cline { 2 - 7 } & Slope & Intercept & Pearson's r & Slope & Intercept & Pearson's r \\
\hline 33L3 & $25.50 \pm 0.82$ & $-169.76 \pm 5.17$ & 0.99173 & $29.95 \pm 0.89$ & $-175.84 \pm 5.56$ & 0.99306 \\
34L3 & $28.96 \pm 0.96$ & $-195.95 \pm 6.06$ & 0.99081 & $33.18 \pm 0.74$ & $-198.32 \pm 4.68$ & 0.99580 \\
43L3 & $26.58 \pm 1.11$ & $-186.51 \pm 7.11$ & 0.98394 & $29.44 \pm 0.69$ & $-180.74 \pm 4.47$ & 0.99478 \\
44L3 & $29.46 \pm 1.41$ & $-208.45 \pm 9.04$ & 0.97889 & $32.63 \pm 0.89$ & $-202.90 \pm 5.68$ & 0.99305 \\
\hline
\end{tabular}

${ }^{a}$ Linear fitting results obtained from Figures 9 A and 9B in the main manuscript, respectively. 
Table S5.1 $T_{1 / 2}\left({ }^{\circ} \mathrm{C}\right)$ of DAP obtained from UV melting and annealing processes at different pHs.

\begin{tabular}{|c|c|c|c|c|c|c|}
\hline $\mathrm{pH}$ & $\mathrm{T}_{1 / 2}$, heating & Standard Error & $\mathrm{T}_{1 / 2}$, cooling & Standard Error & $T_{m}$ & $T_{\text {hysteresis }}$ \\
\hline 5.01 & 75.52 & 0.44 & 75.71 & 1.64 & 75.62 & -0.19 \\
\hline 5.15 & 70.78 & 0.37 & 70.45 & 0.70 & 70.62 & 0.32 \\
\hline 5.34 & 66.35 & 0.25 & 65.48 & 0.42 & 65.92 & 0.87 \\
\hline 5.51 & 62.04 & 0.22 & 61.21 & 0.27 & 61.62 & 0.83 \\
\hline 5.59 & 60.57 & 0.22 & 59.66 & 0.26 & 60.11 & 0.91 \\
\hline 5.71 & 56.55 & 0.22 & 56.13 & 0.20 & 56.34 & 0.42 \\
\hline 5.79 & 55.09 & 0.18 & 54.47 & 0.18 & 54.78 & 0.61 \\
\hline 5.90 & 52.19 & 0.22 & 52.26 & 0.18 & 52.23 & -0.08 \\
\hline 6.00 & 50.11 & 0.21 & 50.13 & 0.18 & 50.12 & -0.02 \\
\hline 6.11 & 48.03 & 0.20 & 48.16 & 0.16 & 48.09 & -0.13 \\
\hline 6.19 & 45.96 & 0.20 & 46.15 & 0.16 & 46.05 & -0.18 \\
\hline 6.30 & 43.15 & 0.13 & 43.62 & 0.14 & 43.39 & -0.47 \\
\hline 6.40 & 41.06 & 0.18 & 41.16 & 0.15 & 41.11 & -0.11 \\
\hline 6.51 & 38.30 & 0.16 & 38.31 & 0.14 & 38.31 & -0.01 \\
\hline 6.61 & 35.75 & 0.12 & 35.81 & 0.15 & 35.78 & -0.06 \\
\hline 6.70 & 33.28 & 0.11 & 33.25 & 0.16 & 33.27 & 0.03 \\
\hline 6.80 & 31.34 & 0.07 & 31.16 & 0.11 & 31.25 & 0.18 \\
\hline 6.90 & 28.80 & 0.11 & 28.85 & 0.14 & 28.83 & -0.06 \\
\hline 7.01 & 26.44 & 0.10 & 26.55 & 0.12 & 26.50 & -0.11 \\
\hline 7.10 & 24.67 & 0.09 & 24.24 & 0.13 & 24.45 & 0.43 \\
\hline 7.21 & 22.30 & 0.08 & 21.65 & 0.10 & 21.98 & 0.65 \\
\hline 7.30 & 20.50 & 0.05 & 19.58 & 0.09 & 20.04 & 0.92 \\
\hline 7.40 & 18.22 & 0.06 & 17.02 & 0.08 & 17.62 & 1.20 \\
\hline 7.50 & 16.58 & 0.06 & 14.83 & 0.05 & 15.71 & 1.75 \\
\hline 7.72 & 13.27 & 0.05 & 11.45 & 0.16 & 12.36 & 1.82 \\
\hline 7.98 & -- & -- & -- & -- & -- & -- \\
\hline
\end{tabular}

$T_{m}=\left(T_{1 / 2, \text { heating }}+T_{1 / 2, \text { cooling }}\right) / 2$;

$\mathrm{T}_{\text {hysteresis }}=T_{1 / 2 \text {, heating }}-T_{1 / 2 \text {, cooling. }}$ 
Table S5.2 $T_{1 / 2}\left({ }^{\circ} \mathrm{C}\right)$ of PDGF-A obtained from UV melting and annealing processes at different pHs.

\begin{tabular}{|c|c|c|c|c|c|c|}
\hline $\mathrm{pH}$ & $\mathrm{T}_{1 / 2}$, heating & Standard Error & $\mathrm{T}_{1 / 2}$, cooling & Standard Error & $T_{m}$ & $T_{\text {hysteresis }}$ \\
\hline 5.01 & 78.36 & 0.58 & 77.99 & 2.01 & 78.17 & 0.37 \\
\hline 5.15 & 72.36 & 0.65 & 71.27 & 0.92 & 71.81 & 1.09 \\
\hline 5.34 & 66.04 & 0.42 & 65.47 & 0.51 & 65.75 & 0.57 \\
\hline 5.51 & 61.39 & 0.38 & 61.18 & 0.43 & 61.29 & 0.21 \\
\hline 5.59 & 59.80 & 0.42 & 59.56 & 0.47 & 59.68 & 0.23 \\
\hline 5.71 & 56.47 & 0.34 & 56.31 & 0.41 & 56.39 & 0.16 \\
\hline 5.79 & 54.53 & 0.31 & 54.37 & 0.38 & 54.45 & 0.16 \\
\hline 5.90 & 52.26 & 0.35 & 52.38 & 0.38 & 52.32 & -0.12 \\
\hline 6.00 & 49.99 & 0.30 & 50.23 & 0.30 & 50.11 & -0.23 \\
\hline 6.11 & 47.77 & 0.39 & 47.55 & 0.30 & 47.66 & 0.22 \\
\hline 6.19 & 45.20 & 0.27 & 45.82 & 0.26 & 45.51 & -0.62 \\
\hline 6.30 & 42.84 & 0.27 & 43.33 & 0.24 & 43.08 & -0.50 \\
\hline 6.40 & 40.41 & 0.22 & 40.71 & 0.25 & 40.56 & -0.30 \\
\hline 6.51 & 37.70 & 0.18 & 37.74 & 0.21 & 37.72 & -0.04 \\
\hline 6.61 & 35.26 & 0.19 & 35.08 & 0.19 & 35.17 & 0.18 \\
\hline 6.70 & 33.43 & 0.08 & 32.57 & 0.17 & 33.00 & 0.86 \\
\hline 6.80 & 31.39 & 0.10 & 30.27 & 0.16 & 30.83 & 1.12 \\
\hline 6.90 & 28.92 & 0.12 & 27.68 & 0.13 & 28.30 & 1.24 \\
\hline 7.01 & 26.68 & 0.13 & 24.89 & 0.12 & 25.78 & 1.79 \\
\hline 7.10 & 24.87 & 0.12 & 22.38 & 0.11 & 23.62 & 2.49 \\
\hline 7.21 & 22.65 & 0.09 & 19.48 & 0.09 & 21.06 & 3.17 \\
\hline 7.30 & 20.50 & 0.13 & 17.14 & 0.07 & 18.82 & 3.35 \\
\hline 7.40 & 18.27 & 0.13 & 14.15 & 0.07 & 16.21 & 4.11 \\
\hline 7.50 & 16.11 & 0.11 & 11.37 & 0.07 & 13.74 & 4.74 \\
\hline 7.72 & 12.50 & 0.08 & 7.61 & 0.12 & 10.05 & 4.88 \\
\hline 7.98 & - & - & - & - & -- & -- \\
\hline
\end{tabular}

$T_{m}=\left(T_{1 / 2, \text { heating }}+T_{1 / 2, \text { cooling }}\right) / 2$;

$T_{\text {hysteresis }}=T_{1 / 2, \text { heating }}-T_{1 / 2, \text { cooling. }}$ 
Table S5.3 $T_{1 / 2}\left({ }^{\circ} \mathrm{C}\right)$ of KRAS obtained from UV melting and annealing processes at different pHs.

\begin{tabular}{|c|c|c|c|c|c|c|}
\hline $\mathrm{pH}$ & $\mathrm{T}_{1 / 2}$, heating & Standard Error & $\mathrm{T}_{1 / 2}$, cooling & Standard Error & $T_{m}$ & $T_{\text {hysteresis }}$ \\
\hline 5.01 & 64.05 & 0.74 & 61.38 & 0.42 & 62.71 & 2.66 \\
\hline 5.15 & 60.78 & 0.67 & 58.26 & 0.41 & 59.52 & 2.52 \\
\hline 5.34 & 57.43 & 0.76 & 54.74 & 0.50 & 56.08 & 2.69 \\
\hline 5.51 & 54.18 & 0.57 & 51.47 & 0.48 & 52.83 & 2.71 \\
\hline 5.59 & 52.69 & 0.55 & 50.04 & 0.46 & 51.36 & 2.64 \\
\hline 5.71 & 50.08 & 0.43 & 47.33 & 0.41 & 48.71 & 2.75 \\
\hline 5.79 & 48.73 & 0.43 & 45.94 & 0.41 & 47.34 & 2.79 \\
\hline 5.90 & 46.98 & 0.37 & 44.17 & 0.39 & 45.57 & 2.81 \\
\hline 6.00 & 45.82 & 0.39 & 43.30 & 0.30 & 44.56 & 2.52 \\
\hline 6.11 & 43.31 & 0.35 & 40.85 & 0.27 & 42.08 & 2.46 \\
\hline 6.19 & 41.84 & 0.36 & 38.88 & 0.25 & 40.36 & 2.96 \\
\hline 6.30 & 40.30 & 0.35 & 36.40 & 0.22 & 38.35 & 3.90 \\
\hline 6.40 & 38.47 & 0.34 & 33.66 & 0.21 & 36.06 & 4.81 \\
\hline 6.51 & 36.17 & 0.29 & 30.48 & 0.17 & 33.33 & 5.68 \\
\hline 6.61 & 34.13 & 0.29 & 27.53 & 0.14 & 30.83 & 6.60 \\
\hline 6.70 & 32.48 & 0.25 & 25.07 & 0.13 & 28.77 & 7.41 \\
\hline 6.80 & 30.66 & 0.26 & 22.49 & 0.14 & 26.57 & 8.17 \\
\hline 6.90 & 28.45 & 0.27 & 19.99 & 0.12 & 24.22 & 8.46 \\
\hline 7.01 & 26.45 & 0.26 & 17.24 & 0.10 & 21.84 & 9.21 \\
\hline 7.10 & 24.99 & 0.21 & 14.84 & 0.10 & 19.92 & 10.15 \\
\hline 7.21 & 22.92 & 0.22 & 12.10 & 0.13 & 17.51 & 10.82 \\
\hline 7.30 & 21.23 & 0.25 & 10.17 & 0.16 & 15.70 & 11.06 \\
\hline 7.40 & 19.14 & 0.27 & 7.98 & 0.36 & 13.56 & 11.16 \\
\hline 7.50 & -- & -- & -- & -- & -- & -- \\
\hline 7.72 & -- & -- & -- & -- & -- & -- \\
\hline 7.98 & -- & -- & -- & -- & -- & -- \\
\hline
\end{tabular}

$T_{m}=\left(T_{1 / 2, \text { heating }}+T_{1 / 2, \text { cooling }}\right) / 2$;

$\mathrm{T}_{\text {hysteresis }}=T_{1 / 2, \text { heating }}-T_{1 / 2}$, cooling. 
Table S5.4 $T_{1 / 2}\left({ }^{\circ} \mathrm{C}\right)$ of HIF-1 $\alpha$ obtained from UV melting and annealing processes at different pHs.

\begin{tabular}{|c|c|c|c|c|c|c|}
\hline $\mathrm{pH}$ & $\mathrm{T}_{1 / 2}$, heating & Standard Error & $\mathrm{T}_{1 / 2}$, cooling & Standard Error & $T_{m}$ & $T_{\text {hysteresis }}$ \\
\hline 5.01 & 62.57 & 0.55 & 62.37 & 0.48 & 62.47 & 0.19 \\
\hline 5.15 & 60.24 & 0.44 & 59.33 & 0.42 & 59.79 & 0.91 \\
\hline 5.34 & 56.64 & 0.34 & 56.06 & 0.35 & 56.35 & 0.58 \\
\hline 5.51 & 52.66 & 0.31 & 52.52 & 0.30 & 52.59 & 0.15 \\
\hline 5.59 & 51.56 & 0.29 & 51.40 & 0.30 & 51.48 & 0.17 \\
\hline 5.71 & 48.68 & 0.25 & 48.60 & 0.27 & 48.64 & 0.08 \\
\hline 5.79 & 47.04 & 0.22 & 47.11 & 0.26 & 47.08 & -0.07 \\
\hline 5.90 & 45.10 & 0.19 & 45.26 & 0.25 & 45.18 & -0.15 \\
\hline 6.00 & 43.13 & 0.27 & 44.15 & 0.22 & 43.64 & -1.03 \\
\hline 6.11 & 40.26 & 0.25 & 41.29 & 0.20 & 40.77 & -1.04 \\
\hline 6.19 & 38.84 & 0.20 & 39.67 & 0.21 & 39.26 & -0.83 \\
\hline 6.30 & 36.86 & 0.18 & 37.72 & 0.18 & 37.29 & -0.87 \\
\hline 6.40 & 34.74 & 0.17 & 35.51 & 0.17 & 35.13 & -0.77 \\
\hline 6.51 & 32.56 & 0.08 & 32.90 & 0.16 & 32.73 & -0.34 \\
\hline 6.61 & 30.91 & 0.07 & 30.81 & 0.15 & 30.86 & 0.10 \\
\hline 6.70 & 29.19 & 0.10 & 28.66 & 0.13 & 28.92 & 0.54 \\
\hline 6.80 & 27.32 & 0.09 & 26.79 & 0.13 & 27.05 & 0.53 \\
\hline 6.90 & 25.20 & 0.10 & 24.35 & 0.15 & 24.78 & 0.85 \\
\hline 7.01 & 22.98 & 0.08 & 22.29 & 0.12 & 22.63 & 0.69 \\
\hline 7.10 & 21.61 & 0.08 & 20.23 & 0.13 & 20.92 & 1.37 \\
\hline 7.21 & 19.43 & 0.08 & 17.70 & 0.10 & 18.57 & 1.73 \\
\hline 7.30 & 17.97 & 0.11 & 16.03 & 0.11 & 17.00 & 1.94 \\
\hline 7.40 & 15.86 & 0.12 & 13.84 & 0.09 & 14.85 & 2.03 \\
\hline 7.50 & 14.12 & 0.09 & 11.85 & 0.11 & 12.99 & 2.27 \\
\hline 7.72 & 11.67 & 0.16 & 9.05 & 0.12 & 10.36 & 2.61 \\
\hline 7.98 & -- & -- & -- & -- & -- & -- \\
\hline
\end{tabular}

$T_{m}=\left(T_{1 / 2, \text { heating }}+T_{1 / 2, \text { cooling }}\right) / 2$;

$\mathrm{T}_{\text {hysteresis }}=T_{1 / 2, \text { heating }}-T_{1 / 2}$, cooling. 
Table S5.5 $T_{1 / 2}\left({ }^{\circ} \mathrm{C}\right)$ of $B C L 2$ obtained from UV melting and annealing processes at different pHs.

\begin{tabular}{|c|c|c|c|c|c|c|}
\hline $\mathrm{pH}$ & $\mathbf{T}_{1 / 2}$, heating & Standard Error & $\mathrm{T}_{1 / 2}$, cooling & Standard Error & $\mathrm{T}_{\mathrm{m}}$ & $T_{\text {hysteresis }}$ \\
\hline 5.01 & 61.56 & 0.28 & 60.43 & 0.31 & 61.00 & 1.13 \\
\hline 5.15 & 58.33 & 0.36 & 58.32 & 0.30 & 58.33 & 0.01 \\
\hline 5.34 & 53.84 & 0.30 & 54.07 & 0.23 & 53.95 & -0.23 \\
\hline 5.51 & 49.70 & 0.24 & 49.66 & 0.20 & 49.68 & 0.04 \\
\hline 5.59 & 47.99 & 0.20 & 48.05 & 0.17 & 48.02 & -0.06 \\
\hline 5.71 & 44.82 & 0.19 & 44.90 & 0.17 & 44.86 & -0.08 \\
\hline 5.79 & 42.95 & 0.13 & 43.03 & 0.13 & 42.99 & -0.08 \\
\hline 5.90 & 40.69 & 0.10 & 40.43 & 0.14 & 40.56 & 0.26 \\
\hline 6.00 & 38.75 & 0.08 & 38.21 & 0.15 & 38.48 & 0.54 \\
\hline 6.11 & 36.00 & 0.07 & 34.85 & 0.13 & 35.42 & 1.15 \\
\hline 6.19 & 33.22 & 0.09 & 33.01 & 0.12 & 33.12 & 0.21 \\
\hline 6.30 & 31.20 & 0.09 & 30.45 & 0.10 & 30.83 & 0.75 \\
\hline 6.40 & 29.20 & 0.08 & 27.77 & 0.10 & 28.49 & 1.43 \\
\hline 6.51 & 26.29 & 0.08 & 24.74 & 0.10 & 25.51 & 1.55 \\
\hline 6.61 & 23.70 & 0.07 & 22.04 & 0.08 & 22.87 & 1.66 \\
\hline 6.70 & 21.81 & 0.12 & 19.68 & 0.07 & 20.75 & 2.13 \\
\hline 6.80 & 18.56 & 0.16 & 17.31 & 0.06 & 17.93 & 1.24 \\
\hline 6.90 & 17.25 & 0.10 & 14.51 & 0.10 & 15.88 & 2.73 \\
\hline 7.01 & 15.09 & 0.08 & 11.95 & 0.14 & 13.52 & 3.14 \\
\hline 7.10 & 13.03 & 0.21 & 9.73 & 0.11 & 11.38 & 3.30 \\
\hline 7.21 & 10.73 & 0.31 & -- & -- & -- & -- \\
\hline 7.30 & -- & -- & -- & -- & -- & -- \\
\hline 7.40 & -- & -- & -- & -- & -- & -- \\
\hline 7.50 & -- & -- & -- & -- & -- & -- \\
\hline 7.72 & -- & -- & -- & -- & -- & -- \\
\hline 7.98 & -- & -- & -- & -- & -- & -- \\
\hline
\end{tabular}

$T_{m}=\left(T_{1 / 2, \text { heating }}+T_{1 / 2, \text { cooling }}\right) / 2$;

$\mathrm{T}_{\text {hysteresis }}=T_{1 / 2, \text { heating }}-T_{1 / 2}$, cooling. 
Table S6.1 $\mathrm{pH}_{T}$ of DAP obtained from UV melting and annealing processes at different temperatures.

\begin{tabular}{|c|c|c|c|c|c|c|}
\hline $\begin{array}{c}\text { Temperature, } \\
{ }^{\circ} \mathrm{C}\end{array}$ & $\begin{array}{c}\mathrm{pH} \text {, } \\
\text { heating }\end{array}$ & $\begin{array}{c}\text { Standard } \\
\text { Error }\end{array}$ & $\begin{array}{c}\mathrm{pH}_{\mathrm{T}} \\
\text { cooling }\end{array}$ & $\begin{array}{l}\text { Standard } \\
\text { Error }\end{array}$ & $\begin{array}{c}\text { Averaged } \\
\mathrm{pH}_{\mathrm{T}}\end{array}$ & pH hysteresis \\
\hline 10 & -- & -- & -- & -- & -- & -- \\
\hline 15 & -- & -- & 7.61 & 0.17 & -- & -- \\
\hline 20 & 7.27 & 0.03 & 7.23 & 0.04 & 7.25 & 0.04 \\
\hline 25 & 7.05 & 0.02 & 7.05 & 0.03 & 7.05 & 0.00 \\
\hline 30 & 6.83 & 0.02 & 6.81 & 0.02 & 6.82 & 0.01 \\
\hline 35 & 6.64 & 0.02 & 6.65 & 0.02 & 6.64 & -0.01 \\
\hline 40 & 6.47 & 0.02 & 6.43 & 0.02 & 6.45 & 0.04 \\
\hline 45 & 6.23 & 0.02 & 6.25 & 0.02 & 6.24 & -0.02 \\
\hline 50 & 6.02 & 0.01 & 6.02 & 0.01 & 6.02 & -0.01 \\
\hline 55 & 5.80 & 0.01 & 5.80 & 0.01 & 5.80 & 0.00 \\
\hline 60 & 5.64 & 0.01 & 5.63 & 0.01 & 5.64 & 0.01 \\
\hline 65 & 5.46 & 0.01 & 5.43 & 0.02 & 5.45 & 0.03 \\
\hline 70 & -- & -- & -- & -- & -- & -- \\
\hline
\end{tabular}

Averaged $p H_{T}=\left(p H_{T, \text { heating }}+p H_{T, \text { cooling }}\right) / 2$

$\mathrm{pH}_{\text {hysteresis }}=p H_{T, \text { heating }}-p H_{T, \text { cooling }}$. 
Table S6.2 $\mathrm{pH}_{T}$ of PDGF-A obtained from UV melting and annealing processes at different temperatures.

\begin{tabular}{|c|c|c|c|c|c|c|}
\hline $\begin{array}{c}\text { Temperature, } \\
{ }^{\circ} \mathrm{C}\end{array}$ & $\begin{array}{c}\mathrm{pH}_{\mathrm{T}} \\
\text { heating }\end{array}$ & $\begin{array}{c}\text { Standard } \\
\text { Error }\end{array}$ & $\begin{array}{c}\mathrm{pH}_{\mathrm{T}} \\
\text { cooling }\end{array}$ & $\begin{array}{c}\text { Standard } \\
\text { Error }\end{array}$ & $\begin{array}{c}\text { Averaged } \\
\mathrm{pH}_{\mathrm{T}}\end{array}$ & pH hysteresis \\
\hline 10 & -- & -- & -- & -- & -- & -- \\
\hline 15 & -- & -- & -- & -- & -- & -- \\
\hline 20 & 7.36 & 0.19 & 7.17 & 0.16 & 7.26 & 0.19 \\
\hline 25 & 6.99 & 0.08 & 6.92 & 0.07 & 6.96 & 0.07 \\
\hline 30 & 6.77 & 0.05 & 6.69 & 0.05 & 6.73 & 0.08 \\
\hline 35 & 6.55 & 0.04 & 6.53 & 0.03 & 6.54 & 0.02 \\
\hline 40 & 6.35 & 0.03 & 6.36 & 0.03 & 6.36 & -0.01 \\
\hline 45 & 6.15 & 0.03 & 6.19 & 0.02 & 6.17 & -0.03 \\
\hline 50 & 6.00 & 0.02 & 5.97 & 0.02 & 5.99 & 0.02 \\
\hline 55 & 5.80 & 0.01 & 5.81 & 0.01 & 5.81 & -0.01 \\
\hline 60 & 5.61 & 0.01 & 5.65 & 0.01 & 5.63 & -0.04 \\
\hline 65 & -- & -- & -- & -- & -- & -- \\
\hline 70 & -- & -- & -- & -- & -- & -- \\
\hline
\end{tabular}

Averaged $p H_{T}=\left(p H_{T, \text { heating }}+p H_{T, \text { cooling }}\right) / 2$

$\mathrm{pH}_{\text {hysteresis }}=p H_{T, \text { heating }}-p H_{T, \text { cooling }}$. 
Table S6.3 $\mathrm{pH}_{T}$ of KRAS obtained from UV melting and annealing processes at different temperatures.

\begin{tabular}{|c|c|c|c|c|c|c|}
\hline $\begin{array}{c}\text { Temperature, } \\
{ }^{\circ} \mathrm{C} \\
\end{array}$ & $\begin{array}{c}\mathrm{pH}_{\mathrm{T}}, \\
\text { heating }\end{array}$ & $\begin{array}{c}\text { Standard } \\
\text { Error }\end{array}$ & $\begin{array}{c}\mathrm{pH}_{\mathrm{T}}, \\
\text { cooling }\end{array}$ & $\begin{array}{c}\text { Standard } \\
\text { Error }\end{array}$ & $\begin{array}{c}\text { Averaged } \\
\mathrm{pH}_{\mathrm{T}} \\
\end{array}$ & $\mathrm{pH}_{\text {hysteresis }}$ \\
\hline 10 & -- & -- & -- & -- & -- & -- \\
\hline 15 & -- & -- & 7.01 & 0.16 & -- & -- \\
\hline 20 & 7.32 & 0.15 & 6.68 & 0.09 & 7.00 & 0.63 \\
\hline 25 & 6.86 & 0.13 & 6.51 & 0.07 & 6.68 & 0.35 \\
\hline 30 & 6.59 & 0.10 & 6.33 & 0.05 & 6.46 & 0.26 \\
\hline 35 & 6.39 & 0.07 & 6.19 & 0.04 & 6.29 & 0.19 \\
\hline 40 & 6.18 & 0.04 & 6.00 & 0.04 & 6.09 & 0.18 \\
\hline 45 & 5.96 & 0.04 & 5.84 & 0.04 & 5.90 & 0.12 \\
\hline 50 & 5.78 & 0.03 & 5.69 & 0.03 & 5.74 & 0.09 \\
\hline 55 & 5.58 & 0.01 & 5.49 & 0.02 & 5.53 & 0.09 \\
\hline 60 & -- & -- & -- & -- & -- & -- \\
\hline 65 & -- & -- & -- & -- & -- & -- \\
\hline 70 & -- & -- & -- & -- & -- & -- \\
\hline
\end{tabular}

Averaged $p H_{T}=\left(p H_{T, \text { heating }}+p H_{T, \text { cooling }}\right) / 2$;

$\mathrm{pH}_{\text {hysteresis }}=p H_{T, \text { heating }}-p H_{T, \text { cooling }}$. 
Table S6.4 $\mathrm{pH}_{T}$ of $H I F-1 \alpha$ obtained from UV melting and annealing processes at different temperatures.

\begin{tabular}{|c|c|c|c|c|c|c|}
\hline $\begin{array}{c}\text { Temperature, } \\
{ }^{\circ} \mathrm{C}\end{array}$ & $\begin{array}{c}\mathrm{pH} \text {, } \\
\text { heating }\end{array}$ & $\begin{array}{c}\text { Standard } \\
\text { Error }\end{array}$ & $\begin{array}{c}\mathrm{pH}_{\mathrm{T}} \\
\text { cooling }\end{array}$ & $\begin{array}{l}\text { Standard } \\
\text { Error }\end{array}$ & $\begin{array}{c}\text { Averaged } \\
\mathrm{pH}_{\mathrm{T}}\end{array}$ & pH hysteresis \\
\hline 10 & -- & -- & -- & -- & -- & -- \\
\hline 15 & -- & -- & 7.36 & 0.23 & -- & -- \\
\hline 20 & 7.02 & 0.05 & 7.02 & 0.08 & 7.02 & 0.01 \\
\hline 25 & 6.83 & 0.03 & 6.80 & 0.04 & 6.82 & 0.04 \\
\hline 30 & 6.59 & 0.03 & 6.58 & 0.04 & 6.59 & 0.01 \\
\hline 35 & 6.38 & 0.02 & 6.39 & 0.03 & 6.38 & -0.01 \\
\hline 40 & 6.12 & 0.02 & 6.15 & 0.02 & 6.14 & -0.03 \\
\hline 45 & 5.93 & 0.02 & 5.93 & 0.02 & 5.93 & 0.01 \\
\hline 50 & 5.73 & 0.02 & 5.73 & 0.02 & 5.73 & 0.00 \\
\hline 55 & 5.47 & 0.05 & 5.50 & 0.05 & 5.48 & -0.03 \\
\hline 60 & -- & -- & -- & -- & -- & -- \\
\hline 65 & -- & -- & -- & -- & -- & -- \\
\hline 70 & -- & -- & -- & -- & -- & -- \\
\hline
\end{tabular}

Averaged $p H_{T}=\left(p H_{T, \text { heating }}+p H_{T, \text { cooling }}\right) / 2$

$\mathrm{pH}_{\text {hysteresis }}=p H_{T, \text { heating }}-p H_{T, \text { cooling }}$. 
Table S6.5 $\mathrm{pH}_{T}$ of BCL2 obtained from UV melting and annealing processes at different temperatures.

\begin{tabular}{|c|c|c|c|c|c|c|}
\hline $\begin{array}{c}\text { Temperature, } \\
{ }^{\circ} \mathrm{C}\end{array}$ & $\begin{array}{c}\mathrm{pH}_{\mathrm{T}}, \\
\text { heating }\end{array}$ & $\begin{array}{c}\text { Standard } \\
\text { Error }\end{array}$ & $\begin{array}{c}\mathrm{pH}_{\mathrm{T}} \\
\text { cooling }\end{array}$ & $\begin{array}{c}\text { Standard } \\
\text { Error }\end{array}$ & $\begin{array}{c}\text { Averaged } \\
\mathrm{pH}_{\mathrm{T}}\end{array}$ & pH hysteresis \\
\hline 10 & 7.13 & 0.12 & 6.93 & 0.05 & 7.03 & 0.20 \\
\hline 15 & 6.85 & 0.05 & 6.78 & 0.04 & 6.81 & 0.07 \\
\hline 20 & 6.63 & 0.03 & 6.60 & 0.03 & 6.62 & 0.04 \\
\hline 25 & 6.50 & 0.03 & 6.46 & 0.02 & 6.48 & 0.04 \\
\hline 30 & 6.31 & 0.02 & 6.29 & 0.02 & 6.30 & 0.02 \\
\hline 35 & 6.13 & 0.02 & 6.09 & 0.02 & 6.11 & 0.04 \\
\hline 40 & 5.93 & 0.02 & 5.94 & 0.02 & 5.94 & -0.01 \\
\hline 45 & 5.71 & 0.02 & 5.73 & 0.02 & 5.72 & -0.02 \\
\hline 50 & 5.51 & 0.04 & 5.50 & 0.04 & 5.51 & 0.01 \\
\hline 55 & -- & -- & -- & -- & -- & -- \\
\hline 60 & -- & -- & -- & -- & -- & -- \\
\hline 65 & -- & -- & -- & -- & -- & -- \\
\hline 70 & -- & -- & -- & -- & -- & -- \\
\hline
\end{tabular}

Averaged $p H_{T}=\left(p H_{T, \text { heating }}+p H_{T, \text { cooling }}\right) / 2$

$\mathrm{pH}_{\text {hysteresis }}=p H_{T, \text { heating }}-p H_{T, \text { cooling }}$. 
Table S7 $T_{1 / 2}\left({ }^{\circ} \mathrm{C}\right)$ obtained from UV melting and annealing processes under crowding environments.

\begin{tabular}{|c|c|c|c|c|c|c|c|}
\hline $\begin{array}{c}\text { Crowding } \\
\text { agent }\end{array}$ & Promoter & $\begin{array}{c}\mathrm{T}_{1 / 2}, \\
\text { heating }\end{array}$ & $\begin{array}{c}\text { Standard } \\
\text { Error }\end{array}$ & $\begin{array}{c}\mathrm{T}_{1 / 2}, \\
\text { cooling }\end{array}$ & $\begin{array}{c}\text { Standard } \\
\text { Error }\end{array}$ & $T_{m}$ & $\mathbf{T}_{\text {hysteresis }}$ \\
\hline \multirow{5}{*}{$\begin{array}{c}\text { Ficoll70 } \\
20 w t \%\end{array}$} & $D A P$ & 25.22 & 0.19 & 25.53 & 0.12 & 25.38 & -0.31 \\
\hline & $P D G F-A$ & 26.03 & 0.18 & 25.02 & 0.13 & 25.53 & 1.01 \\
\hline & KRAS & 24.41 & 0.31 & 19.23 & 0.11 & 21.82 & 5.19 \\
\hline & $H I F-1 \alpha$ & 22.57 & 0.19 & 22.44 & 0.20 & 22.51 & 0.13 \\
\hline & BCL2 & 15.01 & 0.16 & 13.62 & 0.22 & 14.32 & 1.39 \\
\hline \multirow{5}{*}{$\begin{array}{c}\text { Ficoll70 } \\
40 \text { wt } \%\end{array}$} & $D A P$ & 28.16 & 0.11 & 28.14 & 0.13 & 28.15 & 0.02 \\
\hline & $P D G F-A$ & 29.12 & 0.12 & 28.57 & 0.17 & 28.84 & 0.56 \\
\hline & KRAS & 26.08 & 0.38 & 23.33 & 0.23 & 24.70 & 2.75 \\
\hline & $H I F-1 \alpha$ & 24.36 & 0.31 & 25.31 & 0.33 & 24.83 & -0.94 \\
\hline & $B C L 2$ & 17.90 & 0.14 & 17.25 & 0.22 & 17.58 & 0.65 \\
\hline \multirow{5}{*}{$\begin{array}{c}\text { PEG200 } \\
20 w t \%\end{array}$} & $D A P$ & 34.43 & 0.13 & 34.26 & 0.24 & 34.35 & 0.17 \\
\hline & $P D G F-A$ & 34.07 & 0.12 & 34.34 & 0.15 & 34.21 & -0.26 \\
\hline & KRAS & 33.02 & 0.26 & 26.74 & 0.30 & 29.88 & 6.28 \\
\hline & $H I F-1 \alpha$ & 30.92 & 0.10 & 29.15 & 0.31 & 30.04 & 1.76 \\
\hline & $B C L 2$ & 24.03 & 0.11 & 21.90 & 0.16 & 22.96 & 2.13 \\
\hline \multirow{5}{*}{$\begin{array}{c}\text { PEG200 } \\
40 w t \%\end{array}$} & $D A P$ & 37.80 & 0.47 & 39.51 & 0.38 & 38.65 & -1.71 \\
\hline & $P D G F-A$ & 39.98 & 0.61 & 42.09 & 1.32 & 41.03 & -2.11 \\
\hline & KRAS & 35.20 & 0.51 & 32.87 & 0.46 & 34.04 & 2.33 \\
\hline & $H I F-1 \alpha$ & 34.31 & 0.20 & 35.27 & 0.27 & 34.79 & -0.97 \\
\hline & $B C L 2$ & 30.13 & 0.13 & 28.92 & 0.21 & 29.52 & 1.21 \\
\hline
\end{tabular}

$T_{m}=\left(T_{1 / 2, \text { heating }}+T_{1 / 2, \text { cooling }}\right) / 2$;

$\mathrm{T}_{\text {hysteresis }}=T_{1 / 2 \text {, heating }}-T_{1 / 2 \text {, cooling. }}$. 
Table S8 Thermodynamics without or with crowding agents.

\begin{tabular}{|c|c|c|c|c|c|c|c|c|c|}
\hline \multirow{2}{*}{$\begin{array}{l}\text { Crowding } \\
\text { agent }\end{array}$} & \multirow{2}{*}{ Promoter } & $\Delta S^{\circ}$ & SE & $\Delta H^{\circ}$ & SE & $\Delta G^{\circ}\left(20^{\circ} \mathrm{C}\right)$ & SE & $\Delta G^{\circ}\left(37^{\circ} \mathrm{C}\right)$ & \multirow[t]{2}{*}{ SE } \\
\hline & & \multicolumn{2}{|c|}{$\mathrm{kJ} \cdot \mathrm{K}^{-1} \cdot \mathrm{mol}^{-1}$} & \multicolumn{2}{|c|}{$\mathrm{kJ} \cdot \mathrm{mol}^{-1}$} & \multicolumn{3}{|c|}{$\mathrm{kJ} \cdot \mathrm{mol}^{-1}$} & \\
\hline \multirow{5}{*}{$\begin{array}{c}\text { Without } \\
\text { crowding agent } \\
(0 \%)\end{array}$} & $D A P$ & -1.11 & 0.05 & -333.62 & -16.29 & -7.11 & 0.40 & 11.82 & 0.41 \\
\hline & $P D G F-A$ & -1.10 & 0.06 & -327.52 & -18.37 & -6.13 & 0.46 & 12.51 & 0.47 \\
\hline & KRAS & -0.76 & 0.03 & -222.13 & -8.53 & -0.72 & 0.21 & 12.12 & 0.22 \\
\hline & $H I F-1 \alpha$ & -1.14 & 0.08 & -337.98 & -22.15 & -2.98 & 0.55 & 16.45 & 0.57 \\
\hline & $B C L 2$ & -0.87 & 0.02 & -249.30 & -6.32 & 5.53 & 0.16 & 20.31 & 0.16 \\
\hline \multirow{5}{*}{$\begin{array}{c}\text { Ficoll70 } \\
20 w t \%\end{array}$} & $D A P$ & -0.86 & 0.06 & -256.59 & -17.35 & -4.68 & 0.43 & 9.93 & 0.44 \\
\hline & $P D G F-A$ & -0.89 & 0.07 & -264.81 & -20.14 & -4.75 & 0.50 & 10.33 & 0.51 \\
\hline & KRAS & -0.70 & 0.04 & -205.84 & -12.15 & -0.94 & 0.30 & 10.94 & 0.31 \\
\hline & $H I F-1 \alpha$ & -0.75 & 0.02 & -223.28 & -6.59 & -2.06 & 0.16 & 10.77 & 0.17 \\
\hline & $B C L 2$ & -0.79 & 0.02 & -228.88 & -6.18 & 4.09 & 0.16 & 17.60 & 0.16 \\
\hline \multirow{5}{*}{$\begin{array}{c}\text { Ficoll70 } \\
40 \text { wt } \%\end{array}$} & $D A P$ & -0.98 & 0.07 & -294.07 & -20.20 & -7.95 & 0.50 & 8.64 & 0.51 \\
\hline & $P D G F-A$ & -0.99 & 0.08 & -297.98 & -23.95 & -8.72 & 0.59 & 8.05 & 0.61 \\
\hline & KRAS & -0.45 & 0.03 & -134.13 & -8.18 & -2.29 & 0.20 & 5.36 & 0.21 \\
\hline & $H I F-1 \alpha$ & -0.47 & 0.03 & -138.66 & -7.89 & -2.14 & 0.20 & 5.78 & 0.20 \\
\hline & $B C L 2$ & -0.77 & 0.03 & -223.17 & -8.01 & 1.65 & 0.20 & 14.69 & 0.21 \\
\hline \multirow{5}{*}{$\begin{array}{l}\text { PEG200 } \\
20 w t \%\end{array}$} & $D A P$ & -0.94 & 0.12 & -290.28 & -36.39 & -13.49 & 0.89 & 2.56 & 0.91 \\
\hline & $P D G F-A$ & -0.94 & 0.08 & -289.43 & -24.94 & -13.24 & 0.61 & 2.77 & 0.63 \\
\hline & KRAS & -0.58 & 0.06 & -175.37 & -17.23 & -5.92 & 0.42 & 3.91 & 0.44 \\
\hline & $H I F-1 \alpha$ & -1.02 & 0.09 & -308.90 & -27.21 & -10.58 & 0.67 & 6.72 & 0.69 \\
\hline & $B C L 2$ & -0.85 & 0.06 & -250.87 & -16.28 & -2.61 & 0.41 & 11.79 & 0.42 \\
\hline \multirow{5}{*}{$\begin{array}{c}\text { PEG200 } \\
40 w t \%\end{array}$} & $D A P$ & -0.62 & 0.06 & -192.05 & -18.36 & -11.12 & 0.45 & -0.62 & 0.46 \\
\hline & $P D G F-A$ & -0.78 & 0.08 & -242.97 & -24.54 & -15.20 & 0.59 & -1.99 & 0.61 \\
\hline & KRAS & -0.47 & 0.05 & -144.22 & -16.26 & -6.50 & 0.40 & 1.48 & 0.41 \\
\hline & $H I F-1 \alpha$ & -0.71 & 0.06 & -219.36 & -18.95 & -10.40 & 0.46 & 1.72 & 0.48 \\
\hline & $B C L 2$ & -0.91 & 0.07 & -274.61 & -21.09 & -8.65 & 0.52 & 6.78 & 0.53 \\
\hline
\end{tabular}

SE, standard error. 